\title{
Real-Time Motion of Open Quantum Systems: Structure of Entanglement, Renormalization Group, and Trajectories
}

\author{
Evgeny A. Polyakov \\ Russian Quantum Center, Skolkovo IC, Bolshoi Bulvar 30, bld 1, Moscow, Russia 121205*
}

\begin{abstract}
In this work we provide a complete description of the lifecycle of entanglement during the real-time motion of open quantum systems. The quantum environment can have arbitrary (e.g. structured) spectral density. The entanglement can be seen constructively as a Lego: its bricks are the modes of the environment. These bricks are connected to each other via operator transforms. The central result is that each infinitesimal time interval one new (incoming) mode of the environment gets coupled (entangled) to the open system, and one new (outgoing) mode gets irreversibly decoupled (disentangled from future). Moreover, each moment of time, only a few relevant modes (3 - 4 in the considered cases) are non-negligibly coupled to the future quantum motion. These relevant mode change (flow, or renormalize) with time. As a result, the temporal entanglement has the structure of a matrix-product operator. This allows us to pose a number of questions and to answer them in this work: what is the intrinsic quantum complexity of a real time motion; does this complexity saturate with time, or grows without bounds; how to do the real-time renormalization group in a justified way; how the classical Brownian stochastic trajectories emerge from the quantum evolution; how to construct the few-mode representations of non-Markovian environments. We provide illustrative simulations of the spin-boson model for various spectral densities of the environment: semicircle, subohmic, Ohmic, and superohmic.
\end{abstract}

\section{INTRODUCTION}

A major innovation brought by quantum mechanics is that a physical system can be in a superposition of its classical configurations. While the classical state is a point in phase space, the quantum state is a wave in the space of classical configurations [1]. All current experimental evidence suggests that the quantum theory works. Thereofore, the superposition principle is expected to apply at all scales: from a single microscopic degree of freedom to one mole of any substance with $N_{A} \propto 10^{23}$ degrees of freedom. However if we try to imagine the quantum state as a wave in the $10^{23}$-dimensional space, then the quantum reality appears before us as an object of tremendous complexity. This complexity is reflected in the notion of a Hilbert space [2].

We believe it is obvious that the overwhelming part of this potential complexity is not realized in the natural observable processes [3-5]. It is enough to review those tremendous difficulties that the experimenters face in their attemps to build a quantum computer $[6,7]$. This convinces us that there are rather effective mechanisms of how the quantum complexity decays during the natural processes.

We can put it differently: it is expected that only a tiny fraction of the Hilbert space is relevant for the observable motion of quantum systems. Therefore, in order to reduce the complexity, we need some tools to identify this relevant subspace. We can call this the problem of efficient Hilbert space decimation. The motivation is twofold: conceptual and pragmatic. Conceptually, we want to understand the stucture of quantum states and their

\footnotetext{
* evgenii.poliakoff@gmail.com
}

evolution; we want to answer the basic questions like the characterization of the boundary between the quantum and the classical reality. Pragmatically, we want to be able to calculate any property we are interested in.

The main idea of this work is that the structure of entanglement can provide answers to all these questions. The whole point is to look at the enanglement constructively. We consider it like a Lego. The bricks of this Lego are the degrees of freedom (the modes) of the environment in some trivial (separable) state. The entanglement is built by connecting these bricks via some (non) unitary operators. If we know how the entanglement is built in real time from its bricks, we can keep track of them and rigorously consider such questions as: what is the quantum complexity of real-time motion; how this complexity behaves in time: whether it saturates or grows; how to construct a few-mode approximation of the nonMarkovian environment. What is even more important, if we know how the entanglement is built, then we know how to compute the properties of quantum state by disassembling the entanglement brick-by-brick. This is the renormalization group [8-13] in one of its most advanced forms.

As a specific example, we consider in this work the model of a finite quantum system coupled to an infinite environment. This model plays important role in quantum physics. Its practical significance is due to the large number of covered situations: behavior of mesoscopic degrees of freedom in physical chemistry and condensed matter [14]; models of quantum measurement and control [15-20]. At the same time, the unique combination of simplicity and capability to exhibit many-body physics [21-24] makes it a paradigmatic model which is used to challenge and improve our perspective on quantum phenomena.

In this work we use the model of open quantum system 
to study the lifecycle of entanglement during the realtime evolution [25]. Suppose we couple the open system to the environment. Then it starts to emit quanta into the environment. Some of them will be reabsorbed, but a significant number of quanta will fly further and further into the environment, therefore entangling increasingly more modes of the environment. This leads to a combinatorial growth of the dimension of the joint wavefunction. Such a combinatorial growth of complexity is called the entanglement barrier [26] and it presents a significant obstactle both to interpretation and real-time computational methods.

In an attempt to make sense of the entanglement barrier let us ask the question: what is the ultimate fate of the emitted quantum field? First of all, we expect that the emitted field becomes asymptotically decoupled from the future motion of open system. For concreteness, let us assume that at some time moment $t^{*}$ a certain mode $\phi_{\text {out }}$ of the environment becomes effectively and irreversibly decoupled. Then it is important how this mode is entangled to the rest of the environment. The mode $\phi_{\text {out }}$ can be entangled to those modes of the environment which were coupled to open system before $t^{*}$. We say figuratively that $\phi_{\text {out }}$ is entangled to the past. However, $\phi_{\text {out }}$ can not be significantly entangled to any mode $\phi_{\text {in }}$ which couples to open system after $t^{*}$. This is because the entanglement can be only created via unitary evolution under coupling, which is effectively absent for $\phi_{\text {out }}$ after $t^{*}$. Therefore, we say figuratively that $\phi_{\text {out }}$ is not entangled to the future. Now suppose that we have succeed to arrange the following two streams of modes: (i) the stream of incoming modes such that the mode $\phi_{\text {in }}(t)$ assigned to the time moment $t$ couples to open system only after $t$; (ii) the stream of outgoing modes such that the mode $\phi_{\text {out }}(t)$ assigned to the time moment $t$ is irreversibly decoupled from the future motion after $t$. This system of modes leads to the entanglement structure depicted in Fig. 1.

From this picture one sees that our entanglement Lego contains the two types of bricks: the incoming modes $\phi_{\text {in }}\left(t_{p}\right)$ and the outgoing modes $\phi_{\text {out }}\left(t_{p}\right)$. If we look at how the entanglement builds forward in time, we can observe that these two types of bricks play different roles. The incoming modes $\phi_{\text {in }}\left(t_{p}\right)$ are sequentially attached to the structure via the evolutions $\widehat{U}_{p}^{\prime}$ thus increasing its complexity. At the same time, the outgoing modes $\phi_{\text {out }}\left(t_{p}\right)$ do not participate in the construction. Therefore, we can remove them by tracing out them as soon as they emerge. This is how we can disassemble the entanglement by continuously removing all the emerging such "idle" bricks. The competition between the two processes, the attachment of $\phi_{\text {in }}\left(t_{p}\right)$ and the removal of $\phi_{\text {out }}\left(t_{p}\right)$, determines the final complexity of the structure. In Fig. 1 we have assumed that $\phi_{\text {in }}\left(t_{p}\right)$ and $\phi_{\text {out }}\left(t_{p}\right)$ occur with equal rates in time. Then, each moment of time only a finite number of bricks (in Fig. 1 they are the vertical salmon-colored lines connecting the adjacent evolutions) are attached to the structure. The quantum

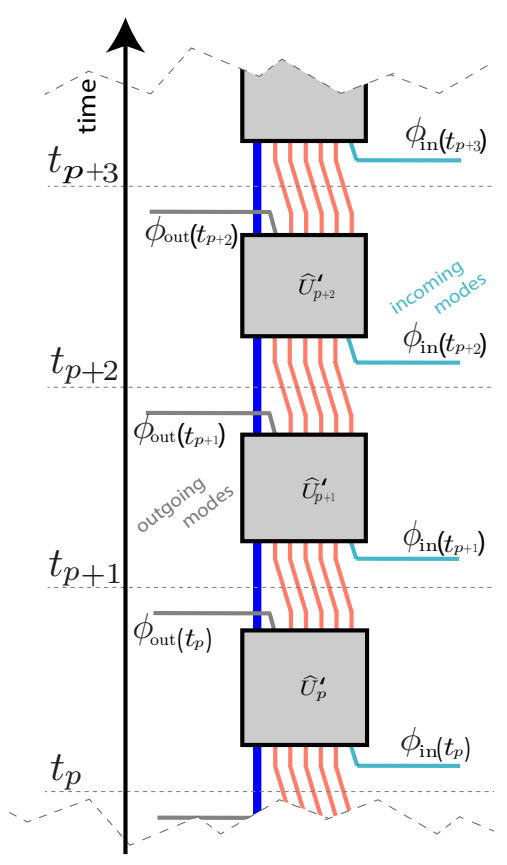

Figure 1. The temporal structure of entanglement which follows from the existence of the streams of incoming and outgoing modes. The blue line depicts the quantum numbers of the open system. The evolution $\widehat{U}_{p}^{\prime}$, which occurs during each infinitesimal time interval $\left[t_{p}, t_{p+1}\right]$, couples one additional incoming mode $\phi_{\text {in }}\left(t_{p}\right)$. The corresponding quantum numbers are denoted by the turquoise line. After that, one additional ougoing mode $\phi_{\text {out }}\left(t_{p+1}\right)$ becomes irreversibly decoupled from the future evolution. Its quantum numbers are denoted by the gray line.

complexity of real-time motion only survives in these bricks. The quantum complexity will saturate and become bounded with time if the occupation of the coupled modes is bounded. In other words, the flux $j_{\text {in }}\left(t_{p}\right)$ of the quanta emitted by the open system should balance the flux $j_{\text {out }}\left(t_{p}\right)$ of the quanta leaking out to the outgoing modes.

The structure in Fig. 1 yields a few-mode representation of the bath. If we consider the reduced joint state of open system and of the coupled modes, then all the effects of the environment on the system will be taken into account. In this work we verify this idea by considering the model of open quantum system in an environment with subohmic, Ohmic, superohmic, and semicircle (waveguide) spectral density.

The structure in Fig. 1 indicates that the time plays the role of a flow parameter of some renormalization group: the movement in time is accompanied with the emergence of degrees of freedom which are unentangled to the future flow. In the language of RG they are called the irrelevant degrees of freedom. Such degrees of freedom are iteratively traced out while computing the flow. Here we mean the modern varinants of RG, like the entanglement renormalization (ER) [8-11]. In this work we 
propose a numerical implementation of the real-time $\mathrm{RG}$ which follows from the Fig. 1. We verify the idea by a calculation for the environment with a semicircle spectral density.

The second thing we expect to eventually happen is that the emitted field becomes observed. For example, one can imagine that the environment is filled with observers at an exteremy low density. So that when the emitted field hits the observer, it has already effectively decoupled from the open system. These observers can percieve e.g. the signal of displaced vacuum of the environment (i.e. they observe some global classical background of the environment). Such a signal is expected to be a superposition of the stochastic vacuum noise and some deterministic motion due to interaction with the open system [27]. Because of the entanglement structure in Fig. 1, such a measurement does not disturbs the future quantum evolution: the trace over the outgoing modes can be represented as a stochastic average over the ensemble of their measurement signals. In Fig. 1 this corresponds to replacing each occuring gray line with some classical noise. Then we immediately conclude that there exist two kinds of entanglement: (i) the entanglement which is coupled to the future quantum motion. It appears to be a genuinely quantum phenomenon. (ii) The outgoing entanglement which is irreversibly decoupled from the future quantum motion (and which is carried by the outgoing modes $\phi_{\text {out }}\left(t_{p}\right)$ ). The latter kind of entanglement is equivalent to the stochastic ensemble of classical signals. This fact has both pragmatic and conceptual consequences. Pragmatically, we can efficiently simulate/analyze the outgoing entanglement via the Monte Carlo sampling of the signal realizations. The resulting procedure, the renormalization group for quantum trajectories, is presented in this work. Conceptually, we conjecture that the outgoing entanglement is the carrier of classical reality. We verify the idea by a calculation for the environment with a subohmic spectral density.

In the following sections we develop this picture up to numerical schemes by proposing a measure of average coupling to the future.

This work is structured as follows. We place our ideas into the current research context in sec. II. The model of open quantum system and its environment are introduced in sec. III. Then in sec. IV we study how the emitted field decouples from its source. We encourage the reader to pay special attention to the Figs. 4 and 11 which describe the physical mechanism of how the stream of the incoming modes and of the irreversibly decoupled (outgoing) modes emerge in real time. We verify our ideas by providing the calculations for the subohmic/Ohmic/superohmic and semicircle environments. The reader who is interested in the justification of the real-time RG should pay attention to sec. IV C and to the discussuon of Figs. 8 and 9. The Schrodinger equation for the model of open quantum system is reformulated in terms of the incoming and outgoing modes in sec. V. As a result, in Fig. 17 we obtain the entangle- ment structure of Fig. 1. In sec. VI the outgoing modes are traced out in real time as soon as they occur: this is the renormalization group for density matrix. We verify this idea by a calculation for the semicircle spectral density (waveguide environment). In sec. VIF, Fig. 25, we present a calculation which supports our conjecture that there is a balance between the fluxes of incoming and outgoing quanta, i.e. the complexity of real-time motion is expected to saturate. In section VII we consider the Markovian limit of our RG procedure. In sec. VIII we implement another alternative for the RG procedure: to measure the irrelevant modes in real time as soon as they occur. We verify this by a calculation for the environemnt with a subohmic spectral density. Our approach is compared with a state-of-the-art tensor network method (TEMO [28]). In section IX we introduce the measure of temporal entanglement. We conclude in sec. X. There are also four appendices where we provide some implementation details of our RG procedures.

\section{RELATION TO OTHER APPROACHES}

Ultimately our approach is to find such a quantum circuit (Fig. 1) which shows how the entanglement is built along the flow (which is the real time in our case) from some set of uncoupled degrees of freedom. This promotes the viewpoint of entanglement as some kind of Lego. Its bricks are the uncoupled degrees of freedom. These bricks are sticked together by applying (non)unitary operations. The merit of this viewpoint is that the observable properties of the resulting complex many-body states can be computed by gradually disassembling this Lego brick-bybrick.

Actually this viewpoint is close in spirit to the ideas of the entanglement renormalization (ER) [8-11]. However, we extend ER in the following two aspects.

First, ER studies how the entanglement is arranged over the increasing length scales. In other words, for ER the relevant degrees of freedom are those which contribute to the low-energy ('infrared') properties. While this is appropriate for the description of low-temperature equilibrium properties, it becomes unjustified in the case of real-time evolution. During the real-time motion, the widely-separated energy scales may become coupled e.g. after a sudden quench of parameters. We avoid this problem since in our approach the relevant degrees of freedom are exactly those which have a non-negligible coupling to the future evolution.

The second difference is how our circuit model is derived. Below we introduce the metric $I_{2}[\phi ; \tau]$ which measures how much the given mode $\phi$ at a given time moment $\tau$ is coupled to the future motion. This way we rigorously identify the modes $\phi_{\text {out }}$ with are irreversibly irrelevant (decoupled) because their metric $I_{2}\left[\phi_{\text {out }} ; \tau\right]$ is below a certain treshold. Thefore, we prove our entanglement model. This should be contrasted to the reasoning behind ER which is more heuristic, e.g. by matching the 
structure of a tensor network against the expected entanglement scaling laws [29]. Main justification of ER comes from the corpus of the numerical calculation results.

In literature there are many other formal ways to describe the evolution of open systems. They can be divided into the following four groups. The first group are the methods which do not rely on the structure of entanglement. They are the master equations [14, 30] and their stochastic unravelings [31-34], path integrals with influence functionals [35-40], equivalent chain representations [41], pseudomodes [42, 43] and Markovian embeddings [44].

The second group of methods consists of the straightforward extensions of the Wilson renormalization group ideas (e.g. the logarithmic discretization of energy scales, the focus on the infrared limit) to the real time case: impurity numerical renormalization group [24]. These methods make the (often unjustified) assumption that the real-time structure of entanglement is similar to the equilibirium case.

The third group of methods rely on the tensor network models of quantum states and evolution [28, 45-47], usually within the framework of the influence functional formalism. The language of tensor networks is suitable for the description of the structure of entanglement [48]. However, in these methods the main focus is to employ the linear algebra methods (e.g. SVD) to compress the numerical representation of quantum states. They neither reveal nor exploit the lifecycle of entanglement in real time.

The final group consists of methods which are derived from different principles, but which, in our opinion, turn out to exploit implicitly some aspects of the structure described in Fig. 1. These are the non-Markovian quantum state diffusion $[27,49,50]$ and stochastic Schrodinger equations [51-54]; various variants of the approach of hierarchical equations of motion [55-61].

\section{MODEL OF OPEN QUANTUM SYSTEM}

Here we describe the model for which we implement our ideas in this paper: the open quantum system. The latter is defined by a Hamiltonian $\widehat{H}_{\mathrm{s}}$. The system is coupled to bosonic environment via some operator $\widehat{s}$. The environment is harmonic with a Hamiltonian $\widehat{H}_{\mathrm{b}}$,

$$
\widehat{H}_{\mathrm{b}}=\int_{0}^{+\infty} d \omega \widehat{a}^{\dagger}(\omega) \widehat{a}(\omega) .
$$

Here the environment's degrees of freedom are $\left[\widehat{a}(\omega), \widehat{a}^{\dagger}\left(\omega^{\prime}\right)\right]=\delta\left(\omega-\omega^{\prime}\right)$. The environment is coupled to the open system via some site operator

$$
\widehat{b}=\int_{0}^{+\infty} d \omega c(\omega) \widehat{a}(\omega)
$$

The coupling constant is related to the spectral density $J(\omega)=\pi|c(\omega)|^{2}$. The total Hamiltonian of the joint system is

$$
\widehat{H}_{\mathrm{sb}}=\widehat{H}_{\mathrm{s}}+\widehat{s}^{\dagger} \widehat{b}+\widehat{s}^{\dagger}+\widehat{H}_{\mathrm{b}}
$$

In the interaction picture wrt the free environment we have

$$
\widehat{H}_{\mathrm{sb}}(t)=\widehat{H}_{\mathrm{s}}+\widehat{s}^{\dagger}(t)+\widehat{s}^{\dagger} \widehat{b}(t)
$$

where

$$
\widehat{b}(t)=\int_{0}^{+\infty} d \omega c(\omega) \widehat{a}(\omega) e^{-i \omega t}
$$

For convenience we assume that initially at $t=0$ the environment is in its vacuum state $|0\rangle_{\mathrm{b}}$, so that the initial joint state is

$$
|\Phi(0)\rangle_{\mathrm{sb}}=\left|\phi_{0}\right\rangle_{\mathrm{s}} \otimes|0\rangle_{\mathrm{b}}
$$

where $\left|\phi_{0}\right\rangle_{\mathrm{s}}$ is the initial state of the system. The vacuum initial condition can be easily generalized to a finite temperature state of the environment [27, 62]. Here the subscripts 'b', 's', and 'sb' designate the belonging to the Hilbert space of the open system $\mathcal{H}_{\mathrm{s}}$, the Fock space $\mathcal{F}_{\mathrm{b}}$ of environment, and the joint space $\mathcal{H}_{\mathrm{s}} \otimes \mathcal{F}_{\mathrm{b}}$ of the open system and environment correspondingly.

\section{HOW THE QUANTUM FIELD GRADUALLY DECOUPLES FROM ITS SOURCE}

In this sections we find what are the incoming and outoing modes from Fig. 1. The entanglement is generated by coupling. Therefore, the analysis of the entanglement structure is essentially the analysis of the decoupling mechanism. In this section we consider the superspositions of single-quantum states which are emitted by the open system, and describe the mechanism behind their irreversible decoupling which was proposed in $[27,63]$. Later in the next section we apply this mechanism to the full many-quanta case.

\section{A. Physical mechanism of decoupling}

During its evolution the open system emits and absorbs quanta in the environment. There are several important asymmetries between the emission and absorption processes which we discuss and exploit here.

\section{Parametrization of the emitted quantum field}

The Fock space of the environment (1) is infinite. The important nontrivial fact is that the Fock space of the 


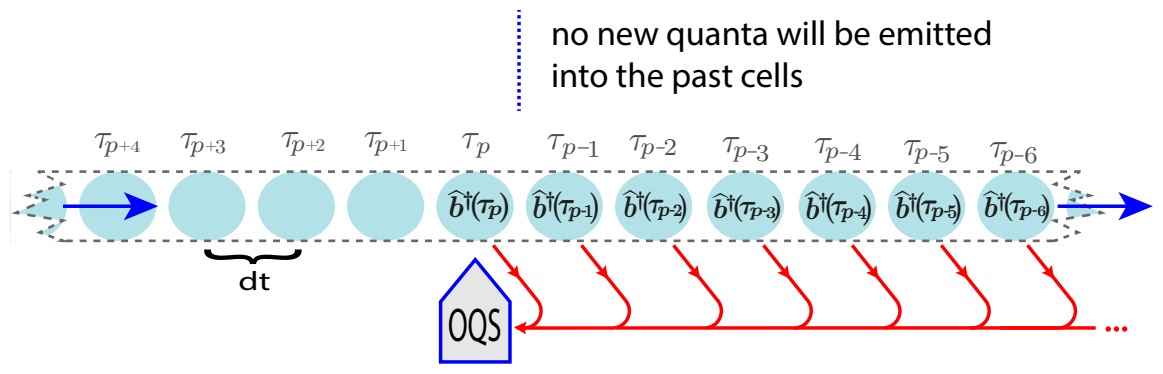

Figure 2. We may think of time as a stream of infinitesimally close time moments $\tau_{0}, \tau_{1}, \ldots, \tau_{p}, \tau_{p+1}, \ldots$ The time moves with constant speed in one direction (the blue arrows) like a tape inside a tape recorder (or a Turing machine). Each time moment $\tau_{p}$ corresponds to a cell of the tape (the light turquoise circles). Then the open system acts like a write/erase head. At the time moment $\tau_{p}$, the head is located in front of the corresponding cell. The head writes new quanta strictly to the cell $\tau_{p}$ (which is denoted by $\left.\widehat{b}^{\dagger}\left(\tau_{p}\right)\right)$ : it is a local operation. At the same time, the erasing of quanta is a causal but long-range operation: the quanta can be erased from an arbitrarily distant past cell $\tau_{r}$, with a slowly decaying amplitude $M\left(\tau_{p}-\tau_{q}\right)$ (red arrows). Erasing is the only way for the past cells to interact with the future motion. What is important is that in this representation the compexity of the emitted quantum field grows gradually. For example, after one infinitesimal time step the Fock space of the emitted field is spanned by only one mode $\widehat{b}^{\dagger}\left(\tau_{0}\right)$.

quantum field which can be emitted by open system is dramatically smaller. Indeed, since the open system sits at one local site $\widehat{b}^{\dagger}$ of the environment, clearly it cannot resolve the full Fock space of the environment. The spatiotemporal resolution of the emitted field is determined by the spectral properties of $\widehat{b}^{\dagger}(t)$.

Formally, from the Hamiltonian (4) it is seen that at a time moment $t$ the quanta are emitted via $\widehat{b}^{\dagger}(t)$. Let us assume that the evolution happens during the time interval $[0, t]$, starting from the initial state $|\Phi(0)\rangle_{\mathrm{sb}}$, eq. (6). One can imagine that this time interval consists of a sequence of $N$ time moments $\tau_{0}, \tau_{1} \ldots, \tau_{p}, \tau_{p+1}, \ldots$, $0 \leq \tau_{p} \leq t, p=0 \ldots N$, which are equally spaced at an infinitesimal interval $d t$. Then at each time moment $\tau_{p}$ the new quanta are created via $\widehat{b}^{\dagger}\left(\tau_{p}\right)$. This means that the wavefucntion $|\Phi(t)\rangle_{\mathrm{sb}}$ can only depend on $\widehat{b}^{\dagger}\left(\tau_{0}\right), \ldots \widehat{b}^{\dagger}\left(\tau_{p}\right), \ldots \hat{b}^{\dagger}\left(\tau_{N}\right)$. We may write it in the following form

$$
\begin{aligned}
& |\Phi(t)\rangle_{\mathrm{sb}}=\sum_{n_{0}=0}^{\infty} \ldots \sum_{n_{N}=0}^{\infty} \frac{1}{\sqrt{n_{0} ! \ldots n_{N} !}} \\
& \quad \times\left|\phi\left(n_{0} \ldots n_{N}\right)\right\rangle_{\mathrm{s}} \otimes \widehat{b}^{\dagger n_{0}}\left(\tau_{0}\right) \ldots \widehat{b}^{\dagger n_{N}}\left(\tau_{N}\right)|0\rangle_{\mathrm{b}} .
\end{aligned}
$$

We can visualize the resulting evolution by imagining it as a tape recorder, Fig. 2: the time is like a tape which moves with a constant speed [64]. This tape is divided into discrete cells, one cell for each time moment $\tau_{p}$. Then the open system is like a read/erase head. At the time moment $\tau_{p}$ the head writes new quanta into the $\tau_{p}$ th cell of the tape. At the same time, the head can erase quanta from any past cell $\tau_{q}, \tau_{q} \leq \tau_{p}$, with the amplitude

$$
\begin{array}{r}
{\left[\widehat{b}\left(\tau_{p}\right), \widehat{b}^{\dagger}\left(\tau_{q}\right)\right]=\frac{1}{\pi} \int_{0}^{+\infty} d \omega J(\omega) e^{-i \omega\left(\tau_{p}-\tau_{q}\right)}} \\
=M\left(\tau_{p}-\tau_{q}\right),
\end{array}
$$

where $M\left(\tau_{p}-\tau_{q}\right)$ is the so-called memory function of the environment. This equation follows from eq. (5) and from the commutation relation $\left[\widehat{a}(\omega), \widehat{a}^{\dagger}\left(\omega^{\prime}\right)\right]=$ $\delta\left(\omega-\omega^{\prime}\right)$. The past cells may be entangled with each other and with the head (open system), as it follows from (7).

We see that in this picture both the creation and the absorption of quanta are causal processes: the future cells with $\tau_{q}>\tau_{p}$ are empty and uncoupled from the open system. They couple to the evolution one-by-one as the tape moves in front of the head. We call them the stream of incoming modes. They are the bricks of the Lego from which the entanglement is being built in real time.

The first important asymmetry is that the new quanta can be written in the present and future cells, with $\tau_{q} \geq$ $\tau_{p}$, whereas no new quanta are created in the past cells with $\tau_{q}<\tau_{p}$. The past cells are coupled to the future motion only via the annihilation of quanta.

The second important asymmetry is that the creation of new quanta is a local operation, whereas the annihilation of quanta is a long-range operation, since the memory function $M(t)$ always has the inverse-power law tails $[27,63]$. Indeed, since the physical spectral function $J(\omega)$ cannot have the negative-frequency components, it should go to zero as a certain power of $\omega: \omega^{p_{0}} \theta(\omega)$, where $\theta$ is a Heaviside function, and $p_{0}>0$. This leads to a tail $\propto( \pm i t)^{-p_{0}-1}$ in the large- $t$ asymptotic behaviour of $M(t)$. Moreover, every frequency $\omega_{k}$, where $J\left(\omega_{k}+\delta \omega\right)$ has a discontinous part $\delta \omega^{p_{k}} \theta( \pm \delta \omega)$, leads to an addi- 
tional tail $\propto e^{-i \omega_{k} t}( \pm i t)^{-p_{k}-1}$ in $M(t)$. These discontinuities can be e.g. the band edges. Such tails were called the memory channels in $[27,63]$.

For illustration, let us consider the widely used model of the environment with a spectral density

$$
J(\omega)=\frac{\alpha \omega_{\mathrm{c}}}{2}\left[\frac{\omega}{\omega_{\mathrm{c}}}\right]^{s} \exp \left(-\frac{\omega}{\omega_{\mathrm{c}}}\right)
$$

It covers many types of environment by varying the parameter $s$ : the subohmic $(0 \leq s<1)$, Ohmic $(s=1)$ and superohmic $(s>1)$. This spectral density corresponds to the memory function

$$
M(t)=\frac{\alpha \omega_{\mathrm{c}}^{2}}{2 \pi} \frac{\Gamma(s+1)}{\left(1+i t \omega_{\mathrm{c}}\right)^{s+1}},
$$

which has a tail $\propto \tau^{-s-1}$ due to the discontinuity at the frequency $\omega=0$. Another example is the waveguide environment (in the Schrodinger picture)

$$
\widehat{H}_{\mathrm{b}}=\sum_{j=1}^{\infty}\left\{h \widehat{a}_{j+1}^{\dagger} \widehat{a}_{j}+h \widehat{a}_{j}^{\dagger} \widehat{a}_{j+1}+\varepsilon \widehat{a}_{j}^{\dagger} \widehat{a}_{j}\right\},
$$

with the open system being coupled to the first site $\widehat{a}_{1}$. In the interaction pictire with respect to $\widehat{H}_{\mathrm{b}}$, we get $\widehat{b}(t) \equiv \widehat{a}_{1}(t)=\exp \left(i t \widehat{H}_{\mathrm{b}}\right) \widehat{a}_{1} \exp \left(-i t \widehat{H}_{\mathrm{b}}\right)$. Such an environment has a finite band of energies $[\varepsilon-2 h, \varepsilon+2 h]$, and the memory function is

$$
M(t)=e^{-i \varepsilon t} \frac{J_{1}(2 h t)}{h t} \propto t^{-\frac{3}{2}} e^{-i(\varepsilon-2 h) t}+t^{-\frac{3}{2}} e^{-i(\varepsilon+2 h) t},
$$

where now we have two tails, one tail per band edge at the frequencies $\omega_{1}=\varepsilon-2 h$ and $\omega_{2}=\varepsilon+2 h$.

This long-range character of $M(t)$ is a problem because it leads to a large number of past cells which are entangled and significantly coupled to the future motion. As a result, the complexity of real-time motion accumulates combinatorially-fast and becomes prohibitive.

In order to solve this problem, let us cosider the amplitude of the annihilation process. Suppose that by the time moment $\tau_{p}$ one quantum was emitted. Its state $|\phi\rangle_{\mathrm{b}}$ is a superposition of the cell-write events:

$$
|\phi\rangle_{\mathrm{b}}=\left\{\phi_{0} \widehat{b}^{\dagger}\left(\tau_{0}\right)+\ldots+\phi_{p-1} \widehat{b}^{\dagger}\left(\tau_{p-1}\right)\right\}|0\rangle_{\mathrm{b}} .
$$

Here for the moment we neglect the degrees of freedom of the open system (in the next section $\mathrm{V}$ we derive the full equations of motion). Now suppose that in the future the open system points to some cell $\tau_{p^{\prime}}$, with $\tau_{p^{\prime}}>\tau_{p}$. Then it will erase this quantum state (13) with the amplitude

$$
\mathcal{A}_{p^{\prime} p}[\phi]=\sum_{r=0}^{p-1} M\left(\left(p^{\prime}-r\right) d t\right) \phi_{r} .
$$

We see that the quanta in the past cells are coupled to the future via the convolution with $M(t)$. Now we recall that

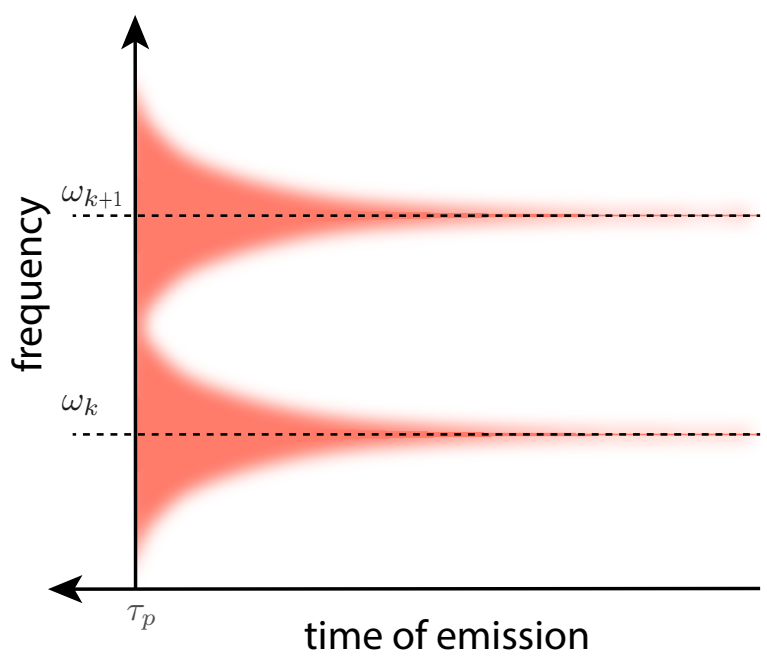

Figure 3. The emitted quantum field is coupled to the future motion only via convolution with the memory function, eq. (14). As a result, the spectral content of the emitted quanta is completely decoupled from the future motion, except a progressively small vicinity (salmon-colored area) of the singular frequencies $\omega_{k}, \omega_{k+1}, \ldots$ where the spectral function $J(\omega)$ has discontinuities. These singular frequencies may be e.g. the boundaries of energy bands or other sharp features of the spectral density.

for the distant past the memory function $M(t)$ behaves as a superposition of its tails $M_{k}(t)=e^{-i \omega_{k} t}( \pm i t)^{-p_{k}-1}$. Every such tail has the property that its local spectrum gradually becomes more and more narrowed to the frequency $\omega_{k}$ as the time argument $t$ is increased. Equivalently, one may say that as $t$ is increased, there is an increasingly large time scale $\Delta(t)$ over which the tail $M_{k}(t)$ can be considered as being effectively constant. Therefore, starting from $t$ every spectral component of $\phi$ outside the frequency range $\approx\left[\omega_{k}-\Delta(t)^{-1}, \omega_{k}+\Delta(t)^{-1}\right]$ will be averaged to zero by convolving with $M(t)$ in eq. (14). Physically this means that in the remote past, the spectral content of the emitted quanta is completely decoupled from the future motion, except a progressively small vicinity of these singular frequencies $\omega_{k}$, see Fig. 3.

The analysiz above convinces us that as the time proceeds, new modes of the environment should continously emerge which are effectively decoupled from the future motion, see Fig. 4. We call these the stream of outgoing modes. We will construct them formally in the following section.

Now suppose we succeeded in constructing the degrees of freedom which represent the different spectral areas in Fig. 4. Namely, the cells from Fig. 2 are treated as independent quantum degrees of freedom. Then the frame is changed (via a unitary transform, Fig. 5) so that instead of the past cells we have: (i) the cells which are significantly coupled to the future (the relevant modes, from the salmon-colored area in Fig. 4) and (ii) the cells 


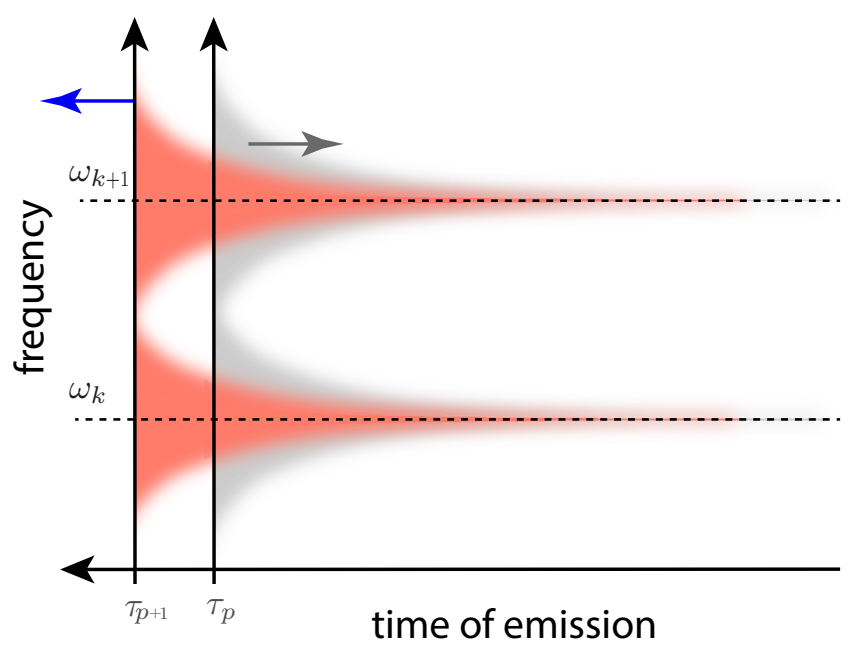

Figure 4. When the real-time evolution is propagated forward one time step (blue arrow), from $\tau_{p}$ to $\tau_{p+1}$, the emitted quantum field is shifted one step further into the past (gray arrow). Then the frequency scales $\Delta(t)^{-1}$ slightly shrink. As a result, each propagation step yields new spectral content (gray area) which is irreversibly decoupled from the future motion. The gray area should correspond to some continuously emerging outgoing modes of the environment.

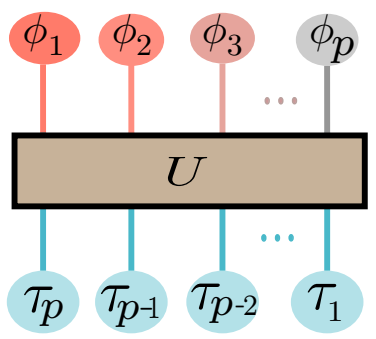

Figure 5. If we find the degrees of freedom (the modes) which correspond to the coupled and decoupled spectral components of the emitted quantum field, then we can switch to the basis $\phi_{1}, \phi_{2}, \ldots$ of such modes by applying a suitable unitary transform $U$ to the past cell degrees $\tau_{1}, \tau_{2}, \ldots, \tau_{p}$ of freedom of the time tape. The new cells $\phi_{k}$ are numbered in the decreasing order of their significance for the future evolution. Their color match the color of the spectral areas in Fig. 4.

which are effectively and irreversibly decoupled from the future (the outgoing modes, from the gray area in Fig. 4), see Fig. 6. We expect that this way the coupling to the past will become short-ranged, and the complexity of the real-time motion will become bounded. This is because the decoupled past modes can be traced out as we demonstrate below in this work.

\section{B. How to find the outgoing modes}

In this section we implement the intuition of Fig 3: we find the modes which are significantly coupled to the future motion (lie inside the salmon-colored area) and those which are irreversibly decoupled (lie outside the samlon-colored area).

Let us again assume that by the time moment $\tau_{p}$ there is a single-quantum state of the tape (13). Recall that $\mathcal{A}_{p^{\prime} p}[\phi]$ from eq. (14) is the amplitude to annihilate this quantum at a later time moment $\tau_{p^{\prime}}$. We introduce the short-hand bra-ket notation for the equation (14):

$$
\mathcal{A}_{p^{\prime} p}[\phi]=\left(p^{\prime}|M| \phi\right),
$$

where the matrix $M$ is the discretized memory function $M_{l r}=M((l-r) d t)$, with $l, r \geq 0$. The set of amplitudes $\phi_{0}, \ldots, \phi_{p-1}$ is considered as a semiinfinite vector $\phi_{r}, r \geq$ 0 , with $\phi_{r}=0$ for $r \geq p$. This vector is denoted in the bra-ket notation as $\mid \phi)$. The notation $(p \mid \phi)$ means the amplitude on the cell $\tau_{p}:(p \mid \phi)=\phi_{p}$.

In order to estimate the significance of $\phi$ for the future quantum motion, we can evaluate the average intensity of annihilation processes over all the future time moments:

$$
I_{q}\left[\phi ; \tau_{p}\right]=\sum_{p^{\prime}=p}^{\infty}\left(\left|\left(p^{\prime}|M| \phi\right)\right|^{q}\right)^{\frac{1}{q}} /(\phi \mid \phi)^{\frac{1}{2}}
$$

with some parameter $q>0$. The quantity $I_{q}\left[\phi ; \tau_{p}\right]$ is the power mean of $\mathcal{A}_{p^{\prime} p}[\phi]$ over the time interval $\left[\tau_{p}, \infty\right)$. Here the denominator in (16) removes the trivial dependence on normalization of $\phi$. It is natural to expect that the mode $\phi_{1}$ which makes the largest average contribution to the future annihilation processes is the most important for the future motion. Such a mode can be found by maximizing $I_{q}\left[\phi ; \tau_{p}\right]$. The second most imortant mode $\phi_{2}$ can be found by maximizing $I_{q}\left[\phi ; \tau_{p}\right]$ subject to the orthogonality constraint $\left(\phi_{1} \mid \phi_{2}\right)=0$. Repeating iteratively this process by maximizing $I_{q}\left[\phi ; \tau_{p}\right]$ subject to the constraint of orthogonality to all the previously found modes, we find the fastest decoupling basis of modes $\phi_{1} \ldots \phi_{p}$.

Let us recall the intuitive picture of Fig. 3. The annihilation amplitude (15) has the convolutional form. The intensity $I_{q}\left[\phi ; \tau_{p}\right]$ is the average magnitude of such convolutions which are shifted to future times. Therefore, we expect that the modes which maximize $I_{q}\left[\phi ; \tau_{p}\right]$ correspond to the salmon-colored spectral area in Fig. 3. The modes which yield the least significant contribution to $I_{q}\left[\phi ; \tau_{p}\right]$ lie ouside the salmon-colored spectral area of Fig. 3.

In this paper we consider the case of a root mean square intensity $I_{2}\left[\phi ; \tau_{p}\right]$. In this case the intensity assumes the form

$$
I_{2}^{2}\left[\phi ; \tau_{p}\right]=(\phi|K(p)| \phi),
$$

where the matrix $K(p)$ is

$$
K(p)_{r^{\prime} r}=\sum_{p^{\prime}=p}^{\infty} M^{*}\left(\left(p^{\prime}-r^{\prime}\right) d t\right) M\left(\left(p^{\prime}-r\right) d t\right),
$$




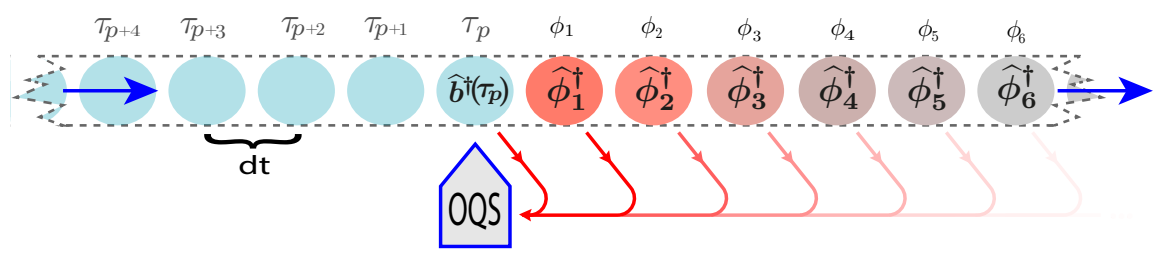

Figure 6. In the basis of the modes $\phi_{k}$ whose significance for future motion rapidly decreases, the coupling to the past is expected to become short-ranged.

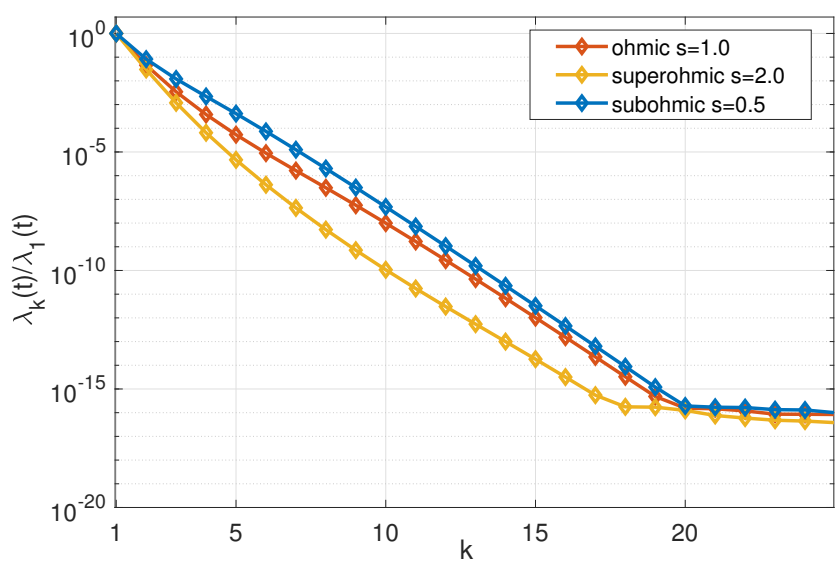

Figure 7. The average contribution of the modes $\phi_{k}$ of the fastest decoupling basis to the future evolution decays exponentially fast. Here the cases of subohmic $(s=0.5)$, ohmic $(s=1.0)$, and superhomic $(s=2.0)$ environments are considered for the time moment $t_{p}=p d t=100$ and $d t=0.01$. The saturation on the level of $10^{-16}$ is due to roundoff errors.

$0 \leq r, r^{\prime}<p$. Then the fastest decoupling basis $\phi_{1} \ldots \phi_{p}$ is given by the eigenvectors of $K(p)$,

$$
\left.\left.K(p) \mid \phi_{k}\right)=\lambda_{k} \mid \phi_{k}\right),
$$

where $k=1,2, \ldots$, and we sort the eigenvalues $\lambda_{k}(p)$ in the descending order.

\section{Example calculations: some tests of the ideas}

As illustration, we resent in Fig. 7 the plot of normalized eigenvalues $\lambda_{k}(p) / \lambda_{1}(p)$ for the cases of subohmic $(s=0.5)$, ohmic $(s=1.0)$ and superohmic $(s=2.0)$ environments, eq. (10), for the time moment $t_{p}=p d t=100$. One observes that the average coupling of these eigenvectors to the future evolution decays exponentially fast.

If the intuition of Fig 3 is valid, then the oscillations of the eigenvectors $\phi_{k}$ should rapidly slow down so that the spectral content of $\phi_{k}$ remains in the progressively small vicinity of the only singular frequency $\omega=0$. In Fig. 8 we present the plots of $\phi_{10}$ for the cases of subohmic $(s=0.5)$, ohmic $(s=1.0)$ and superohmic $(s=2.0)$ environments. It is seen that the scale of their oscillations

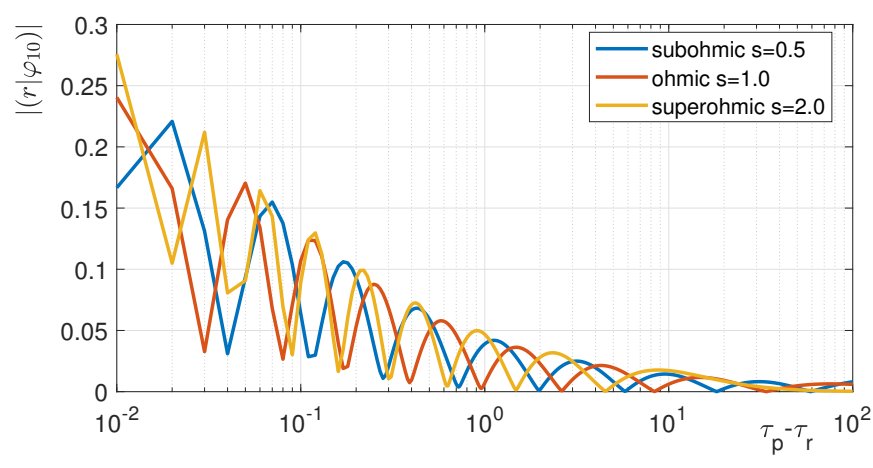

Figure 8. Plot of the fastest decoupling basis function $\left|\left(r \mid \phi_{10}\right)\right|$ vs $\tau_{p}-\tau_{r}$ for $t_{p}=p d t=100$, for the cases of subohmic $(s=0.5)$, ohmic $(s=1.0)$, and superhomic $(s=2.0)$ environments. Observe that we plot with respect to the "time of delay" $\tau_{p}-\tau_{r}$ between the present $\tau_{p}$ and the past $\tau_{r}$. The scales of their oscillations are approximately constant on the logarithmic scales of delay times.

is almost constant on the logarithmic scale of times $\tau_{r}$, which supports our intuition.

A more stringent test of our intuition is to compute the most important mode $\phi_{1}$ for the waveguide memory function (12). If the Fig. 3 is valid, and if the first eigenvalues of $K(p)$ indeed sample the salmon-colored spectral area, than the tails of $\phi_{1}$ should behave as a superposition of $e^{+i(\varepsilon-2 h) t}$ and $e^{+i(\varepsilon+2 h) t}$. In Fig. 9 we present the plot of $\phi_{1}$ for the waveguide with $\varepsilon=1$ and $h=0.05$.

Taking into account the exponentially fast decoupling of the modes $\phi_{k}$, we can keep the first $m$ modes $\phi_{1}, \ldots \phi_{m}$ as the relevant modes, and consider the rest modes $\phi_{m+1}, \phi_{m+2}, \ldots$ as the outgoing modes. The latter are irreversibly decoupled, which is supported by the following property of the average intensity:

$$
I_{q}\left[\phi ; \tau_{p^{\prime}}\right] \leq I_{q}\left[\phi ; \tau_{p}\right] \text { for } \tau_{p^{\prime}} \geq \tau_{p},
$$

which follows from its definition (16). 


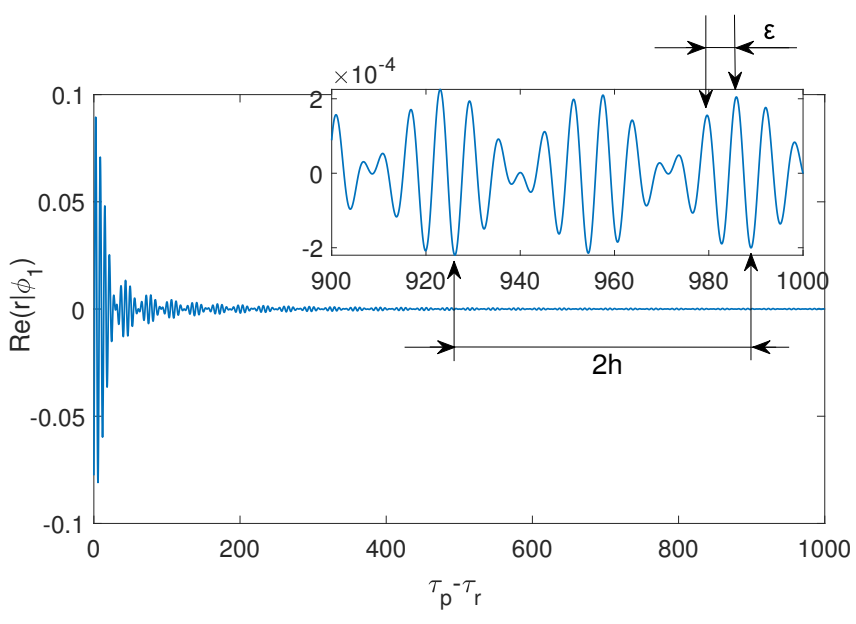

Figure 9. Plot of the most importat mode $\phi_{1}$ for the waveguide memory function eq. (12) with $\varepsilon=1$ and $h=0.05$. It is seen on the inset that the tail $\phi_{1}$ behaves like a superspostion of oscillations with the frequencies $\varepsilon-2 h$ and $\varepsilon-2 h$. This supports the intuition of Fig. 3 that the coupled spectral content of the quanta emitted in the past shrinks to the singular frequencies of the spectral function.

\section{Implications for the renormalization group: the choice of relevant space}

Looking at the fastest decoupling basis modes in Fig 8, we notice the emergent logarithmic scale when the only singular frequency is $\omega=0$. This logarithmic scale is a characteristic feature of the renormalization group (RG) methods [65]. The interesting difference is that the traditional (equilibrium-inspired) RG methods perform the logarithmic discretization of the energy band, whereas in our case it is the time behaviour which scales logarithmically. However the core principle is the same: (i) there is a flow, (ii) as we proceed along the flow, only the low-energy behaviour remains to be relevant, see Fig. 10 In the equilibrium-inspired RG methods the flow is the decreasing characteristic energy cut-off. In our case the flow is the "aging" (moving to the past in time) of the emitted field, and the relevant low-energy behaviour is the progressively small vicinity of the singular frequencies in spectral function $J(\omega)$, as in Fig. 3 .

\section{The emergence of outgoing modes in real time}

In this section we implement the intuition of Fig 4 and Fig. 10: we construct the stream of outgoing modes. That is, for each infinitesimal motion $\tau_{p} \rightarrow \tau_{p+1}$, we identify the new mode $\phi_{\text {out }}(p)$ of the environment that has just become irreversibly decoupled (which belongs to the gray area in Fig 4 and to the shell $D_{p}$ in Fig. 10).

We construct the stream of outgoing modes by induction. Suppose we have chosen to keep no more than $m$ relevant modes. This is how we demarkate the boundary of the salmon-colored area in Fig. 3. This means that for $\tau_{p}$ with $p \leq m-1$ all the modes are relevant since the matrix $K(p)$ has no more than $m$ eigenvalues. This is the basis for our inductive construction.

Now suppose that at a time moment $\tau_{p}, p \geq m-1$, we know what are the relevant modes $\phi_{1}(p), \ldots, \phi_{m}(p)$, and know what are (zero or more) outgoing modes $\phi_{\text {out }}(p-1), \phi_{\text {out }}(p-2), \ldots, \phi_{\text {out }}(m)$, see Fig. 11, a). At the time moment $\tau_{p}$ the open system (the write/erase head in our tape model) points to the cell $\tau_{p}$. This cell represents one incoming mode $\phi_{\text {in }}(p)$ which is localized on this cell: $\left(r \mid \phi_{\text {in }}(p)\right)=\delta_{r p}$, with $\delta$ being the Kronecker delta.

The inductive step in our construction is to consider what happens after the propagation to the next time moment $\tau_{p} \rightarrow \tau_{p+1}$. The incoming mode $\phi_{\text {in }}(p)$ becomes one of the past cells (the write/erase head moves one position to the left). As a result, by the time moment $\tau_{p+1}$ we have the following $m+1$ modes: $\phi_{1}(p), \ldots, \phi_{m}(p)$ and $\phi_{\text {in }}(p)$. Our task to is to transform these modes into the new $m$ relevant modes $\phi_{1}(p+1), \ldots, \phi_{m}(p+1)$, and one new outgoing mode $\phi_{\text {out }}(p)$,

$$
\left[\begin{array}{c}
\phi_{1}(p+1) \\
\vdots \\
\phi_{m}(p+1) \\
\phi_{\text {out }}(p)
\end{array}\right]=W_{p}\left[\begin{array}{c}
\phi_{1}(p) \\
\vdots \\
\phi_{m}(p) \\
\phi_{\text {in }}(p)
\end{array}\right]
$$

via some unitary transform $W_{p}$. Here $W_{p}$ acts as ordinary matrix on the column entries e.g. $\phi_{k}(p+1)=$ $\sum_{l}\left(W_{p}\right)_{k l} \phi_{l}(p)+\left(W_{p}\right)_{k, m+1} \phi_{\text {in }}(p)$. In order to determine $W_{p}$, we choose the fastest decoupling basis. As the fastest decoupling basis we take the eigenvectors of $K(p+1)$ in the space spanned by $\phi_{1}(p), \ldots, \phi_{m}(p)$ and $\phi_{\text {in }}(p)$. The matrix elements of $K(p+1)$ in this subspace are:

$$
\widetilde{K}(p+1)=\left[\begin{array}{ll}
K_{\mathrm{rr}} & K_{\mathrm{ri}} \\
K_{\mathrm{ri}}^{\dagger} & K_{\mathrm{ii}}
\end{array}\right]
$$

where the blocks of the matrix are: the scalar $K_{\mathrm{ii}}=\left(\phi_{\text {in }}(p)|K(p+1)| \phi_{\text {in }}(p)\right)=$ $K(p+1)_{p p}=\sum_{p^{\prime}=1}^{\infty}\left|M\left(p^{\prime} d t\right)\right|^{2} ; \quad$ the vector $\left(K_{\mathrm{ri}}\right)_{q} \stackrel{p p}{=}\left(\phi_{\text {in }}(p)|K(p+1)| \phi_{q}(p)\right) ; \quad$ the matrix $\left(K_{\mathrm{rr}}\right)_{q q^{\prime}}=\left(\phi_{q}(p)|K(p+1)| \phi_{q^{\prime}}(p)\right)$. This matrix has dimension $(m+1) \times(m+1)$. Therefore, if we diagonalize it and sort the $m+1$ eigenvectors in the descending order of their eigenvalues,

$$
\widetilde{K}(p+1)=U\left[\begin{array}{ccc}
\lambda_{1} & & 0 \\
& \ddots & \\
0 & & \lambda_{m+1}
\end{array}\right] U^{\dagger}
$$

we obtain $W_{p}=U^{T}$ for eq. (21).

Iterating this inductive step, we construct the entire stream of outgoing modes $\phi_{\text {out }}(p)$, see Fig. 12 

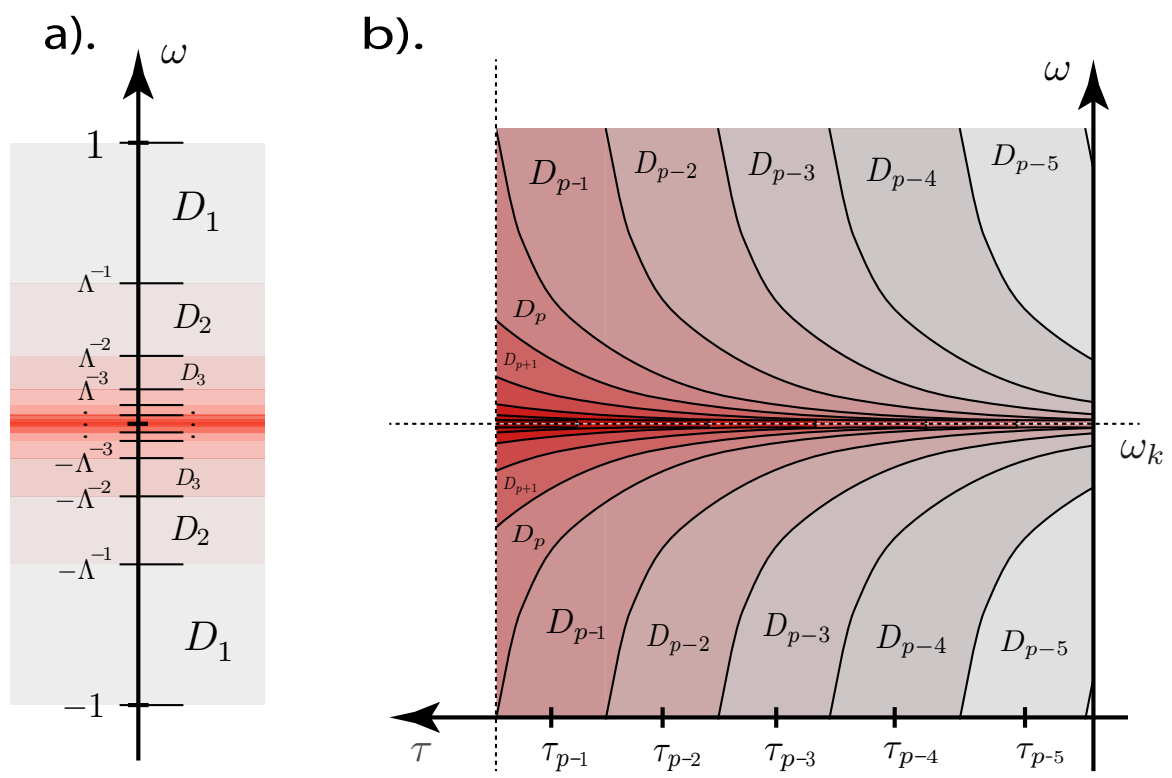

Figure 10. Comparision between the traditional "equilibruim-inspired" and the real-time renormalization groups (RG). a). In the traditional RG, we have initial energy interval $[-1,1]$ (in some arbirtary units). Then one makes assumption that only the low energy behaviour is relevant for the problem at hand. The logarithmic discretization of the energy interval is introduced with $\Lambda>$ 1. We obtain an infinite sequence of energy shells $D_{1}=\left[-1,-\Lambda^{-1}\right] \cup\left[\Lambda^{-1}, 1\right], \ldots, D_{k}=\left[-\Lambda^{-k+1},-\Lambda^{-k}\right] \cup\left[\Lambda^{-k}, \Lambda^{-k+1}\right], \ldots$ . The renormalization group flow is obtained by sequentially tracing out the degrees of freedom in the shells $D_{1}, D_{2}, \ldots D_{k}, \ldots \ldots$ b). In the real-time renormalization group, the relevant degrees of freedom are contained in the progressively small vicinity of each singular frequency $\omega_{k}$ where the spectral density $J(\omega)$ has sharp features (e.g. band edges). Thus we obtain the sequence of phase-space shells $D_{p-1}, D_{p}, D_{p+1}, \ldots$. The shell $D_{k}$ contains the spectral content which decouples from future evolution after the time moment $\tau_{k}$, see Fig. 4. Each shell $D_{k}$ carries a degree of freedom $\phi_{\text {out }}(k)$, which are constructed in sec. IV D. These $\phi_{\text {out }}(k)$ are traced out in sec. VI and collapsed to classical noise in sec. VIII.

\section{EQUATIONS OF MOTION IN TERMS OF INCOMING, OUTGOING, AND RELEVANT MODES}

In this section we give a precise meaning to the Fig. 1. In the previous section the treatment was on the level of superpositios of single-quantum states of the environment. Here we derive the complete quantum equations of motions, which fully take into account the many-body correlations between the open quantum system and the environment.

We remind you that our goal is to find a minimum number of degrees of freedom which contain everything what is significant for the motion at future time moments. We want to stay in the small relevant subspace (spanned by these degrees of freedom) all the time. However during each infinitesimal time interval the quantum amplitudes leak outside this space (due to the environment dispersion). In other words, at least one new degree of freedom gets entangled. In order to keep the minimal number of relevant modes, we need to trace out some degree of freedom. This degree of freedom should be independent and decoupled from everything. Otherwise, the real-time motion will become corrupted on large times. We conclude that we need to attach an independent degree of free- dom to each infinitesimal time moment. Otherwise there will be nothing to trace out, and the number of coupled modes will grow with time. Therefore, in this section we develop a formalism which treats the infinitesimal time cells as independent degrees of freedom. We then map the model of open quantum system (52) to the representation of independent time cells ("tape model") in an exact and rigorous way.

\section{A. Fock space for the tape cells}

Each cell $\tau_{p}$ of the time tape (the light turquoise circles in Figs. 2, 5, and 6) is considered as independent bosonic degree of freedom with its own creation operator $\widehat{\psi}_{p}^{\dagger},\left[\widehat{\psi}_{q}, \widehat{\psi}_{p}^{\dagger}\right]=\delta_{q p}$. The operator $\widehat{\psi}_{p}^{\dagger}$ creates a quantum in the mode $\phi_{\text {in }}(p)$. Recalling Fig. 2, this implies that we map the creation operator $\widehat{b}^{\dagger}\left(\tau_{p}\right)$ to $\widehat{\psi}_{p}^{\dagger}$,

$$
\widehat{b}^{\dagger}\left(\tau_{p}\right) \rightarrow \widehat{\psi}_{p}^{\dagger}
$$

As a result, the tape has its own vacuum $|0\rangle_{\mathrm{t}}$ and the tape Fock space $\mathcal{F}_{\mathrm{t}}$ is spanned by applying $\widehat{\psi}_{p}^{\dagger}$ 's to $|0\rangle_{\mathrm{t}}$. This Fock space is formally different from that $\mathcal{F}_{\mathrm{b}}$ defined by 

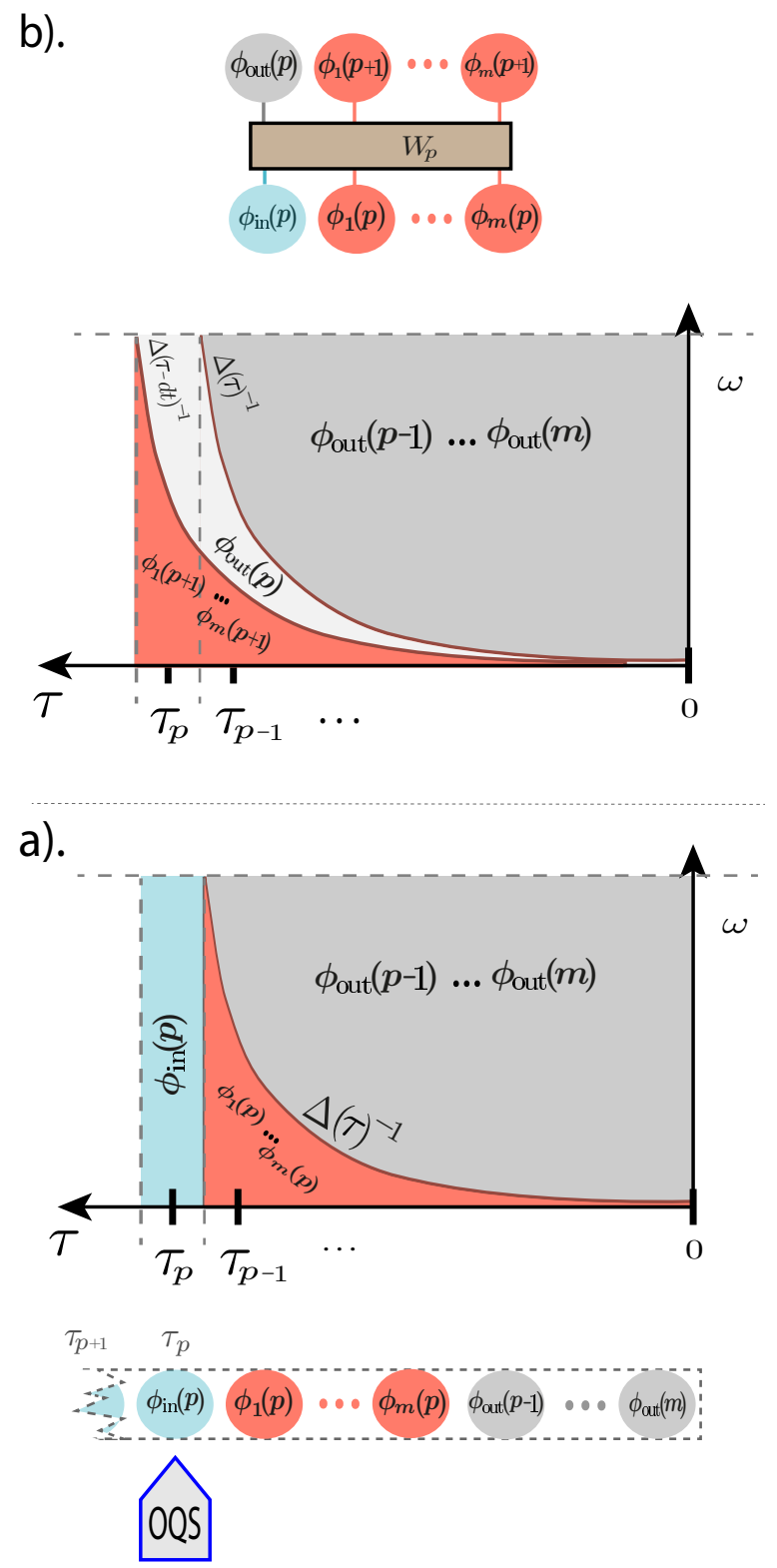

Figure 11. a). At a time moment $\tau_{p}$, the open system gets coupled to the new incoming mode $\phi_{\text {in }}(p)$. Since this mode is localized (in $\tau$ ), it has a broadband spectrum (the vertical light turquoise spectral area). The past cells are represented by the relevant modes $\phi_{1}(p), \ldots, \phi_{m}(p)$, which carry the coupled part of the emitted quantum field (salmon-colored spectral area, under the decreasing scale $\left.\Delta(\tau)^{-1}\right)$. There are also outgoing modes $\phi_{\text {out }}(p-1), \phi_{\text {out }}(p-2), \ldots, \phi_{\text {out }}(m)$, which represent the irreversibly decoupled part of the emitted quantum field (the gray spectral area). b). After the propagation to the next time moment $\tau_{p} \rightarrow \tau_{p+1}$, the incoming mode $\phi_{\text {in }}(p)$ becomes one of the past cells. Then the scale $\Delta(\tau)^{-1}$ shifts to the left, $\Delta(\tau)^{-1} \rightarrow \Delta(\tau-d t)^{-1}$, as described in Fig. 4. This leads to the emergence of a new decoupled spectral content (light gray area). This spectral content is represented by a new outgoing mode $\phi_{\text {out }}(p)$. The latter is found via a unitary transform $W_{p}$, eq. (21), to the frame of the new relevant modes $\phi_{1}(p+1), \ldots, \phi_{m}(p+1)$.

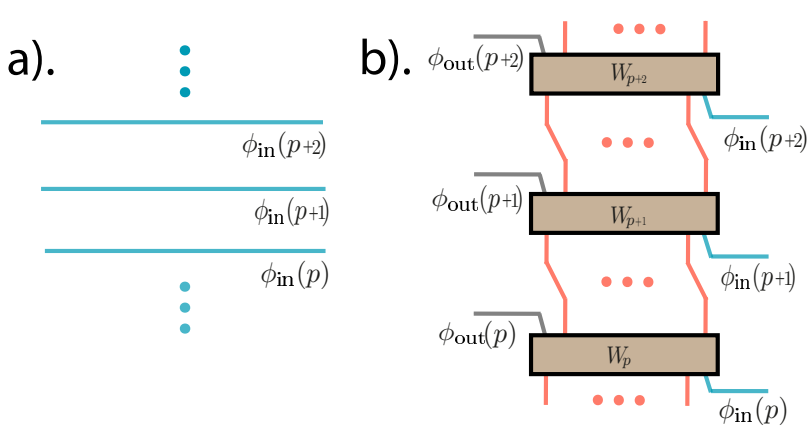

Figure 12. a). The tape model of real-time evolution assigns an incoming mode $\phi_{\text {in }}(p)$ to each time moment $\tau_{p}$. b). The iterative construction of the $m$ most relevant modes changes the structure of modes: now we have the two streams of incoming $\phi_{\text {in }}(p)$ (turquoise lines) and outgoing modes $\phi_{\text {out }}(p)$ (gray lines), and also the relevant modes $\phi_{1}(p) \ldots \phi_{m}(p)$ (salmoncolored lines), which represent the corresponding spectral areas of Figs. 4 and 11.

the original model in eq. (4). However the quantum mechanics permits many different formal representations of a physical system, provided that the observable properties are invariant. For the latter it is enough to conserve the commutation relation (8), which is ensured by the mapping

$$
\widehat{b}\left(\tau_{l}\right) \rightarrow \sum_{r=0}^{l} M_{l r} \widehat{\psi}_{r}
$$

were we again employ the matrix $M$ with elements $M_{l r}=M((l-r) d t)$. Now the tape model and the original model eq. (4) are in one-to-one correspondence. For example, the states are mapped between these models as:

$$
\begin{aligned}
& \widehat{\psi}_{0}^{\dagger n_{0}} \ldots \widehat{\psi}_{p-1}^{\dagger n_{p-1}}\left|\phi\left(n_{0}, \ldots n_{p-1}\right)\right\rangle_{\mathrm{s}} \otimes|0\rangle_{\mathrm{t}} \\
& \longleftrightarrow \widehat{b}^{\dagger n_{0}}\left(\tau_{0}\right) \ldots \widehat{b}^{\dagger n_{p-1}}\left(\tau_{p-1}\right)\left|\phi\left(n_{0}, \ldots n_{p-1}\right)\right\rangle_{\mathrm{s}} \otimes|0\rangle_{\mathrm{b}}
\end{aligned}
$$

Here $\left|\phi\left(n_{0}, \ldots n_{p-1}\right)\right\rangle_{\mathrm{s}}$ is a wavefunction of the open system which is not affected by the mapping. The vacuum initial condition for the tape model becomes $|\Phi(0)\rangle_{\mathrm{st}}=$ $\left|\phi_{0}\right\rangle_{\mathrm{s}} \otimes|0\rangle_{\mathrm{t}}$.

\section{B. The Hamiltonian for the tape model}

The Hamiltonian for the tape model is obtained by applying the mapping rules (24)- (25) to the original Hamitonian (4):

$$
\widehat{H}_{\mathrm{sb}}\left(\tau_{p}\right) \rightarrow \widehat{H}_{\mathrm{st}}\left(\tau_{p}\right)=\widehat{H}_{\mathrm{s}}+\widehat{s} \widehat{\psi}_{p}^{\dagger}+\widehat{s}^{\dagger} \sum_{r=0}^{p} M_{p r} \widehat{\psi}_{r} .
$$

Observe that in the tape model the real-time evolution happens in discrete time steps, via jumps from one cell $\tau_{p}$ 
to the neighboring one $\tau_{p+1}$. Of course, we want this discrete evolution to approximate the continuous one from the original model (4). It is desirable that the global propagation error is stable and vanishing as $d t \rightarrow 0$. The latter can be achieved by employing the implicit midpoit rule as a propagator,

$$
\begin{aligned}
\left|\Phi\left(t_{p+1}\right)\right\rangle_{\mathrm{st}}=\left|\Phi\left(t_{p}\right)\right\rangle_{\mathrm{st}} & \\
-i d t \widehat{H}_{\mathrm{st}}\left(\tau_{p}\right) \frac{1}{2}\left\{\left|\Phi\left(t_{p+1}\right)\right\rangle_{\mathrm{st}}+\right. & \left.\left|\Phi\left(t_{p}\right)\right\rangle_{\mathrm{st}}\right\} \\
& +O\left(d t^{3}\right),
\end{aligned}
$$

where now we specify that the tape cells are located at the midpoint times: $\tau_{p}=\left(p+\frac{1}{2}\right) d t$. This equation is solved for $\left|\Phi\left(t_{p+1}\right)\right\rangle_{\text {st }}$ by iterations with the initial guess $\left|\Phi\left(t_{p+1}\right)\right\rangle_{\mathrm{st}}=\left|\Phi\left(t_{p}\right)\right\rangle_{\mathrm{st}}$. Here the Hamiltonian $\widehat{H}_{\mathrm{sb}}\left(\tau_{p}\right)$ corresponds to the head (open system) being coupled to the cell $\tau_{p}$. Then the midpoint rule enforces us to think that the Hamiltonian $\widehat{H}_{\mathrm{sb}}\left(\tau_{p}\right)$ generates the evolution from the wavefunction $\left|\Phi\left(t_{p}\right)\right\rangle_{\mathrm{st}}$ at the time moment $t_{p}=p d t$ to the wavefunction $\left|\Phi\left(t_{p+1}\right)\right\rangle_{\text {st }}$ at the next time moment $t_{p+1}=(p+1) d t$. This ensures the global error of $O\left(d t^{2}\right)$. The time moment $t_{p}$ corresponds to the notation of Fig. 1.

The wavefunction $\left|\Phi\left(t_{p}\right)\right\rangle_{\text {st }}$ depends on the modes $\widehat{\psi}_{0}^{\dagger}, \ldots, \widehat{\psi}_{p-1}^{\dagger}$. During each propagation step of eq. (28) it entangles one additional mode $\widehat{\psi}_{p}^{\dagger}$ (which corresponds to $\phi_{\text {in }}(p)$ ), see Fig 13. This is the usual growth of complexity which we overcome in this work.

\section{Partial trace}

To completely define the tape model, we need to discuss how to compute the trace over the cell degrees of freedom. Suppose we compute the reduced density matrix for open system via the partial trace over the environment:

$$
\widehat{\rho}_{\mathrm{s}}\left(t_{p}\right)=\operatorname{Tr}_{\mathrm{b}}\left\{\left|\Phi\left(t_{p}\right)\right\rangle_{\mathrm{sb} \mathrm{sb}}\left\langle\Phi\left(t_{p}\right)\right|\right\},
$$

where $\left|\Phi\left(t_{p}\right)\right\rangle_{\mathrm{sb}}$ is the wavefunction of the original model (4). The effect of $\operatorname{Tr}_{\mathrm{b}}$ is that each $\widehat{a}^{\dagger}(\omega)$ from the ket $\left|\Phi\left(t_{p}\right)\right\rangle_{\mathrm{sb}}$ becomes paired with $\widehat{a}(\omega)$ 's from the bra ${ }_{\mathrm{sb}}\left\langle\Phi\left(t_{p}\right)\right|$ at the same frequency. This follows from the commutation relations $\left[\widehat{a}(\omega), \widehat{a}^{\dagger}\left(\omega^{\prime}\right)\right]=\delta\left(\omega-\omega^{\prime}\right)$. We can rewrite the partial trace (29) in such a form that all the pairings become explicit:

$$
\begin{aligned}
\widehat{\rho}_{\mathrm{S}}\left(t_{p}\right)={ }_{\mathrm{b}}\langle 0| & \left\{e^{\int_{0}^{+\infty} d \omega \widehat{a}^{\dagger}(\omega) \widehat{a}(\omega)}\right. \\
& \left.\times:\left|\Phi\left(t_{p}\right)\right\rangle_{\mathrm{sb} \mathrm{sb}}\left\langle\Phi\left(t_{p}\right)\right|:\right\}_{A}|0\rangle_{\mathrm{b}} .
\end{aligned}
$$

Here the notation $\{::\}_{A}$ means that the creation/annihilation operators coming from the Taylor expansion of the pairing function $\exp \int_{0}^{+\infty} d \omega \widehat{a}^{\dagger}(\omega) \widehat{a}(\omega)$ are antinormally ordered around the term

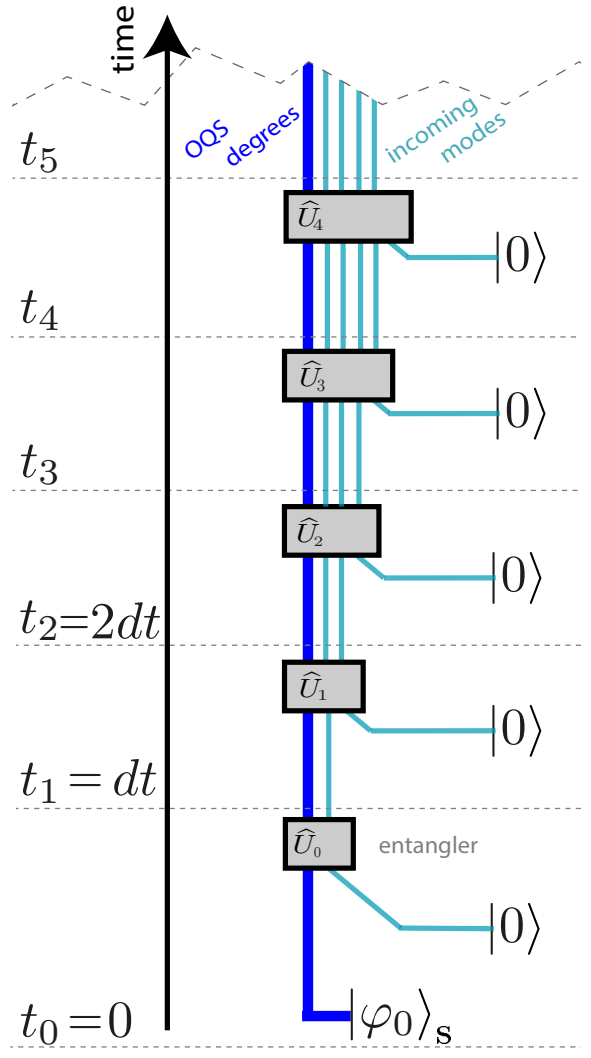

Figure 13. In the tape model of open system the evolution happes between the discrete time moments $t_{0}=0$, $t_{1}=d t, \ldots t_{p}=p d t, \ldots$. After propagation from $t_{p}$ to $t_{p+1}$, one new incoming mode $\phi_{\text {in }}(p)$ (turquoise line) corresponding to the cell $\tau_{p}$ becomes entangled to the open system (blue line). The evolutions $\widehat{U}_{p}$ happen under the midpoint rule (28).

$\left|\Phi\left(t_{p}\right)\right\rangle_{\mathrm{sb} \mathrm{sb}}\left\langle\Phi\left(t_{p}\right)\right|$. The ordering of the latter term is not affected (which is indicated by placing it between $:$ : ). Observe that when we order the Taylor expansion of the pairing function, we regard its constituent creation/annihilation operators as commuting. The expressions (29) and (30) are equal because they describe the same set of pairings between the $\widehat{a}^{\dagger}(\omega)$ 's from the ket $\left|\Phi\left(t_{p}\right)\right\rangle_{\mathrm{sb}}$, and the $\widehat{a}(\omega)$ 's from the bra ${ }_{\mathrm{sb}}\left\langle\Phi\left(t_{p}\right)\right|$. The operation ${ }_{b}\langle 0|\cdot| 0\rangle_{\mathrm{b}}$ denotes the projection of density matrix to the subspace of zero quanta in the environment. This operation does not affect the open system.

When defining the trace for the wavefunction $\left|\Phi\left(t_{p}\right)\right\rangle_{\text {st }}$ of the tape model, we should take into account that each pairing between $\widehat{\psi}_{r}$ and $\widehat{\psi}_{s}^{\dagger}$ is possible due to the commutator $\left[\widehat{b}\left(\tau_{r}\right), \widehat{b}^{\dagger}\left(\tau_{s}\right)\right]=M_{r s}$ :

$$
\begin{aligned}
\widehat{\rho}_{\mathrm{S}}\left(t_{p}\right)={ }_{\mathrm{t}}\langle 0|\left\{e^{\sum_{r s=0}^{p-1} \widehat{\psi}_{r}^{\dagger} M_{r s} \widehat{\psi}_{s}}\right. & \\
& \left.\times:\left|\Phi\left(t_{p}\right)\right\rangle_{\mathrm{st} \mathrm{st}}\left\langle\Phi\left(t_{p}\right)\right|:\right\}_{A}|0\rangle_{\mathrm{t}} .
\end{aligned}
$$

This is how the partial trace is performed in the tape 
model. The conceptual meaning of the pairing function is that the time-cell degrees of freedom yield non-local contribution to the observables in quantum mechanics, see Fig. 14

\section{Propagation of wavefunction in terms of incoming, outgoing, and relevant modes}

The initial condition for the propagation is $|\Phi(0)\rangle_{\text {st }}=$ $\left|\phi_{0}\right\rangle_{\mathrm{s}} \otimes|0\rangle_{\mathrm{t}}$.

There are two regimes of propagation: $t_{p}<t_{m}$ and $t_{p} \geq t_{m}$.

As was discussed in sec. IVD, while $t_{p}<t_{m}$, all the modes $\widehat{\psi}_{0}^{\dagger} \ldots \widehat{\psi}_{p-1}^{\dagger}$ are relevant: $\phi_{\text {in }}(1) \equiv$ $\phi_{1}(p-1), \ldots, \phi_{\text {in }}(p-1) \equiv \phi_{p-1}(p-1)$. Therefore, the propagation from $\left|\Phi\left(t_{p}\right)\right\rangle_{\text {st }}$ to $\left|\Phi\left(t_{p+1}\right)\right\rangle_{\text {st }}$ is done via the application of the midpoint rule (28). As a result, before the time moment $t_{m}$ the entanglement develops according to the Fig. 13.

The situation changes for $t_{p} \geq t_{m}$. The wavefunction $\left|\Phi\left(t_{p}\right)\right\rangle_{\text {st }}$ depends on the relevant modes $\phi_{1}(p-1) \ldots \phi_{m}(p-1)$ via their creation operators $\widehat{\phi}_{1}^{\dagger}(p-1) \ldots \widehat{\phi}_{m}^{\dagger}(p-1)$. Also $\left|\Phi\left(t_{p}\right)\right\rangle_{\text {st }}$ depends on zero or more outgoing modes $\phi_{\text {out }}(p-1) \ldots \phi_{\text {out }}(m)$ via their creation operators $\widehat{\phi}_{\text {out }}^{\dagger}(p-1) \ldots \widehat{\phi}_{\text {out }}^{\dagger}(m)$, see Fig. 15 , a). Here each line represents the occupation number of the corresponding mode. The salmon-colored lines $\phi_{k} \equiv \phi_{k}(p-1), k=1 \ldots m$, correspond to the relevant modes which are populated via the creation operators $\widehat{\phi}_{k}^{\dagger}(p-1)$. The blue line denotes the quantum numbers of the open system. The gray lines are the outgoing modes.

The propagation begins with entangling step: when the discrete-time Hamiltonian $\widehat{H}_{\mathrm{st}}\left(\tau_{p}\right)(27)$ is applied, the stage b). in Fig. 15. We express the Hamiltonian in terms of the incoming mode $\widehat{\psi}_{p}^{\dagger}$, the relevant modes $\widehat{\phi}_{k}^{\dagger}(p)$, and the irrelevant modes $\widehat{\phi}_{\text {out }}^{\dagger}(p)$ :

$$
\begin{aligned}
& \widehat{H}_{\mathrm{st}}\left(\tau_{p}\right)=\widehat{H}_{\mathrm{s}}+\widehat{s} \widehat{\psi}_{p}^{\dagger}+\widehat{s}^{\dagger} M(0) \widehat{\psi}_{p} \\
&+\widehat{s}^{\dagger} \sum_{i=1}^{\min (p, m)} M_{i}(p) \widehat{\phi}_{i}(p) \\
& \\
& \quad+\widehat{s}^{\dagger} \sum_{i=m+1}^{p-1} M_{\text {out } i}(p) \widehat{\phi}_{\text {out }}(i),
\end{aligned}
$$

where the coupling to the relevant modes is

$$
M_{i}(p)=\sum_{r=0}^{p-1} M_{p r} \phi_{i}(p)_{r},
$$

and the coupling to the irrelevant modes is $M_{\text {out } i}(p)=$ $\sum_{r=0}^{p-1} M_{p r} \phi_{\text {out }}\left(t_{i}\right)_{r}$. Up to now the treatement was exact (in the limit $d t \rightarrow 0$ ). Here we make an approximation for the first time: the coupling between the incoming

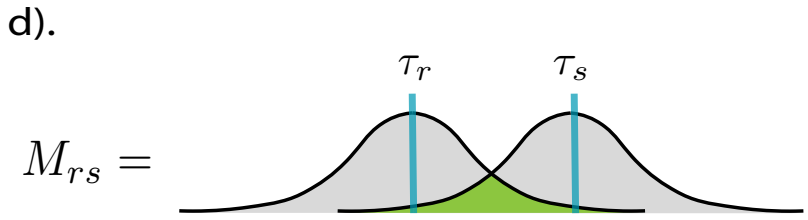

c).

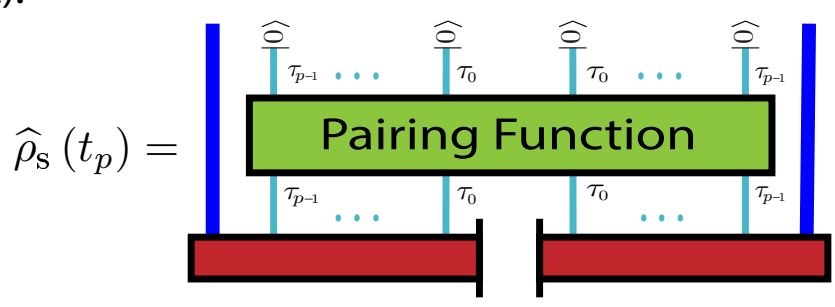

b).

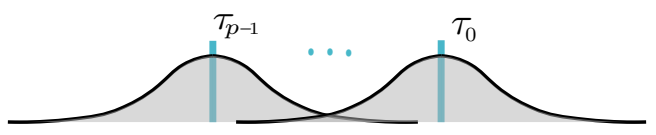

a).

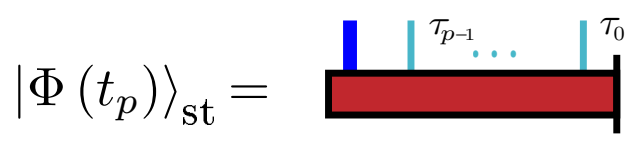

Figure 14. a). It is very desirable to consider the wavefunction as depending on quantum numbers of each infinitesimal time moment (turquoise lines). This appoach is natural for analyzing how the entanglement gradually develops in real time. Especially it is interesting to track how is it happening continuously: what gets entangled and disentangled each infinitesimal time moment. We succeed in deriving equations of motion which treat the infinitesimal time cells as independent degrees of freedom: see eqs. (27) and (28). b). Nevertheless, there are no time-local states in quantum mechanincs. Due to the commutation relation (8), each time cell $\tau_{k}$ contributes to the observables at another time moment $t$ like a wavepacket $M_{\tau_{k} t}^{1 / 2}$ with long-range tails. Here $M_{\tau_{k} t}^{1 / 2}=(2 \pi)^{-\frac{1}{2}} \int_{0}^{+\infty} d \omega e^{-i \omega\left(\tau_{k}-t\right)} \sqrt{J(\omega)}$. c). We reconcile the dynamical locality and the observable non-locality by introducing the pairing function. This effectively smears the time cells in time during the partial trace operation. The resulting density matrix $\widehat{\rho}_{\mathrm{s}}\left(t_{p}\right)$ depends only on quantum numbers of the open system (blue lines). d). The elementary pairing $M_{r s}$ corresponds to the overlap (green area) between the wavepackets of the two quanta at $\tau_{r}$ and $\tau_{s}$ : $M_{r s}=\int_{-\infty}^{+\infty} M_{\tau_{r} t}^{1 / 2} M_{\tau_{s} t}^{1 / 2 *} d t$. 


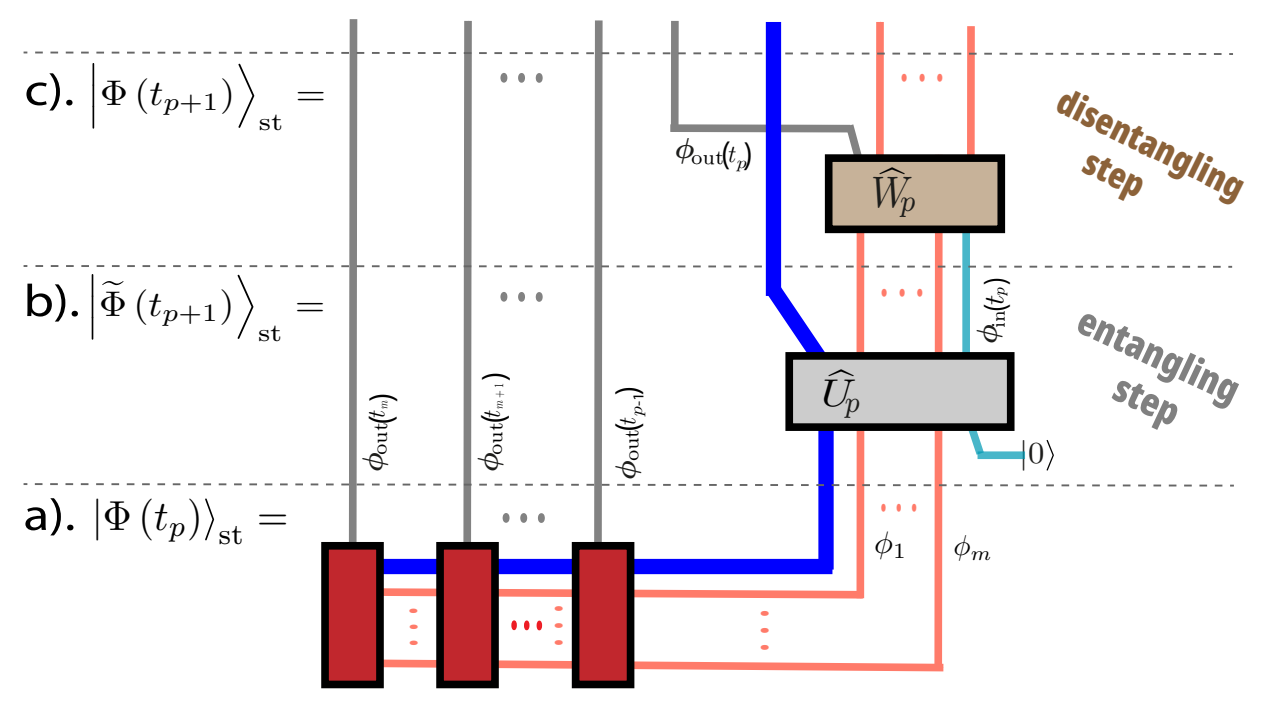

Figure 15. The circuit model of how the wavefunction is propagated during one time step in three stages: a). At a time moment $t_{p}$ the wavefunction $\left|\Phi\left(t_{p}\right)\right\rangle_{\text {st }}$ depends on the occupations of outgoing modes $\phi_{\text {out }}(k) \equiv \phi_{\text {out }}\left(t_{k}\right), k=m \ldots p-1$ (gray lines) and of relevant modes $\phi_{k} \equiv \phi_{k}(p), k=1 \ldots m$ (salmon lines). Also it depends on the quantum numbers of the open system (blue line). Observe the matrix-product-like structure of the wavefunction. This follows from the facts that (i) only $m$ modes are significantly coupled to the future and (ii) each time step one outgoing mode is produced. b). The discrete-time Hamiltonian (32) is applied via the midpoint rule (28), which approximates the evolution $\widehat{U}_{p}$. As a result, one incoming mode (turquoise line) gets entangled to the state. c). The frame is changed actively via $\widehat{W}_{p}$ to produce one decoupled mode (gray line). Observe that $\widehat{U}_{p}$ and $\widehat{W}_{p}$ constitute the newly formed additional block of the matrix product structure of $\left|\Phi\left(t_{p+1}\right)\right\rangle_{\mathrm{st}}$, see Fig. 16.

mode and the irrelevant modes (the last line of eq. (32)) is discarded, so that we have:

$$
\begin{aligned}
\widehat{H}_{\mathrm{st}}\left(\tau_{p}\right)=\widehat{H}_{\mathrm{s}}+\widehat{s} \widehat{\psi}_{p}^{\dagger} & +M(0) \widehat{\psi}_{p} \\
& +\widehat{s}^{\dagger} \sum_{i=1}^{\min (p, m)} M_{i}(p) \widehat{\phi}_{i}(p) .
\end{aligned}
$$

Below we will use this form of the Hamiltonian.

After the propagation $\widehat{U}_{p}$ via the rule $(28)$, the resulting wavefunction $\left|\widetilde{\Phi}\left(t_{p+1}\right)\right\rangle_{\text {st }}$ still depends on the "old" relevant modes $\widehat{\phi}_{k}^{\dagger}(p)$. The wavefunction $\left|\widetilde{\Phi}\left(t_{p+1}\right)\right\rangle_{\text {st }}$ also entangles one additional incoming mode $\widehat{\psi}_{p}^{\dagger}$ (the turquoise line going up from the stage b). in Fig. 15): hence the name "entangling step".

The propagation is finished by the disentangling step: we change to the basis of new relevant modes $\widehat{\phi}_{k}^{\dagger}(p+1)$, the stage c). in Fig. 15:

$$
\left|\Phi\left(t_{p+1}\right)\right\rangle_{\mathrm{st}}=\widehat{W}_{p}\left|\widetilde{\Phi}\left(t_{p+1}\right)\right\rangle_{\mathrm{st}},
$$

where the disentangler $\widehat{W}_{p}$ is given by

$$
\widehat{W}_{p}=\exp \left(i\left[\widehat{\phi}_{1}^{\dagger} \ldots \widehat{\phi}_{m}^{\dagger} \widehat{\psi}_{p}^{\dagger}\right] h(p)\left[\widehat{\phi}_{1} \ldots \widehat{\phi}_{m} \widehat{\psi}_{p}\right]^{T}\right),
$$

and the Hermitean $(m+1) \times(m+1)$ matrix $h(p)=$ $i \ln W_{p}$, with the unitary matrix $W_{p}$ defined in eq. (21).
Observe that in Eq. (35) we apply the active form of the frame-change transformation (36). As a result, the relevant modes loose their time dependence, $\widehat{\phi}_{k}^{\dagger}(p) \equiv$ $\widehat{\phi}_{k}^{\dagger}, k=1 \ldots m$. The resulting wavefunction $\left|\Phi\left(t_{p+1}\right)\right\rangle_{\mathrm{st}}$ has the same structure as $\left|\Phi\left(t_{p}\right)\right\rangle_{\mathrm{st}}$. Namely, it has the same matrix-product-state structure as in Fig. 15, a). Each block in this structure is formed by a pair of entangler-disentangler transforms, Fig. 16.

Observe that as the result of $\widehat{W}_{p}$, one additional outgoing mode $\phi_{\text {out }}(p)$ is produced in the wavefunction $\left|\Phi\left(t_{p+1}\right)\right\rangle_{\text {st }}$. The coupling of $\phi_{\text {out }}(p)$ to the future is negligible by construction, so that $\phi_{\text {out }}(p)$ will not become entangled to the future incoming modes: hence the name "disentangling step".

The iterative application of these propagation steps leads to the circuit model presented in Fig. 17.

\section{E. Decoupling of the pairing function}

Let us recall that when computing the observables, we need to apply the pairing function eq (31). It introduces an additional non-local coupling between the future and the past cell modes. One might wonder if this would spoil the entanglement structure described in Figs. $15-17$. The answer is no. The coupling in the pairing function has the same form of the convolutional annihilation process that was thoroughly analyzed in section IV. Therefore, in the frame of the incom- 
b).

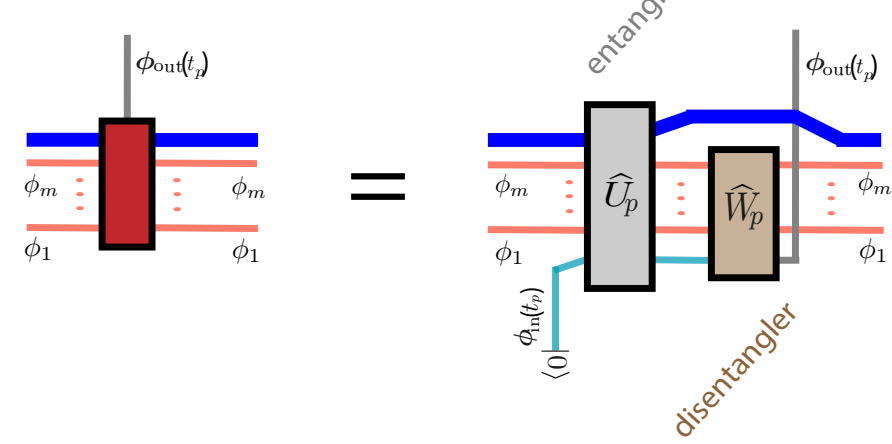

a).

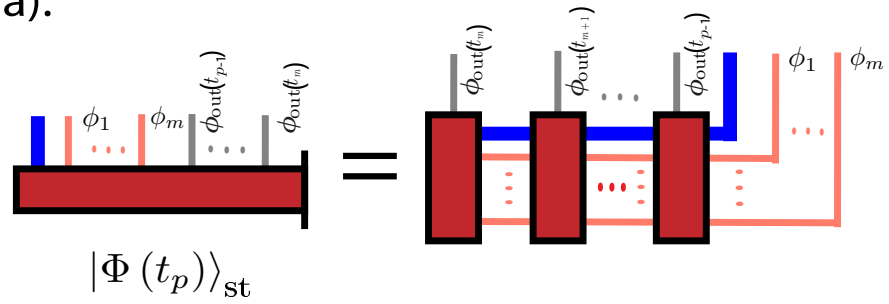

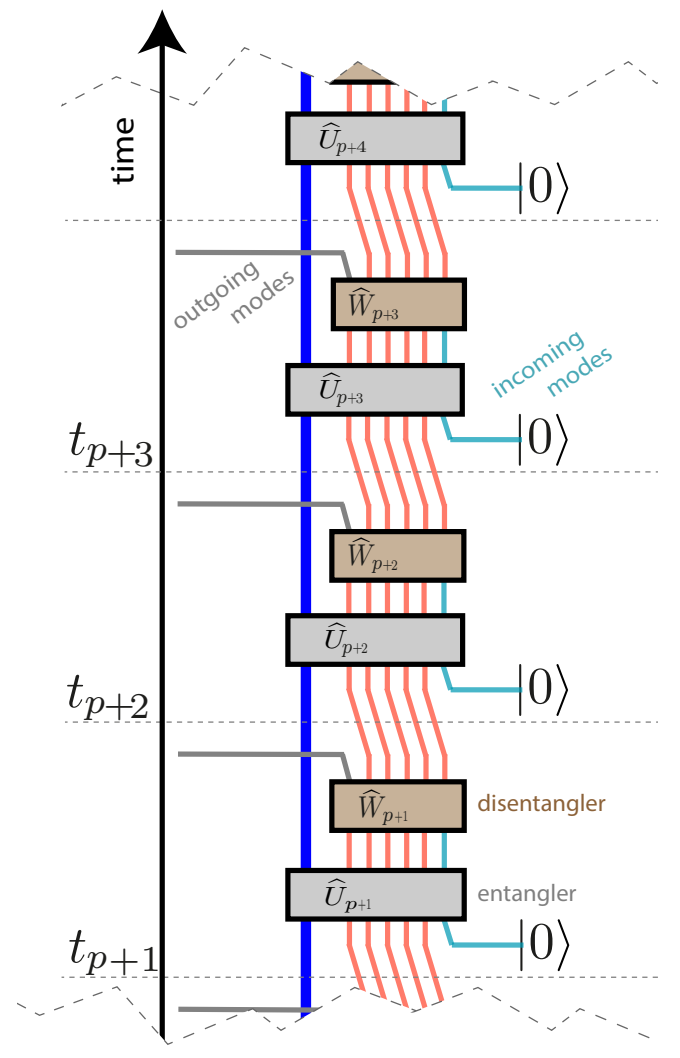

Figure 17. The circuit model of real-time evolution of open quantum system. Each time step $t_{p}$, one new incoming mode $\phi_{\text {in }}(p) \equiv \phi_{\text {in }}\left(t_{p}\right)$ get entangled to the open system via the Hamiltonian evolution $\widehat{U}_{p}$. Afterwards one new irreversibly decouled mode $\phi_{\text {out }}(p) \equiv \phi_{\text {out }}\left(t_{p}\right)$ gets disentangled via the active change of frame $\widehat{W}_{p}$. Observe that the product $\widehat{W}_{p} \widehat{U}_{p}$ is equal to $\widehat{U}_{p}^{\prime}$ from Fig. 1 .

operators are related in the same way:

$$
\widehat{\phi}^{\dagger} \equiv\left[\begin{array}{c}
\widehat{\phi}_{1}^{\dagger} \\
\vdots \\
\widehat{\phi}_{m}^{\dagger} \\
\widehat{\phi}_{\text {out }}^{\dagger}(m) \\
\vdots \\
\widehat{\phi}_{\text {out }}^{\dagger}(p-1)
\end{array}\right]=U\left[\begin{array}{c}
\widehat{\psi}_{0}^{\dagger} \\
\vdots \\
\widehat{\psi}_{p-1}^{\dagger}
\end{array}\right]
$$

where $\widehat{\phi}^{\dagger}$ is a shorthand for the column vector of creation operators. Its components $\widehat{\phi}_{1}^{\dagger} \ldots \widehat{\phi}_{m}^{\dagger}$ refer to $\widehat{\phi}_{1}^{\dagger} \ldots \widehat{\phi}_{m}^{\dagger}$ correspondignly, and $\widehat{\phi}_{m+1}^{\dagger} \ldots \widehat{\phi}_{m+p}^{\dagger}$ refer to $\widehat{\phi}_{\text {out }}^{\dagger}(m) \ldots \widehat{\phi}_{\text {out }}^{\dagger}(p-1)$ correspondingly. The inverse transforms are given by $U^{\dagger}$. We have for the pairing

where the unitary matrix $U$ is the cumulative effect of
the disentanglers: $U=W_{p} W_{p-1} \ldots W_{m}$. The creation 
a).

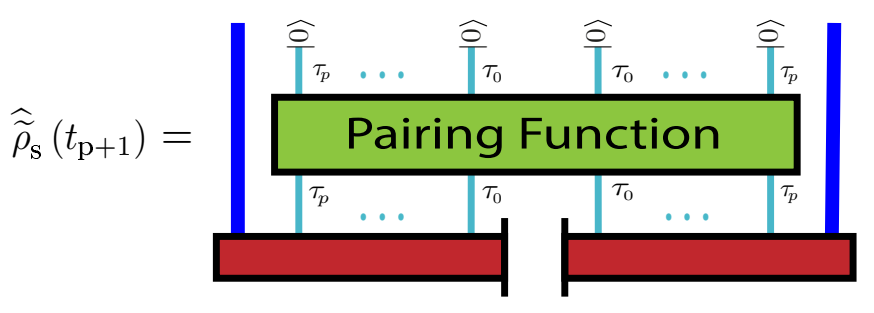

b).

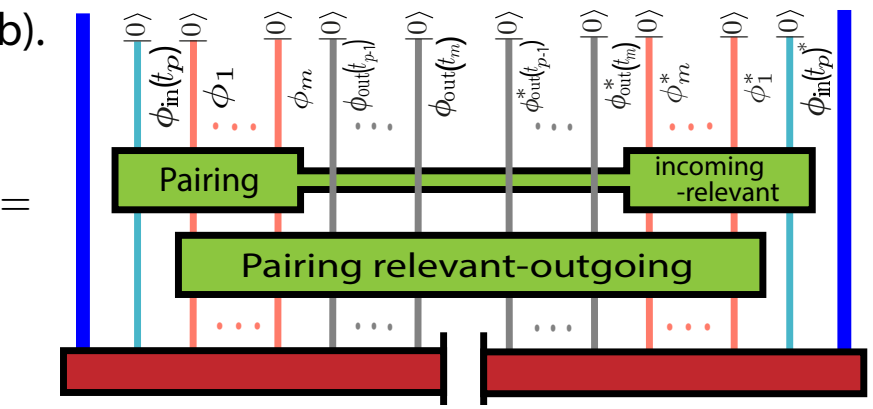

Figure 18. a). When evaluating observables or taking the partial trace, the pairing function introduces additional non-local coupling between the time cells. This coupling has the same convolutional form as the annihilation processes considered in sec. IV. b). Therefore, in the frame of incoming/relevant/outgoing modes, the couplings (i.e. pairings) between the past outgoing modes and the future incoming modes are still negligible. This is the foundation of our approach. The pairing function decouples into the two separate parts: pairings involving relevant-outoing modes and the pairings involving incoming-relevant modes.

function:

$$
\begin{aligned}
\sum_{r s=0}^{p-1} \widehat{\psi}_{r}^{\dagger} M_{r s} \widehat{\psi}_{s}=\sum_{r s=0}^{p-1} U_{r u}^{\dagger} \widehat{\boldsymbol{\phi}}_{u}^{\dagger} M_{r s} U_{s v}^{T} \widehat{\boldsymbol{\phi}}_{v} \\
=\sum_{u v}^{p-1} \widehat{\boldsymbol{\phi}}_{u}^{\dagger} \widetilde{M}_{u v}(p) \widehat{\boldsymbol{\phi}}_{v}
\end{aligned}
$$

where the matrix $\widetilde{M}$ has the block structure which reflects the pairings between the relevant and ougoing modes:

$$
\widetilde{M}(p)=\left[\begin{array}{ll}
M_{\mathrm{rr}} & M_{\mathrm{ro}} \\
M_{\mathrm{ro}}^{\dagger} & M_{\mathrm{oo}}
\end{array}\right] .
$$

Here $M_{\mathrm{rr}}$ generate the pairings between the relevant modes: $\left(M_{\mathrm{rr}}\right)_{r s}=\left(\phi_{r}(p)|M| \phi_{s}(p)\right) ; \quad\left(M_{\mathrm{oo}}\right)_{r s}=$ $\left(\phi_{\text {out }}(r)|M| \phi_{\text {out }}(s)\right)$ generates the pairings between the outgoing modes; $\left(M_{\text {ro }}\right)_{r s}=\left(\phi_{r}(p)|M| \phi_{\text {out }}(s)\right)$ defining the pairings between the relevant-outgoing modes.

\section{Negligible pairings between the past outgoing and the future incoming modes}

Now suppose we have a new incoming mode $\phi_{\text {in }}(p)$ with the creation operator $\widehat{\psi}_{p}^{\dagger}$. It will yield additional terms in the pairing function:

$$
\begin{gathered}
\widehat{\psi}_{p}^{\dagger} M(0) \widehat{\psi}_{p}+\sum_{s=0}^{p-1}\left\{\widehat{\psi}_{p}^{\dagger} M_{p s} \widehat{\psi}_{s}+\widehat{\psi}_{s}^{\dagger} M_{s p} \widehat{\psi}_{p}\right\} \\
=\widehat{\psi}_{p}^{\dagger} M(0) \widehat{\psi}_{p} \\
+\widehat{\psi}_{p}^{\dagger}\left\{\sum_{i} M_{i}(p) \widehat{\phi}_{i}+\sum_{i} M_{\text {out } i}(p) \widehat{\phi}_{\text {out }}(i)\right\}+\text { c.c.. }
\end{gathered}
$$

The last term in the second line, $M_{\text {out } i}(p) \widehat{\phi}_{\text {out }}(i)$, can be discarded by construction of the streams of outgoing modes. Therefore, when taking the partial trace over the time cells, we can assume that there are no pairings between the past outgoing and the future incoming modes, and the described above structure of entanglement, Fig. 17 , is preserved.

\section{TRACING OUT THE OUTGOING MODES: RENOMALIZATION GROUP FOR DENSITY MATRICES}

In this section we implement the idea that the "idle" bricks in Fig. 1 can be removed as soon as they emerge. In the conventional RG approach, we trace out the irrelevant degrees of freedom as soon as they appear. Therefore, in this section we introduce the joint density matrix $\widehat{\rho}_{\text {rel }}\left(t_{p}\right)$ of the open system and of the relevant degrees of freedom of the environment as a partial trace over the irrelevant degrees. After that an iterative propagation procedure is derived for $\widehat{\rho}_{\text {rel }}\left(t_{p}\right)$.

\section{A. The convention of growing Fock space}

We consider the case of the vacuum initial condition for the environment. In this case the incoming modes are always in vacuum. Then the notation will be more concise if we assume that the wavefunction $\left|\Phi\left(t_{p}\right)\right\rangle_{\mathrm{st}}$ is defined on a growing Hilbert space $\mathcal{H}_{\mathrm{s}} \otimes \mathcal{F}_{\mathrm{t}}(p)$ of the open system, the present and the past tape cells. Namely, at $t_{0}=0$ there are no cells at all, so that $\mathcal{F}_{\mathrm{t}}(0)=\oslash$, and the initial condition is just $|\Phi(0)\rangle_{\mathrm{st}}=\left|\phi_{0}\right\rangle_{\mathrm{s}}$. After each propagation $t_{p} \rightarrow t_{p+1}$, one additional incoming mode $\psi_{p}^{\dagger}$ gets coupled, and the Fock space is enlarged: $\mathcal{F}_{\mathrm{t}}(p+1)=\mathcal{F}_{\mathrm{t}}(p) \otimes\left\{\right.$ space spanned by $\left.\psi_{p}^{\dagger}\right\}$. Below we follow this convention. 


\section{B. Density matrix in the time domain}

We recall the formula eq. (31) for the partial trace over the time cells. We can rewrite it as

$$
\widehat{\rho}_{\mathrm{s}}\left(t_{p}\right)={ }_{\mathrm{t}}\left\langle 0\left|\widehat{\rho}_{\mathrm{st}}\left(t_{p}\right)\right| 0\right\rangle_{\mathrm{t}},
$$

where we introduce the joint density matrix for the open system and the time cells,

$$
\begin{aligned}
& \widehat{\rho}_{\mathrm{st}}\left(t_{p}\right) \\
& \quad=\left\{e^{\sum_{r s=0}^{p-1} \widehat{\psi}_{r}^{\dagger} M_{r s} \widehat{\psi}_{s}}:\left|\Phi\left(t_{p}\right)\right\rangle_{\mathrm{st} \mathrm{st}}\left\langle\Phi\left(t_{p}\right)\right|:\right\}_{A},
\end{aligned}
$$

see also Fig 19. This density matrix is Hermitean and positive semidefinite. The latter follows from the fact that $\left|\Phi\left(t_{p}\right)\right\rangle_{\text {st st }}\left\langle\Phi\left(t_{p}\right)\right|$ is positive semidefinite, and the action of the pairing function is a completely positive map since it has the "sandwitch" form required by the Stinespring factorization theorem. Indeed, each elementary pairing has the form

$$
\sum_{r s} M_{r s} \widehat{\psi}_{s}: \ldots: \widehat{\psi}_{r}^{\dagger}=\int_{-\infty}^{+\infty} d \tau \widehat{K}(\tau): \ldots: \widehat{K}^{\dagger}(\tau),
$$

where $\widehat{K}(\tau)=\sum_{s} M_{\tau \tau_{S}}^{1 / 2} \widehat{\psi}_{s}$, and $M_{\tau \tau^{\prime}}^{1 / 2}=$ $(2 \pi)^{-\frac{1}{2}} \int_{0}^{+\infty} d \omega e^{-i \omega\left(\tau-\tau^{\prime}\right)} \sqrt{J(\omega)}$ is the wavepacket of a single emission event from Fig. 14 .

However the density matrix $\widehat{\rho}_{\text {st }}\left(t_{p}\right)$ need not be normalized in the conventional sense, $\operatorname{Tr} \widehat{\rho}_{\text {st }}\left(t_{p}\right) \neq 1$. Instead, its vaccum part is always normalized:

$$
\operatorname{Tr}\left\{{ }_{\mathrm{t}}\left\langle 0\left|\widehat{\rho}_{\mathrm{st}}\left(t_{p}\right)\right| 0\right\rangle_{\mathrm{t}}\right\}=1 .
$$

This follows from the fact that density matrix of open system is normalized, $\operatorname{Tr} \widehat{\rho}_{\mathrm{s}}\left(t_{p}\right)=1$, and that the partial trace over the environment involves the vacuum projection in the tape model, eq. (31). Let us remind that we have derived the trace formula eq. (31) from the formal requirement that all the commutators are conserved, see sections VA and VC. However later, in sec. VIIIB, we find a physical interpretation of this relation: partial trace eq. (31) and the normalization (45) can be interpreted as a result of vacuum fluctuations of the classical background of the environment.

\section{Definition of the relevant density matrix}

Here we define the relevant density matrix $\widehat{\rho}_{\text {rel }}\left(t_{p}\right)$, and in the next section we discuss how it can be computed. In order to introduce the relevant density matrix $\widehat{\rho}_{\text {rel }}\left(t_{p}\right)$, we first switch to the frame of incoming/relevant/outgoing modes in the definition (43) of $\widehat{\rho}_{\text {st }}\left(t_{p}\right)$, Fig. 20 a). This change of frame was described in sec. VE1. After that all the outgoing modes which have emerged by the time moment $t_{p}$ are projected to vacuum, Fig. $20 \mathrm{~b}$ ) :

$$
\begin{aligned}
& \widehat{\rho}_{\text {rel }}\left(t_{p}\right)={ }_{\operatorname{out}(p)}\langle 0|\left\{e^{\sum_{r s=0}^{p-1} \widehat{\psi}_{r}^{\dagger} M_{r s} \widehat{\psi}_{s}}\right. \\
&\left.\times:\left|\Phi\left(t_{p}\right)\right\rangle_{\text {st st }}\left\langle\Phi\left(t_{p}\right)\right|:\right\}_{A}|0\rangle_{\operatorname{out}(p)},
\end{aligned}
$$

where

$$
|0\rangle_{\text {out }(p)}=|0\rangle_{\phi_{\text {out }}(p-1)} \otimes \ldots \otimes|0\rangle_{\phi_{\text {out }}(m)}
$$

is the joint vacuum of the outgoing modes $\phi_{\text {out }}(m), \ldots, \phi_{\text {out }}(p-1)$ which have emerged by the time moment $t_{p}$. The operation ${ }_{\operatorname{out}(p)}\langle 0|\cdot| 0\rangle_{\text {out }(p)}$ in eq. (46) denotes the projection of density matrix to the subspace of zero quanta in the outgoing modes $\phi_{\text {out }}(m), \ldots, \phi_{\text {out }}(p-1)$. This operation does not affect the other degrees of freedom. Actually this projection restricts the growing Fock space $\mathcal{F}_{\mathrm{t}}(p)$ to the subspace $\mathcal{F}_{\text {rel }}$ spanned by the relevant modes $\phi_{1} \ldots \phi_{m}$. The reduced density matrix for the open system is obtained via the projection

$$
\widehat{\rho}_{\mathrm{s}}\left(t_{p}\right)={ }_{\text {rel }}\left\langle 0\left|\hat{\rho}_{\text {rel }}\left(t_{p}\right)\right| 0\right\rangle_{\text {rel }},
$$

where here and below under $|0\rangle_{\text {rel }}$ we designate the joint vacuum for the relevant modes $\widehat{\phi}_{1}^{\dagger}, \ldots, \widehat{\phi}_{m}^{\dagger}$.

\section{Propagation of density matrix}

Given an environment with a spectral density $J(\omega)$, one computes its memory function $M\left(t-t^{\prime}\right)$. Then the incoming/outgoing/relevant modes, and disentanglers $W_{p}$ are found according to the algorithm of sec. IV D. This needs to be done once for a given $J(\omega)$.

The initial condition for the propagation is $\widehat{\rho}_{\text {rel }}(0)=$ $\left|\phi_{0}\right\rangle_{\mathrm{ss}}\left\langle\phi_{0}\right|$, since there is no relevant modes at $t_{0}=0$.

Now suppose we have $\widehat{\rho}_{\text {rel }}\left(t_{p}\right)$ which is an operator acting in $\mathcal{H}_{\mathrm{s}} \otimes \mathcal{F}_{\text {rel }}$, Fig. 21, a). The first step of propagation is the entangling step, Fig. 21, b). Here the Hamiltonian $\widehat{H}_{\text {st }}\left(\tau_{p}\right)$ of eq. (34) is applied. We use the following three facts. First, since $\widehat{H}_{\text {st }}\left(\tau_{p}\right)$ is not Hermitean, the bra state ${ }_{\text {st }}\left\langle\Phi\left(t_{p}\right)\right|$ evolves under $\widehat{H}_{\mathrm{st}}^{\dagger}\left(\tau_{p}\right)$, whereas the ket state $\left|\Phi\left(t_{p}\right)\right\rangle_{\text {st }}$ evolves under $\widehat{H}_{\text {st }}\left(\tau_{p}\right)$. Second, the Hamiltonian $\widehat{H}_{\text {st }}\left(\tau_{p}\right)$ can be commuted to the left of the pairing function in eq. (46). Analogously, the Hamiltonian $\widehat{H}_{\mathrm{st}}^{\dagger}\left(\tau_{p}\right)$ can be commuted to the right of the pairing function. Third, the Hamiltonian $\widehat{H}_{\text {st }}\left(\tau_{p}\right)$ entangles one additional incoming mode $\widehat{\psi}_{p}^{\dagger}$. Therefore, the Fock space is enlarged from $\mathcal{F}_{\text {rel }}$ to $\mathcal{F}_{\text {rel }+\mathrm{i}}$ due to the states generated by $\widehat{\psi}_{p}^{\dagger}$. The the density matrix $\widehat{\rho}_{\text {rel }}\left(t_{p}\right)$ is embedded into the space $\mathcal{H}_{s} \otimes \mathcal{F}_{\text {rel }+\mathrm{i}}$ as $\widehat{\rho}_{\text {rel }+\mathrm{i}}\left(t_{p}\right)=\widehat{\rho}_{\text {rel }}\left(t_{p}\right) \otimes|0\rangle_{\varphi_{\text {in }}(p) \varphi_{\text {in }}(p)}\langle 0|$. It propagates as

$$
\begin{aligned}
& \widehat{\rho}_{\text {rel }+\mathrm{i}}^{(1)}\left(t_{p+1}\right)=\widehat{\rho}_{\text {rel }+\mathrm{i}}\left(t_{p}\right) \\
& -i d t\left\{\widehat{H}_{\mathrm{st}}\left(\tau_{p}\right) \widehat{\rho}_{\text {rel }+\mathrm{i}}^{(1 / 2)}\left(t_{p}\right)-\widehat{\rho}_{\text {rel }+\mathrm{i}}^{(1 / 2)}\left(t_{p}\right) \widehat{H}_{\mathrm{st}}^{\dagger}\left(\tau_{p}\right)\right\},
\end{aligned}
$$



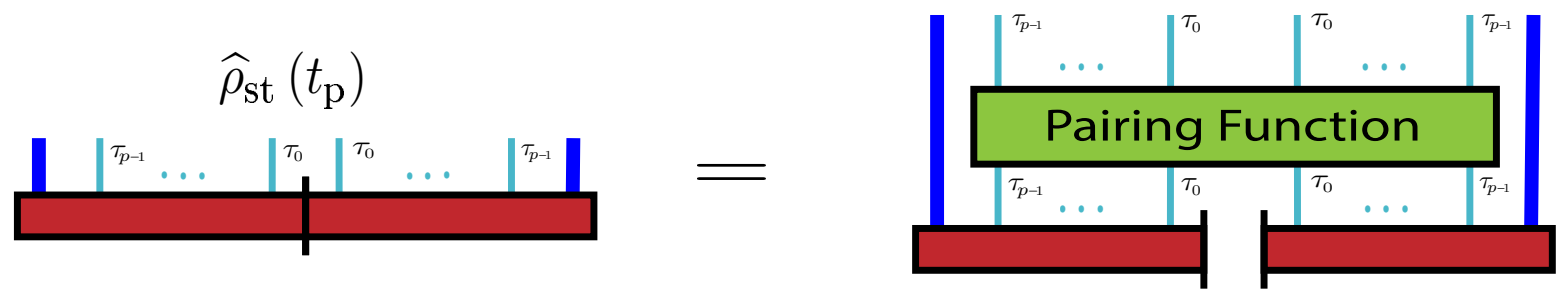

Figure 19. The joint density matrix $\widehat{\rho}_{\text {st }}\left(t_{p}\right)$ for the open system (blue lines) and the time cells (turquoise lines) is defined by applying the pairing function to $\left|\Phi\left(t_{p}\right)\right\rangle_{\text {st st }}\left\langle\Phi\left(t_{p}\right)\right|$.
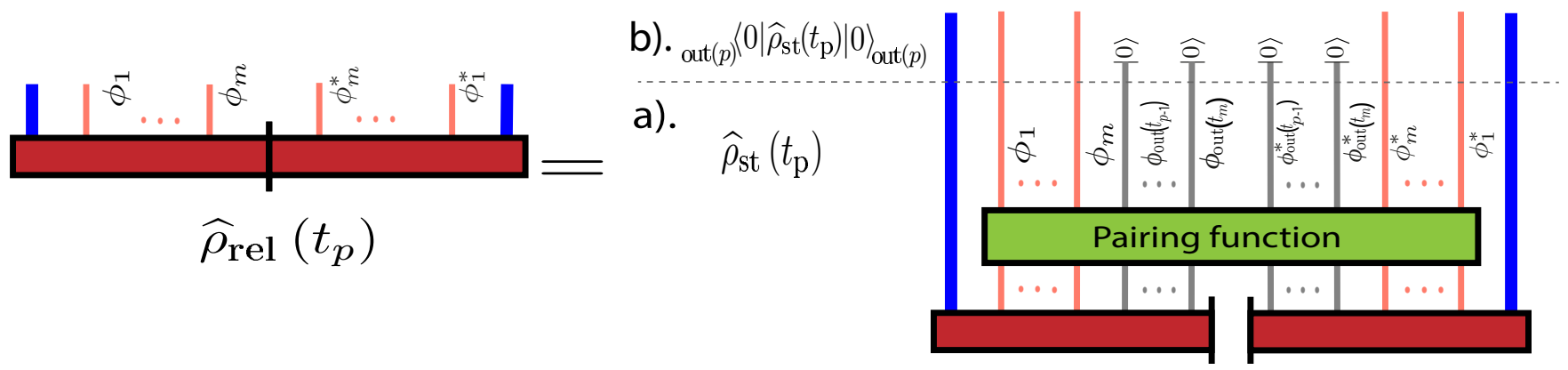

Figure 20. Renormalization group method amounts to tracing out the the irrelevant degrees of freedom as they emerge during the flow. This results in the density matrix $\widehat{\rho}_{\text {rel }}$ which is the joint reduced state of relevant degrees of freedom and of open system. This matrix is defined in two steps: a). we take the joint density matrix of open system and time cells $\widehat{\rho}_{\text {st }}\left(t_{p}\right)$ in the frame of relevant/outgoing modes; b). then we project to the vacuum all the outgoing modes which have emerged by the time moment $t_{p}$.

where $\widehat{\rho}_{\text {rel }+\mathrm{i}}^{(1 / 2)}\left(t_{p}\right)=\frac{1}{2}\left(\widehat{\rho}_{\text {rel }+\mathrm{i}}^{(1)}\left(t_{p+1}\right)+\widehat{\rho}_{\mathrm{rel}+\mathrm{i}}\left(t_{p}\right)\right)$. This propagation corresponds to the operators $\widehat{U}_{p}$ and $\widehat{U}_{p}^{\dagger}$ on Fig. 21.

Observe that $\widehat{\rho}_{\text {rel }+\mathrm{i}}^{(1)}\left(t_{p+1}\right)$ entangles one additional incoming mode $\widehat{\psi}_{p}^{\dagger}$, and the pairing function in eq. (46) does not yet contain the pairings for this mode. As follows from Fig. 18 and section VE, only the pairings of incoming mode with itself and with the relevent modes should be included. Therefore, the second step is the pairing update, Fig. $21 \mathrm{c}$ ).,

$$
\begin{array}{r}
\widehat{\rho}_{\mathrm{rel}+\mathrm{i}}^{(2)}\left(t_{p+1}\right)=\left\{e^{\widehat{\psi}_{p}^{\dagger} M(0) \widehat{\psi}_{p}+\left(\widehat{\psi}_{p}^{\dagger} \sum_{i} M_{i}(p) \widehat{\phi}_{i}+\text { c.c. }\right)}\right. \\
\left.: \widehat{\rho}_{\mathrm{rel}+\mathrm{i}}^{(1)}\left(t_{p+1}\right):\right\}_{A} .
\end{array}
$$

During the propagation the pairing update is the only operation which can make the density matrix impure. This is the non-Markovian analog of the jump $L \hat{\rho} L^{\dagger}$ terms in the Markovian Lindblad dissipator [30, 66].

In the third step of propagation, the disentangling step, Fig. $21 \mathrm{~d}$ )., we apply the disentangler $\widehat{W}_{p}$ to convert the incoming mode $\widehat{\psi}_{p}$ into the new outgoing mode $\widehat{\phi}_{\text {out }}(p)$ :

$$
\widehat{\rho}_{\mathrm{rel}+\mathrm{o}}^{(3)}\left(t_{p+1}\right)=\widehat{W}_{p} \widehat{\rho}_{\mathrm{rel}+\mathrm{i}}^{(2)}\left(t_{p+1}\right) \widehat{W}_{p}^{-1} .
$$

Here we change the subscript from 'rel $+\mathrm{i}$ ' to 'rel $+\mathrm{o}$ ' in order to make explicit the fact that (i) we start with the
Fock space spanned by the relevant modes $\widehat{\phi}_{1}^{\dagger} \ldots \widehat{\phi}_{m}^{\dagger}$ and one incoming mode $\widehat{\psi}_{p}^{\dagger}$, and (ii) after the disentangler transform we end with the Fock space spanned by the relevant modes $\widehat{\phi}_{1}^{\dagger} \ldots \widehat{\phi}_{m}^{\dagger}$ and one outgoing mode $\widehat{\phi}_{\text {out }}^{\dagger}(p)$.

The last step, the oblivion, $21 \mathrm{e}$ )., is to discard the quantum content of this irrelevant mode:

$$
\widehat{\rho}_{\text {rel }}\left(t_{p+1}\right)=_{\phi_{\text {out }}\left(t_{p}\right)}\left\langle 0\left|\widehat{\rho}_{\text {rel }+\mathrm{o}}^{(3)}\left(t_{p+1}\right)\right| 0\right\rangle_{\phi_{\text {out }}\left(t_{p}\right)} .
$$

As a result, we return to the smaller Fock space $\mathcal{F}_{\text {rel }}$. Then the propagation procedure is repeated for the next time moment $t_{p+1}$.

These four steps, eqs. (49), (50), (51), (52), constute one complete iteration of the real-time RG flow. They can be implemented numerically in a Fock space of the tape cells (see Fig 22), see appendix A. which is truncated in the maximal total occupation of the modes. The pairing update (50) is inexpensive since we can truncate it to second order, see appendix B. The disentangler (52) can be efficiently implemeted using Lanczos-like algorithms: see appendix C.

\section{E. Example calculation}

In Fig. 23 we present the results of calculation of the RG for density matrices for the system of a driven qubit coupled to the waveguide eq. (11) with $\varepsilon=1$ and 


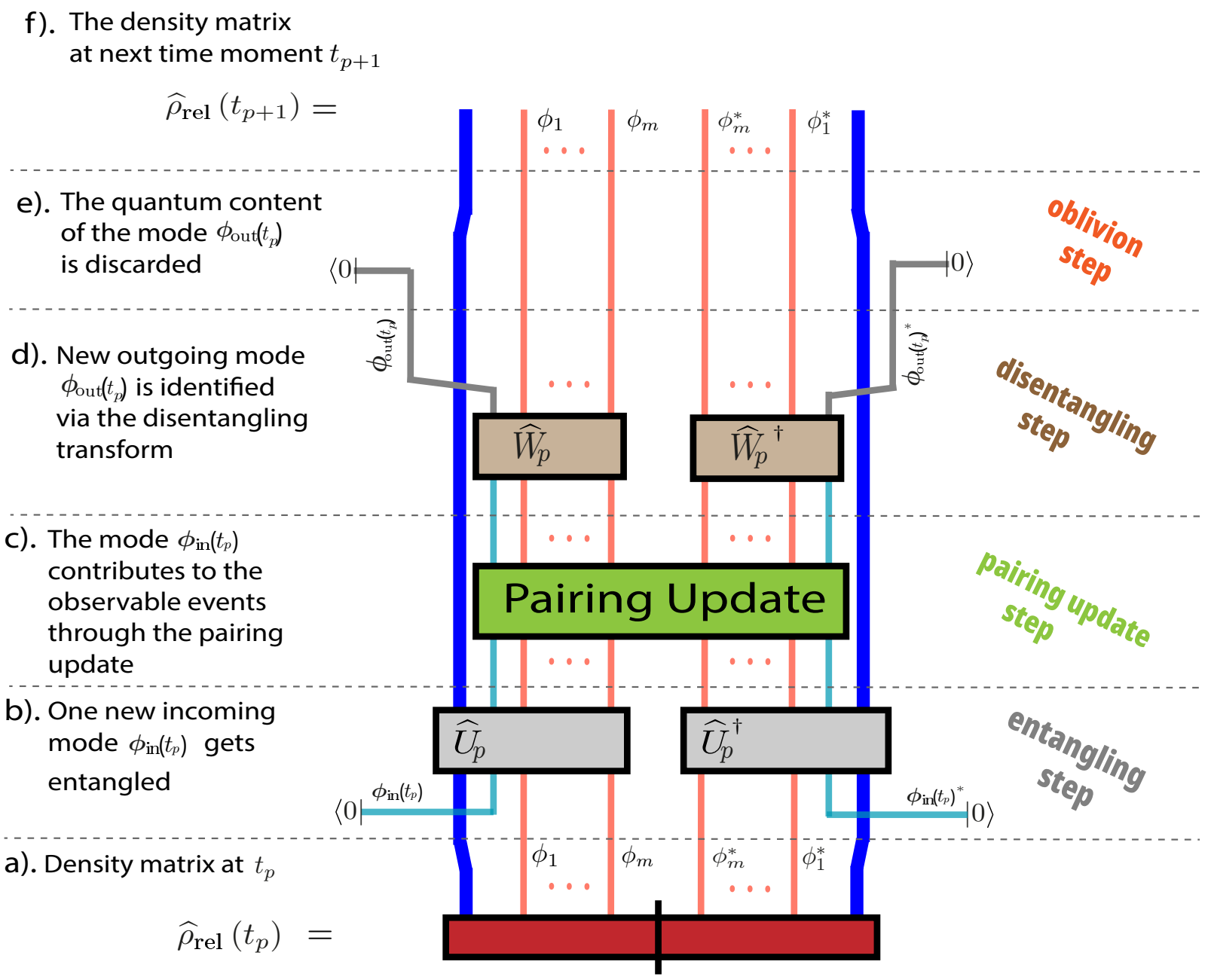

Figure 21. The lifecycle of entanglement in course of real time evolution. It directly maps to the numerical recipe of the renormalization group for density matrices.

$h=0.05$. We have: $\widehat{H}_{\mathrm{s}}=\widehat{\sigma}_{+} \widehat{\sigma}_{-}+0.1 \cos t$, and $\widehat{s}=\widehat{\sigma}_{-}$. We conduct a series of simulations with one $(m=1)$, two $(m=2)$, three $(m=3)$, and five $(m=5)$ relevant modes. The convergence is achieved when only the states of no more than two quanta were kept in the tape Fock spaces $\mathcal{F}_{\mathrm{t}}(p), \mathcal{F}_{\text {rel }}, \mathcal{F}_{\text {rel }+\mathrm{i}}, \mathcal{F}_{\text {rel }+\mathrm{o}}$. It is seen that the simulation converges already for the three $(m=3)$ relevant modes. In Fig. 24 we compare the converged result with the numerically-exact solution of the Schrodinger equation. The Schrodinger equation was solved in the truncated Hilbert space of the model (11). The truncation was done by keeping the first 14 lattice sites $\widehat{a}_{1}^{\dagger} \ldots \widehat{a}_{14}^{\dagger}$ and by keeping all the Fock states $\widehat{a}_{1}^{\dagger n_{1}} \ldots \widehat{a}_{14}^{\dagger n_{14}}|0\rangle_{\mathrm{b}}$ with maximal total occupation $n_{1}+\ldots+n_{14} \leq 7$. Our RG procedure yields a benefit in comparison with the "bare" solution of the Schrodinger equation: 3 modes vs 14 and 2 quanta vs 7 . The physical explanation is that among the 14 modes only 3 are non-neglibigly coupled to the future motion, and their occupation is rather small, so that 2 quanta are enough.

\section{F. Balance of complexity}

As we discussed in the Introduction, the quantum complexity is expected to saturate if the rates of occurences of incoming and outgoing modes are equal, and the incoing and outgoing fluxes are also equal. Here we discuss there questions on the example of the driven qubit in the waveguide from the previous section.

\section{Rates of occurences of incoming and ougoing modes}

As it is seen from the Fig. 22, the rates of ougoing modes are not always equal to the rates of incoming modes. On the time interval $\left[0, t_{m}\right]$ every incoming mode becomes relevant: no outgoing modes are produced at all. During this initial period the dimension of the relevant space grows linearly with time. However, due to the fastest decoupling property, Fig. 7, when the dimension becomes equal to $m$, the contribution of new relevant 


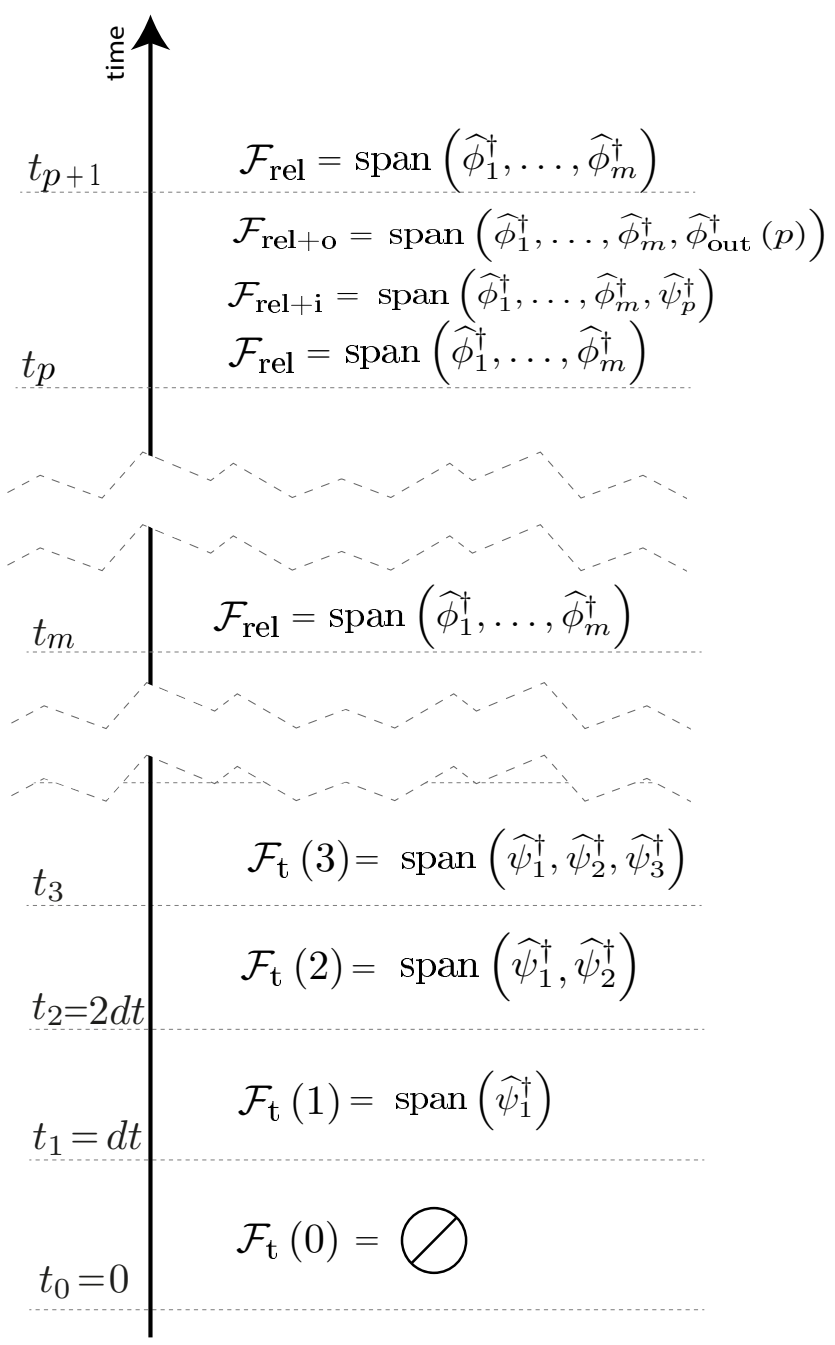

Figure 22. The renormalization group for density matrices has two regimes. 1). Before the time moment $t_{m}$ all the time cells are relevant, and the tape Fock space continuously grows. 2 ). After the time moment $t_{m}$ the Fock space stops growing and starts to "oscillate" between $\mathcal{F}_{\text {rel }}, \mathcal{F}_{\text {rel }+\mathrm{i}}, \mathcal{F}_{\text {rel }+ \text { o }}$. Here span (...) designates the Fock space generated by applying creation operators inside the brackets to the vacuum state. In a numerical simulation the Fock spaces are truncated in the maximal total occupation of the modes. Such a truncation is justified by the conjectured balance of complexity: see sec. VIF. In a numerical simulation the Fock spaces $\mathcal{F}_{\text {rel }+\mathrm{i}}$ and $\mathcal{F}_{\text {rel+o }}$ are the same: it is our interpretation that the additional mode alternatively plays the role of the incoming and outgoing mode.

modes becomes exponentially small. The relevant space saturates. We can keep the dimension fixed to $m$ and start to produce the outgoing modes with the rate equal to the rate of incoming modes.

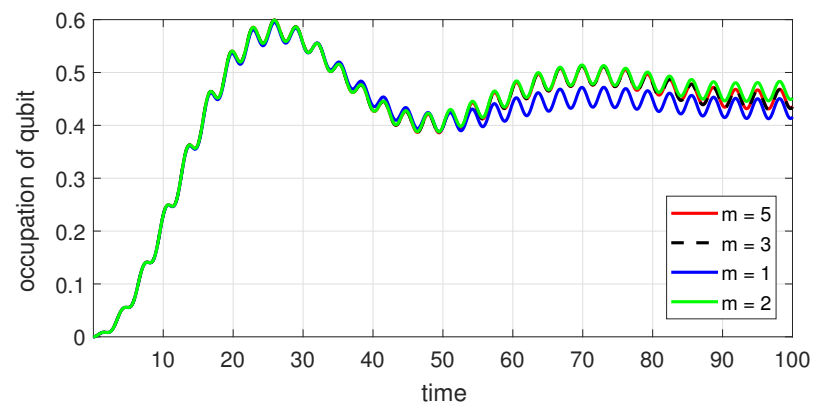

Figure 23. For the system of a driven qubit in a highly nonMarkovian waveguide, only 3 relevant modes at a maximal occupation of 2 quanta is enough to achieve the convergence of observable properties. The calculation is done by RG for density matrices.

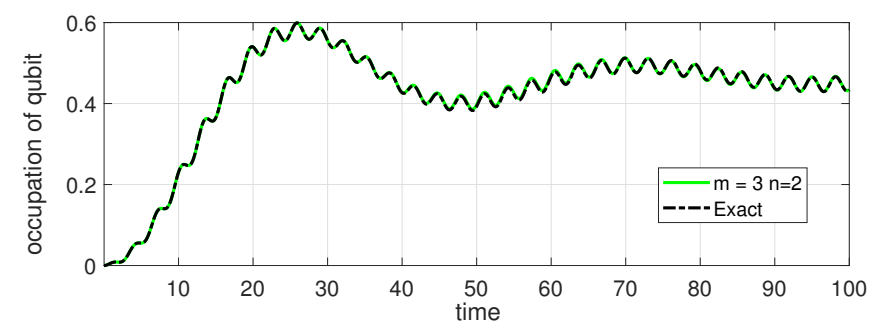

Figure 24. For the same situation as in previous Fig. 23, we compare the converged RG for density matrices with the numerically exact solution of Schrodinger equation. The latter required us to keep 14 lattice sites in eq. (11) and all states of maximum total occupation of 7 quanta.

\section{Balance of fluxes}

For the same system of a driven qubit in the waveguide, we check the proposed conjecture that the flux of the quanta emitted into the incoming mode (the incoming current) and the flux of the quanta discarded in the ougoing mode (the outgoing current) should balance each other. The results of the Fig. 25 support our conjecture. The incoming current $j_{\text {in }}\left(t_{p}\right)$ is computed as the population of the incoming mode $\varphi_{\text {in }}(p)$ at the end of stage b) in Fig. 21 (after propagation under $\widehat{U}_{p} \ldots \widehat{U}_{p}^{\dagger}$, but before the pairing update):

$$
j_{\text {in }}\left(t_{p}\right)=\frac{1}{d t} \operatorname{Tr}\left\{\widehat{\rho}_{\mathrm{rel}+\mathrm{i}}^{(1)}\left(t_{p+1}\right) \widehat{\psi}_{p}^{\dagger} \widehat{\psi}_{p}\right\}
$$

The outgoing current $j_{\text {out }}\left(t_{p}\right)$ is computed as the population of the outgoing mode $\phi_{\text {out }}(p)$ at the end of stage d) on Fig. 21 (after disentangling under $\widehat{W}_{p} \ldots \widehat{W}_{p}^{\dagger}$ but before the oblivion):

$$
j_{\text {out }}\left(t_{p}\right)=\frac{1}{d t} \operatorname{Tr}\left\{\widehat{\rho}_{\text {rel }+ \text { o }}^{(3)}\left(t_{p+1}\right) \widehat{\phi}_{\text {out }}^{\dagger}(p) \widehat{\phi}_{\text {out }}(p)\right\} .
$$



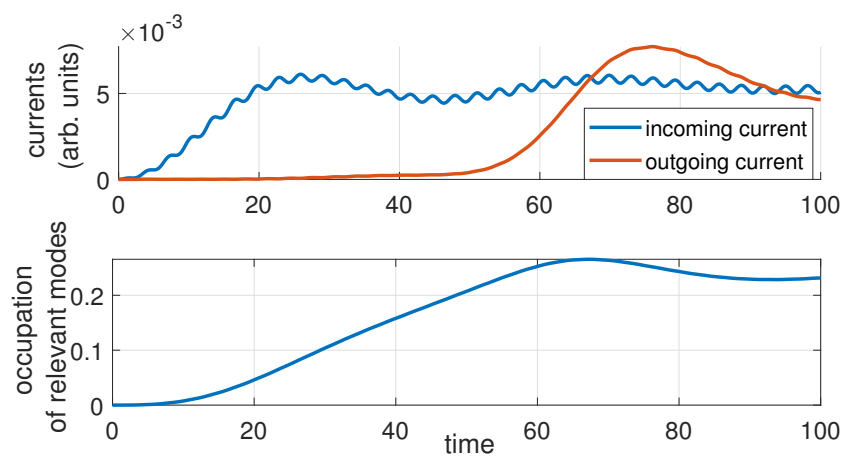

Figure 25. The system of a driven qubit in a waveguide. The outgoing current of quanta which are discarded in the outgoing modes balances the incoming flux of quanta which are emitted into the incoming modes. The units for the currents are common but otherwise arbitrary. As a consequence, the total occupation of the relevant modes saturates.

Finally, the total occupation of relevant modes is computed as

$$
n_{\text {tot }}\left(t_{p}\right)=\operatorname{Tr}\left\{\widehat{\rho}_{\text {rel }+\mathrm{i}}^{(1)}\left(t_{p+1}\right)\left(\widehat{\psi}_{p}^{\dagger} \widehat{\psi}_{p}+\sum_{k=1}^{m} \widehat{\phi}_{k}^{\dagger} \widehat{\phi}_{k}\right)\right\} .
$$

This balance of compelxity has the following physical interpretation, Fig. 26. The incoming modes inject new quanta with the flux $j_{\text {in }}\left(t_{p}\right)$ into the "cavity" which is formed by the relevant modes. The "mirrors" of the cavity are formed by the coupling to the future motion. These "mirrors" are imperfect: the outgoing modes are continuously leaking out and carry away with them a flux $j_{\text {out }}\left(t_{p}\right)$ of quanta.

\section{G. Analogies with the time-symmetric formulation of quantum mechanics}

Observe that the general structure of the RG flow described above is time-symmetric: if we apply the steps in Fig. 21 forward in time, then i) one incoming mode in the vacuum gets entangled; ii) one outgoing mode gets disentangled and projected to the vacuum. If we reverse this procedure in time, we get: i') one outgoing mode in the vacuum gets entangled; ii') one incoming mode gets disentangled and projected to the vacuum. This has interesting analogies to the two-state vector formulation (TSVF) of quantum mechanics by Yakir Aharonov et al. [67]. In TSVF one considers the two measurements. The outcome of the first measurement at time $t$ produces the state $\psi_{\text {in }}(t)$. The outcome of the second measurement at $T>t$ produces the state $\psi_{\text {out }}(T)$. Then TSVF considers the quantum evolution inside the interval $[t, T]$ with the fixed initial and final boundary conditions $\psi_{\text {in }}(t)$ and $\psi_{\text {out }}(T)$ correspondingly, Fig. 27 This formalism is applied when analysing the experiments with the postselection of measurement results [68]. This formalism also yields an interpretation of quantum mechanics: one may assume that there is a final boundary condition for the Universe which singles out a definite outcome for all the measurements [69]. However the real-time entanglement structure suggests an interesting alternative: instead of global initial and final states, one may consider the streams of initial and final states, so that there is a continuous competition between the birth and the death of the quantum reality in real time, see Fig. 27

\section{MARKOVIAN LIMIT}

In this section we relate our renormalization group procedure Fig. 21 to the Lindblad master equation which is applied in the Markovian regime [66, 70]. This will help us to develop intuition about the steps of our RG procedure.

There are many ways to perform the Markovian limit. A good way should be based solely on the spectral decoupling mechanism represented in sec. IV. A rough way is to apply the conventional formal arguments which lead to the Lindblad master equation. Here we choose the latter in order to connect the RG procedure to the conventional terms. Moreover, later it will help us to assess the additional insights provided by the spectral decoupling mechanism.

We choose the derivation of Markovian limit via the quantum stochastic differential equations [66, 70]. Essentially this approach assumes that we are dealing with a small-coupling, near-resonant, and broadband setup. Then the time cells can be considered as local independent degrees of freedom, $\left[\widehat{b}(t), \widehat{b}^{\dagger}\left(t^{\prime}\right)\right]=\Gamma \delta\left(t-t^{\prime}\right)$. In terms of the stream of discrete time moments this reads

$$
\left[\widehat{b}\left(\tau_{p}\right), \widehat{b}^{\dagger}\left(\tau_{q}\right)\right]=d t^{-1} \Gamma \delta_{p q}
$$

This means that we have $m=0$ relevant modes: only incoming mode appears in Fig. 21. The disentangler in Fig. $21, \mathrm{~d})$ is the identity operator: $\phi_{\text {out }}(p)=\phi_{\text {in }}(p)$. The small-coupling assumption means that we can consider at most one quantum in the incoming mode.

The stage a) in Fig. 21 considers the relevant density matrix $\widehat{\rho}_{\text {rel }}\left(t_{p}\right)$ at a time moment $t_{p}$, which is simply

$$
\widehat{\rho}_{\text {rel }}\left(t_{p}\right)=\widehat{\rho}_{\mathrm{s}}\left(t_{p}\right)
$$

where $\widehat{\rho}_{\mathrm{S}}\left(t_{p}\right)$ is a reduced density matrix of open system.

Let us assume for a moment that $\widehat{\rho}_{\text {rel }}\left(t_{p}\right)$ is a pure state $|\phi\rangle_{\text {rel }}$. After the entangling step, Fig. $21 \mathrm{~b}$ )., this state becomes

$$
\begin{aligned}
\left|\phi^{(1)}\right\rangle_{\mathrm{rel}+\mathrm{i}}= & \left(1-i \widehat{H}_{\mathrm{s}} d t\right)|\phi\rangle_{\mathrm{rel}} \otimes|0\rangle_{\varphi_{\mathrm{in}}(p)} \\
& -i \widehat{s}|\phi\rangle_{\mathrm{rel}} \otimes|1\rangle_{\varphi_{\mathrm{in}}(p)} d t \\
& -\frac{1}{2} \Gamma \widehat{s}^{\dagger} \widehat{s}|\phi\rangle_{\mathrm{rel}} \otimes|0\rangle_{\varphi_{\mathrm{in}}(p)} d t
\end{aligned}
$$


b).

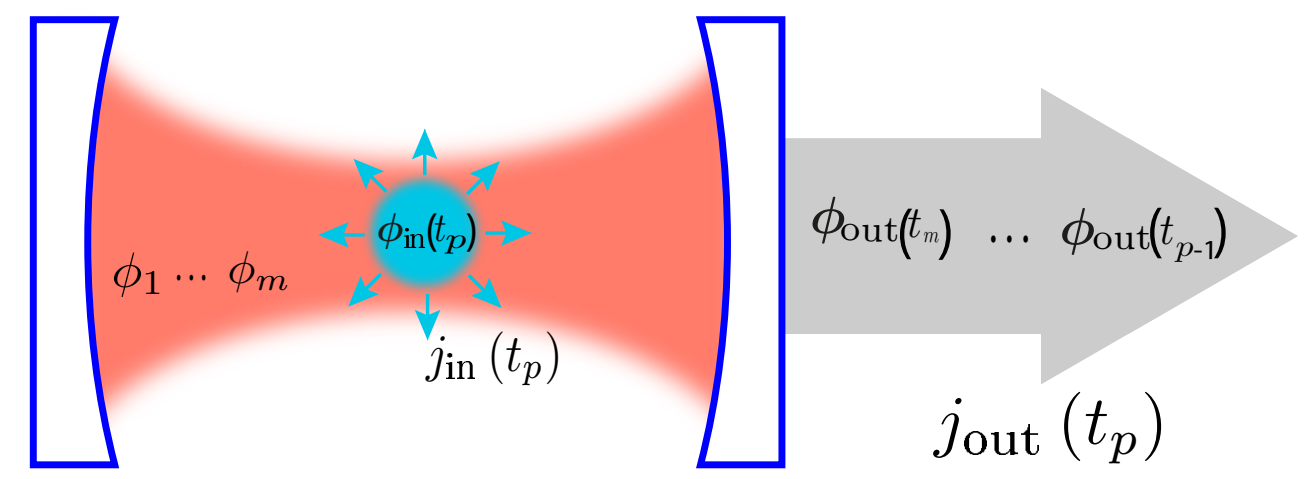

a).

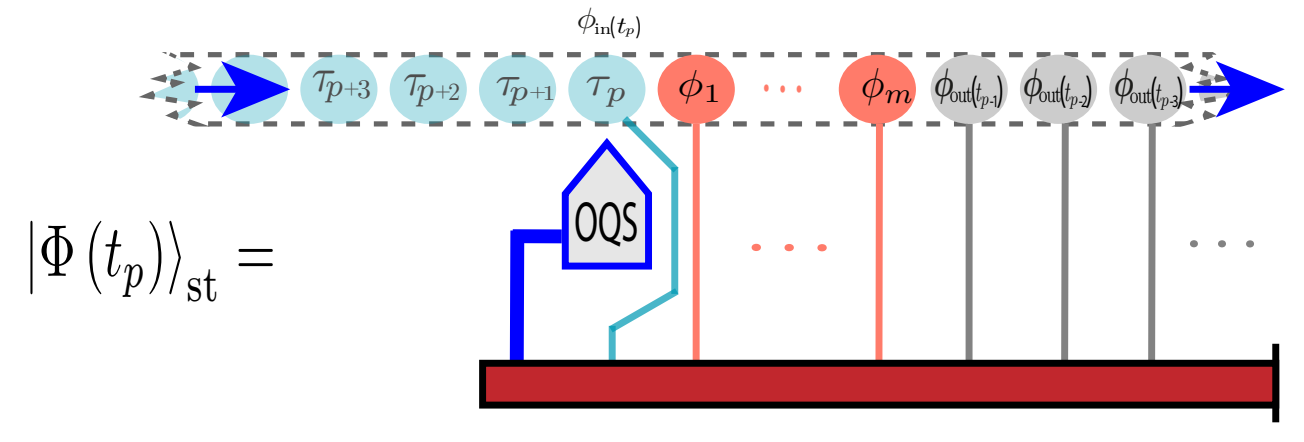

Figure 26. We conjecture that the quantum complexity of the real-time motion of open quantum systems is asymptotically bounded. The interpretation is the following a). Suppose we are given a joint quantum state $\left|\Phi\left(t_{p}\right)\right\rangle_{\text {st }}$ for the open system and the time cells, in the frame of incoming/relevant/outgoing modes. b). It can be interpreted that the relevant modes $\phi_{1} \ldots \phi_{m}$ play the role of the "cavity" modes. The "mirrors" of the cavity are the coupling to the future evolution. New quanta are injected into the "cavity" with a flux $j_{\text {in }}\left(t_{p}\right)$ via the incoming mode $\varphi_{\text {in }}(p)$. However the "mirrors" are imperfect: the outgoing modes are continuously leaking out and carry away with them a flux $j_{\text {out }}\left(t_{p}\right)$ of quanta. As a result, the total occupation $n_{\text {tot }}\left(t_{p}\right)$ of the "cavity" modes is expected to be bounded due to the balance of the fluxes.

Here we keep only $O(d t)$ terms in the midpoint propagation under (34). In the last line we see the secondorder virtual process because there is $d t^{-1}$ in the memory function commutator (56). We observe that the first line yields the unitary free motion of open system $-i\left[\widehat{H}_{\mathrm{s}}, \widehat{\rho}_{\text {rel }}\right]$ in the Lindblad equation, and the last line yields the standard term $-\frac{1}{2} \Gamma\left\{\widehat{s}^{\dagger} \widehat{s}, \widehat{\rho}_{\text {rel }}\right\}$ which describes the decay of probability of no-emission event.

After applying the pairing update, Fig. 21 c)., to the state $\left|\phi^{(1)}\right\rangle_{\text {rel+i }}$, we obtain the quantum jump term from the second line of eq. (58):

$$
\begin{aligned}
& \widehat{\rho}_{\text {rel }+\mathrm{i}}^{(2)}\left(t_{p}\right)=\left(\widehat{\rho}_{\text {rel }}-i\left[\widehat{H}_{\mathrm{s}}, \widehat{\rho}_{\text {rel }}\right] d t\right) \otimes|0\rangle_{\varphi_{\text {in }}(p) \varphi_{\text {in }}(p)}\langle 0| \\
& -\frac{1}{2} \Gamma\left\{\widehat{s}^{\dagger} \widehat{s}, \widehat{\rho}_{\mathrm{rel}}\right\} d t \otimes|0\rangle_{\varphi_{\mathrm{in}}(p) \varphi_{\mathrm{in}}(p)}\langle 0| \\
& +\Gamma \widehat{s} \widehat{\rho}_{\text {rel }} \widehat{s}^{\dagger} d t \otimes|0\rangle_{\varphi_{\text {in }}(p) \varphi_{\text {in }}(p)}\langle 0| \\
& +\left\{\text { terms containing either }|1\rangle_{\varphi_{\mathrm{in}}(p)} \text { or }{ }_{\varphi_{\mathrm{in}}(p)}\langle 1|\right\} \text {. }
\end{aligned}
$$

Finally, the oblivion step, Fig. 21 e)., removes the emitted quanta and the incoming mode. We obtain the conventional Lindblad equation:

$$
\begin{aligned}
& \widehat{\rho}_{\mathrm{s}}\left(t_{p+1}\right)=\widehat{\rho}_{\mathrm{s}}\left(t_{p}\right) \\
& \quad-i\left[\widehat{H}_{\mathrm{s}}, \widehat{\rho}_{\mathrm{s}}\right] d t-\frac{1}{2} \Gamma\left\{\widehat{s}^{\dagger} \widehat{s}, \widehat{\rho}_{\mathrm{s}}\right\} d t+\Gamma \widehat{s} \widehat{\rho}_{\mathrm{s}} \widehat{s}^{\dagger} d t .
\end{aligned}
$$

Therefore, we obtain the following "rough" picture of the Markovian mode. Each infinitesimal time interval 

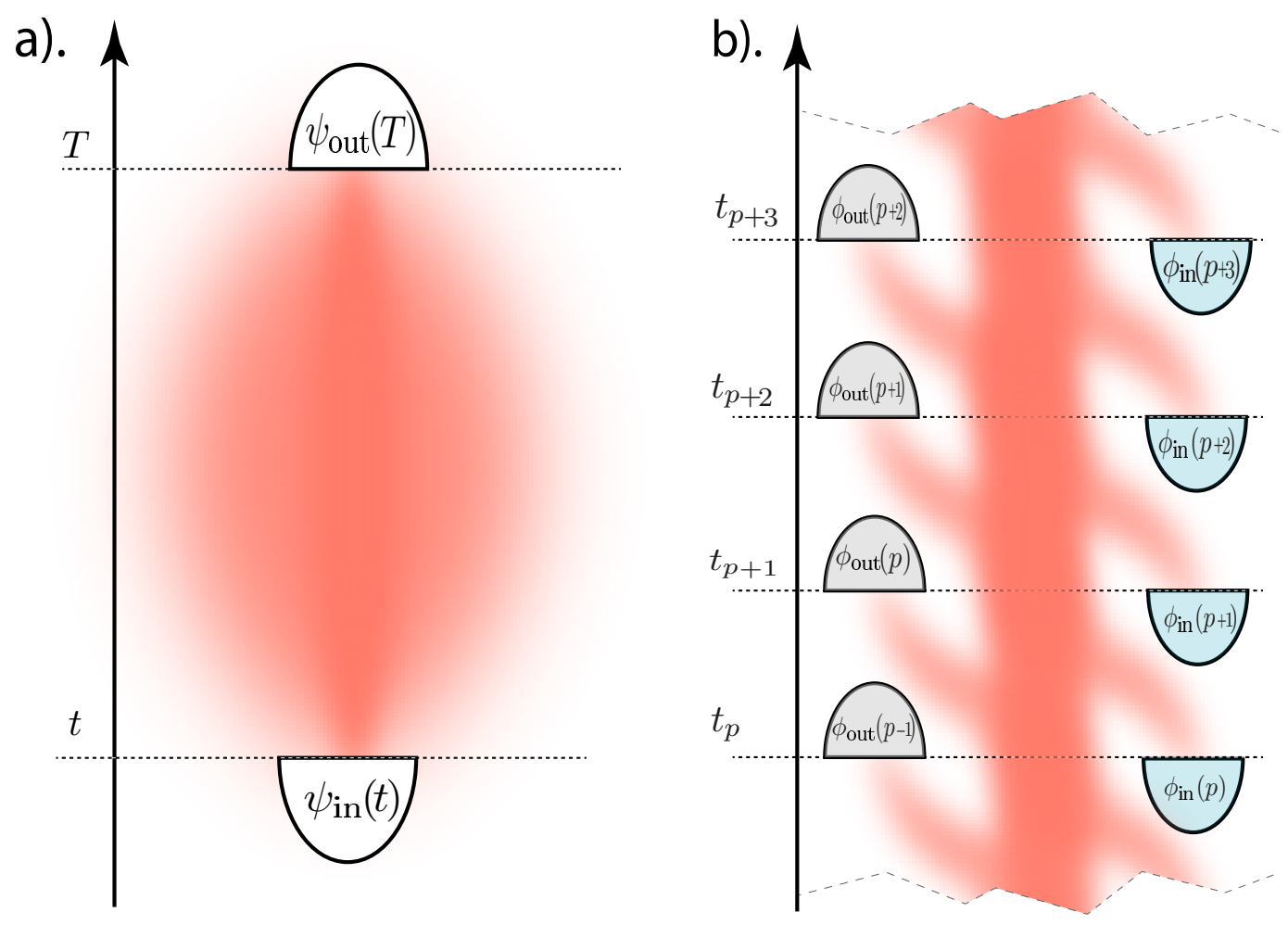

Figure 27. a). In the time-symmetric formulation of quantum mechanics (also called the two-state vector formalism, TSVF) of Yakir Aharonov et al. [67], the quantum evolution is considered which starts from some initial state $\psi_{\text {in }}(t)$. Then one assumes that there exists a final boundary condition in the future $\psi_{\text {out }}(T)$. The conjecture is that this final boundary condition is "fine-tuned" to yield an effective collapse to definite outcomes of all the quantum measurements happening inside the interval $[0, T][69]$. b). However the real-time structure of entanglement suggests an interesting alternative: instead of the speculated global future boundary condition, there are streams of initial $\varphi_{\text {in }}(p)$ and final $\varphi_{\text {out }}(p)$ states, so that there is a continuous competition between the birth and the death of the quantum reality during each infinitesimal time interval.

one incoming mode $\varphi_{\text {in }}(p)$ is coupled to the open system; a quantum is emitted into $\varphi_{\text {in }}(p)$ during the entangling step; the quantum jump is taken into account via the pairing update. After that, the incoming mode (which is equal to the outgoing mode in this case) is irreversibly decoupled and the quantum is discarded.

In summary, our RG procedure can be considered as an entanglement-assisted non-Markovian generalization of the Lindblad master equation [30].

\section{STOCHASTIC UNRAVELLING: RENORMALIZATION GROUP ALONG A QUANTUM TRAJECTORY}

Here we implement our intuition that the outgoing modes in Fig. 1 can be replaced by a classical stochastic signal. In other words, we explore the second alternative for the irrelevant degrees of freedom: instead of tracing them out, we collapse them to a classical noise as soon as they emerge. This results in a stochastic variant of renormalization group which we call the renormalization group along a quantum trajectory. This way we com- pletely implement the intuitive picture of Introduction that the ultimate fate of the emitted field is not only to decouple but also to become observed.

\section{A. Ensembles vs quantum trajectories}

Let us recall that in the Markovian regime the motion of open quantum system can be described in the two alternative but equivalent ways [66]. The first way is the ensemble description. It is given in terms of a reduced density matrix and its evolution is governed by the Lindblad master equation. The second way is the description by the quantum trajectories. Here one takes into account the fact that every open system will exercise a random motion under the influence of the environment. The environment stores a classical record of the history of such a motion. The quantum trajectory is the quantum evolution which is conditioned on a particular realization of the record. This results in a stochastic pure-state evolution which is known under various names in literature: quantum jumps, quantum state diffusion, stochas- 
tic wavefunction etc. Then the description in terms of the reduced density matrix is recovered as average over the ensemle of all the possible records (hence the name "ensemble description"). One also says about this in other words: that the quantum trajectories provide a stochastic unravelling of the dissipative master equations.

The merit of quantum trajectories is two-fold. First, they yield efficient Monte Carlo simulation methods for the dissipative dynamics. This is because the size of density matrix scales as $N^{2}$ with the dimension $N$ of the Hilbert space [30, 71]. While the size of a pure state of the quantum trajectory scales as $N$. Second, they provide the interpretation for the experiments on observing and manipulating a single quantum system, under the Markovian conditions [66].

Our renormalization group for density matrices can also be considered as an ensemble description. Then the question arises what is the corresponding quantum trajectory description. The merit of this is again two-fold: we obtain a). efficient Monte-Carlo simulations for pure states and b). new insights into how the classical records are stored in the non-Markovian environment.

\section{B. Pairing function as average over the vacuum fluctuations of environment}

In the Markovian regime the "sandwitch" term $\Gamma \widehat{s} \widehat{\rho}_{\mathrm{s}} \widehat{s}^{\dagger}$ arises as average over the ensemble of quantum-jump histories $[30,66,70]$. In our renormalization group the pairing function plays the role of the non-Markovian "sandwitch" term, see eq. (44) and (59). Therefore, we want to represent it as an ensemble of some observable events. Here we show that as such events one can choose the vacuum fluctuations of the classical field in the environment, see Fig. 28.

The classical field $z$ is carried by the coherent state

$$
|z\rangle_{\mathrm{b}}=\mathcal{Q}_{\mathrm{vac}}(z)^{\frac{1}{2}} \exp \left(\int d \omega z^{*}(\omega) \widehat{a}^{\dagger}(\omega)\right)|0\rangle_{\mathrm{b}},
$$

where the normalization factor $\mathcal{Q}_{\text {vac }}(z)$ is the probability to observe a vacuum fluctuation $\mathrm{z}$ of the field,

$$
\mathcal{Q}_{\text {vac }}(z)=\left.\left.\right|_{\mathrm{b}}\langle z \mid 0\rangle_{\mathrm{b}}\right|^{2} \propto \exp \left(-\int d \omega|z(\omega)|^{2}\right) .
$$

At the coupling site $\widehat{b}^{\dagger}(t)$ the vaccum fluctuation $z$ appears as a colored (non-Markovian) noise $\xi^{*}(t)$ :

$$
\xi^{*}(t)=\int d \omega c^{*}(\omega) z^{*}(\omega) e^{i \omega t} .
$$

This noise has the Gaussian statistics

$$
\overline{\xi_{r}}=\overline{\xi_{r}^{*}}=0, \overline{\xi_{r} \xi_{s}^{*}}=M_{r s},
$$

where we consider the noise $\xi$ at the midpoint times: $\xi_{r}=$ $\xi\left(\tau_{r}\right)$. Each realization of $z$ leads to a single realization of the entire time-trajectory of $\xi^{*}(t)$.
It turns out that the pairing function can be represented as an average over these vacuum fluctuations, Fig. . 28:

$$
\left\{e^{\sum_{r s=0}^{\infty} \widehat{\psi}_{r}^{\dagger} M_{r s} \widehat{\psi}_{s}}\right\}_{A}=\overline{\left[\left\{e^{\sum_{r=0}^{\infty} \xi_{r} \widehat{\psi}_{r}^{\dagger}} e^{\sum_{s=0}^{\infty} \xi_{s}^{*} \widehat{\psi}_{s}}\right\}_{A}\right]_{\xi}}
$$

where $\{\cdot\}_{A}$ is the antinormal averaging. Observe that in the pairing function we extend the summation range from $[0, p-1]$ (as in eq. (43)) to $[0, \infty)$. This is possible since the incoming modes $\phi_{\text {in }}(p), \phi_{\text {in }}(p+1), \ldots$ are empty at a time moment $t_{p}$. Then the density matrix eq. (43) is represented as average over the ensemble of pure states, Fig. 29,

$$
\widehat{\rho}_{\mathrm{st}}\left(t_{p}\right)={\overline{\left[\left|\Phi\left(\xi, t_{p}\right)\right\rangle_{\mathrm{st} \mathrm{st}}\left\langle\Phi\left(\xi, t_{p}\right)\right|\right]_{\xi}}} .
$$

Here the joint pure state of the time cells and of the open system is

$$
\left|\Phi\left(\xi, t_{p}\right)\right\rangle_{\mathrm{st}}=e^{\sum_{s=0}^{\infty} \xi_{s}^{*} \widehat{\psi}_{s}}\left|\Phi\left(t_{p}\right)\right\rangle_{\mathrm{st}} .
$$

Observe the interpretation of the state $\left|\Phi\left(\xi, t_{p}\right)\right\rangle_{\text {st }}$. The action of operator

$$
\widehat{S}(\xi)=e^{\sum_{s=0}^{\infty} \xi_{s}^{*} \widehat{\psi}_{s}}
$$

induces a non-unitary Bogoliubov transform of the creation/annihilation operators in $\left|\Phi\left(t_{p}\right)\right\rangle_{\mathrm{st}}$ :

$$
\begin{gathered}
\widehat{S}(\xi) \widehat{\psi}_{r} \widehat{S}^{-1}(\xi)=\widehat{\psi}_{r}, \\
\widehat{S}(\xi) \widehat{\psi}_{r}^{\dagger} \widehat{S}^{-1}(\xi)=\widehat{\psi}_{r}^{\dagger}+\xi_{r}^{*}
\end{gathered}
$$

In other words, the operation $\widehat{S}(\xi)$ introduces a classical background $\xi$ in the state $\left|\Phi\left(t_{p}\right)\right\rangle_{\text {st }}$, see Fig. 29. As a result, each emitted quantum $\widehat{\psi}_{r}^{\dagger}$ in $\left|\Phi\left(\xi, t_{p}\right)\right\rangle_{\text {st }}$ becomes a superposition of the classical background $\xi_{r}^{*}$ and of a purely quantum part:

$$
\widehat{\psi}_{r}^{\dagger} \rightarrow \xi_{r}^{*}+\widehat{\psi}_{r}^{\dagger}
$$

We call $\left|\Phi\left(\xi, t_{p}\right)\right\rangle_{\text {st }}$ the quantum state over a classical background $\xi$. When we perform the partial trace (42),

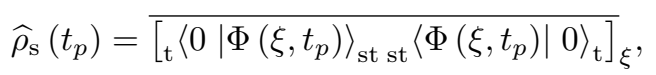

the purely quantum part gets discarded due to projection to the vacuum. In other words, all the quanta collapse to the classical background,

$$
\widehat{\psi}_{r}^{\dagger} \rightarrow \xi_{r}^{*}
$$

and then we average over all the possible vacuum fluctuations. 


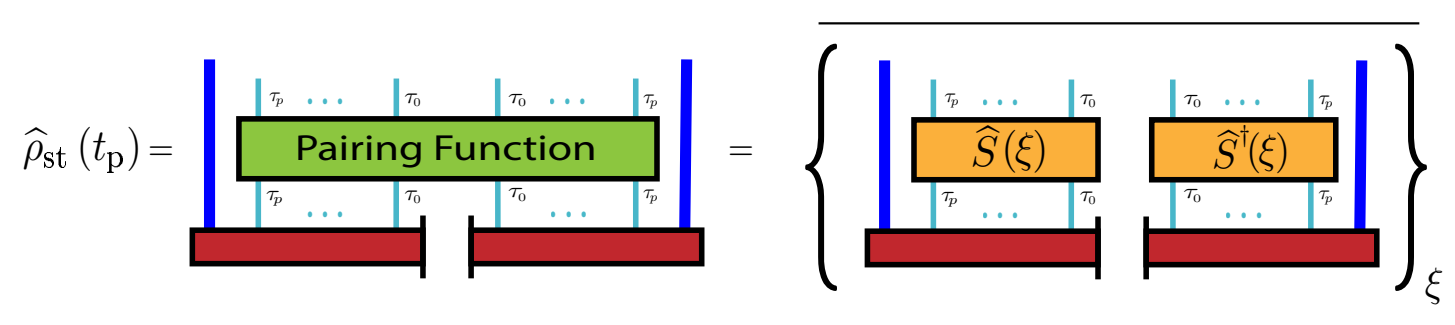

Figure 28. The pairing function can be stochastically decoupled into the two separate displacement operators $\widehat{S}(\xi)$ and $\widehat{S}^{\dagger}(\xi)$ which act on the ket and the bra states correspondingly. The averaging is over the displacement $\xi$ which is a classical noise of vacuum fluctuations at the coupling site $\widehat{b}^{\dagger}(t)$.

$$
\left|\Phi\left(\xi, t_{p}\right)\right\rangle_{\mathrm{St}}=\begin{array}{|l|l|}
\mid \tau_{p} \ldots & \tau_{0} \\
\hline \multicolumn{1}{|c|}{\widehat{S}(\xi)} \\
\hline \tau_{p} \ldots & \tau_{0} \\
\hline
\end{array}
$$

Figure 29. The density matrix $\widehat{\rho}_{\text {st }}\left(t_{p}\right)$ can be represented as the ensemble of displaced pure states $\left|\Phi\left(\xi, t_{p}\right)\right\rangle_{\text {st }}$. This state is displaced by $\widehat{S}(\xi)$. The result is that the emitted quanta are "centered" on a classical signal $\xi$, see eqs. (68), (69), (70)).

\section{Renormalization group along a quantum trajectory}

Now we derive the evolution equations in which the irrelevant degrees are collapsed to a classical noise as soon as they emerge.

Below we follow the convention of the growing Fock space of sec. VIA. Then the wavefunction $\left|\Phi\left(t_{p}\right)\right\rangle_{\text {st }}$ belongs to the space $\mathcal{H}_{\mathrm{s}} \otimes \mathcal{F}_{\mathrm{t}}(p)$, with the Fock space $\mathcal{F}_{\mathrm{t}}(p)$ being spanned by the time cells $\psi_{p-1}^{\dagger} \ldots \psi_{0}^{\dagger}$.

Let us introduce the relevant wavefunction

$$
\left|\Phi\left(\xi, t_{p}\right)\right\rangle_{\mathrm{rel}}={ }_{\operatorname{out}(p)}\left\langle 0\left|e^{\sum_{s=0}^{p-1} \xi_{s}^{*} \widehat{\psi}_{s}}\right| \Phi\left(t_{p}\right)\right\rangle_{\mathrm{st}},
$$

which means that we take the quantum state $\left|\Phi\left(\xi, t_{p}\right)\right\rangle_{\text {st }}$ over the classical background $\xi$ and collapse all the outgoing modes $\phi_{\text {out }}(m), \ldots, \phi_{\text {out }}(p-1)$ which have emerged by the time moment $t_{p}$, Fig. 30 . As a result, the relevant wavefunction belongs to the space $\mathcal{H}_{\mathrm{s}} \otimes \mathcal{F}_{\text {rel }}$.

Given an environment with a spectral density $J(\omega)$, one computes its memory funNction $M\left(t-t^{\prime}\right)$. Then the incoming/outgoing/relevant modes, and disentanglers $W_{p}$ are found according to the algorithm of sec. IV D. This needs to be done once for a given $J(\omega)$.

Suppose we have a random noise sample $\xi$. The initial condition for evolution is $|\Phi(\xi, 0)\rangle_{\text {rel }}=\left|\phi_{0}\right\rangle_{\mathrm{s}}$, since there are no relevant modes at $t_{0}=0$.

The steps of how $\left|\Phi\left(\xi, t_{p}\right)\right\rangle_{\text {rel }}$ is propagated in time closely mirror those of RG for density matrices of sec. VID, see Fig. 31. The first step, the entangling step, begins by enlarging the Hilbert space $\mathcal{H}_{\mathrm{s}} \otimes \mathcal{F}_{\text {rel }}$ with the states of the incoming mode via the embedding $\left|\Phi\left(\xi, t_{p}\right)\right\rangle_{\mathrm{rel}} \rightarrow\left|\Phi\left(\xi, t_{p}\right)\right\rangle_{\mathrm{rel}+\mathrm{i}}=\left|\Phi\left(\xi, t_{p}\right)\right\rangle_{\mathrm{rel}} \otimes|0\rangle_{\varphi_{\mathrm{in}}(p)}$.
It is followed by the Hamiltonian propagation:

$$
\begin{gathered}
\left|\Phi^{(1)}\left(\xi, t_{p+1}\right)\right\rangle_{\mathrm{rel}+\mathrm{i}}=\left|\Phi\left(\xi, t_{p}\right)\right\rangle_{\mathrm{rel}+\mathrm{i}} \\
-i d t \widehat{H}_{\mathrm{st}}\left(\xi, \tau_{p}\right) \frac{1}{2}\left\{\left|\Phi^{(1)}\left(\xi, t_{p+1}\right)\right\rangle_{\mathrm{rel}+\mathrm{i}}+\left|\Phi\left(\xi, t_{p}\right)\right\rangle_{\mathrm{rel}}\right\},
\end{gathered}
$$

where the Hamiltonian

$$
\begin{aligned}
\widehat{H}_{\mathrm{st}}\left(\xi, \tau_{p}\right) & =\widehat{S}(\xi) \widehat{H}_{\mathrm{st}}\left(\tau_{p}\right) \widehat{S}^{-1}(\xi) \\
= & \widehat{H}_{\mathrm{s}}+\widehat{s}\left\{\widehat{\psi}_{p}^{\dagger}+\xi_{p}^{*}\right\}+\widehat{s}^{\dagger} M(0) \widehat{\psi}_{p} \\
& +\widehat{s}^{\dagger} \sum_{i=1}^{\min (p, m)} M_{i}(p) \widehat{\phi}_{i}
\end{aligned}
$$

takes into account the classical noise of vacuum fluctuations. Here the subscript 'rel $+\mathrm{i}$ ' means that the Hilbert space is enlarged by additional incoming mode $\phi_{\text {in }}(p)$.

The second step, the disentangling step identifies the new outgoing mode $\phi_{\text {out }}(p)$ which has already decoupled:

$$
\left|\Phi^{(2)}\left(\xi, t_{p+1}\right)\right\rangle_{\mathrm{rel}+\mathrm{o}}=\widehat{W}_{p}\left|\Phi^{(1)}\left(\xi, t_{p+1}\right)\right\rangle_{\mathrm{rel}+\mathrm{i}} .
$$

Please note that since we apply the active change of frame $\widehat{W}_{p}$, the quantum content of the outgoing mode $\phi_{\text {out }}(p)$ just replaces the content of $\phi_{\text {in }}(p)$, so that the Hilbert space 'rel $+\mathrm{o}$ ' is actually equal to ' $\mathrm{rel}+\mathrm{i}$ '.

Finally, in the oblivion step the quantum content of the newly formed outgoing mode is discarded (it collapses to the classical noise ):

$$
\left|\Phi\left(\xi, t_{p+1}\right)\right\rangle_{\mathrm{rel}}={ }_{\phi_{\text {out }}\left(t_{p}\right)}\left\langle 0 \mid \Phi^{(2)}\left(\xi, t_{p+1}\right)\right\rangle_{\mathrm{rel}+\mathrm{o}} .
$$




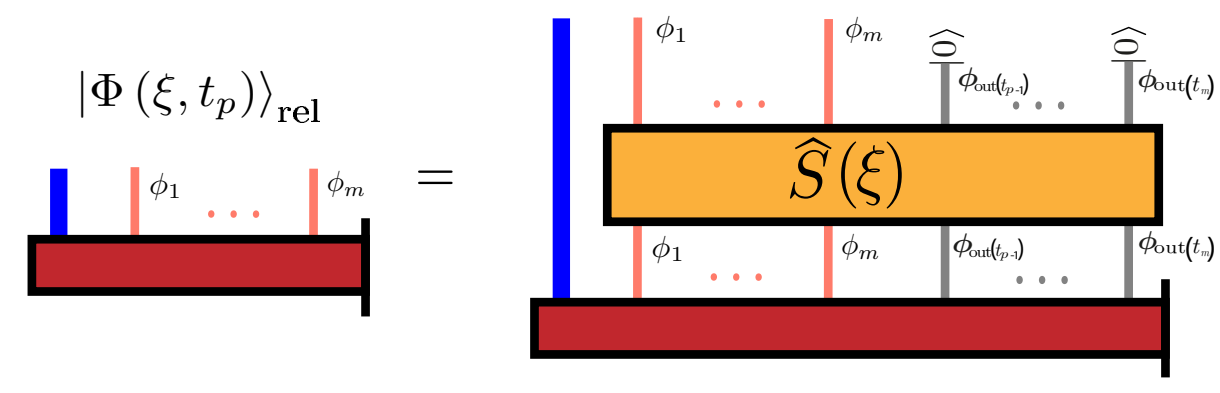

Figure 30. The relevant wavefunction $\left|\Phi\left(\xi, t_{p}\right)\right\rangle_{\text {rel }}$ is defined as a joint pure state of open system and relevant modes. All the outgoing modes $\phi_{\text {out }}(m), \ldots, \phi_{\text {out }}(p-1)$ which have emerged by the time moment $t_{p}$ are projected to vacuum (their quantum content is discarded).

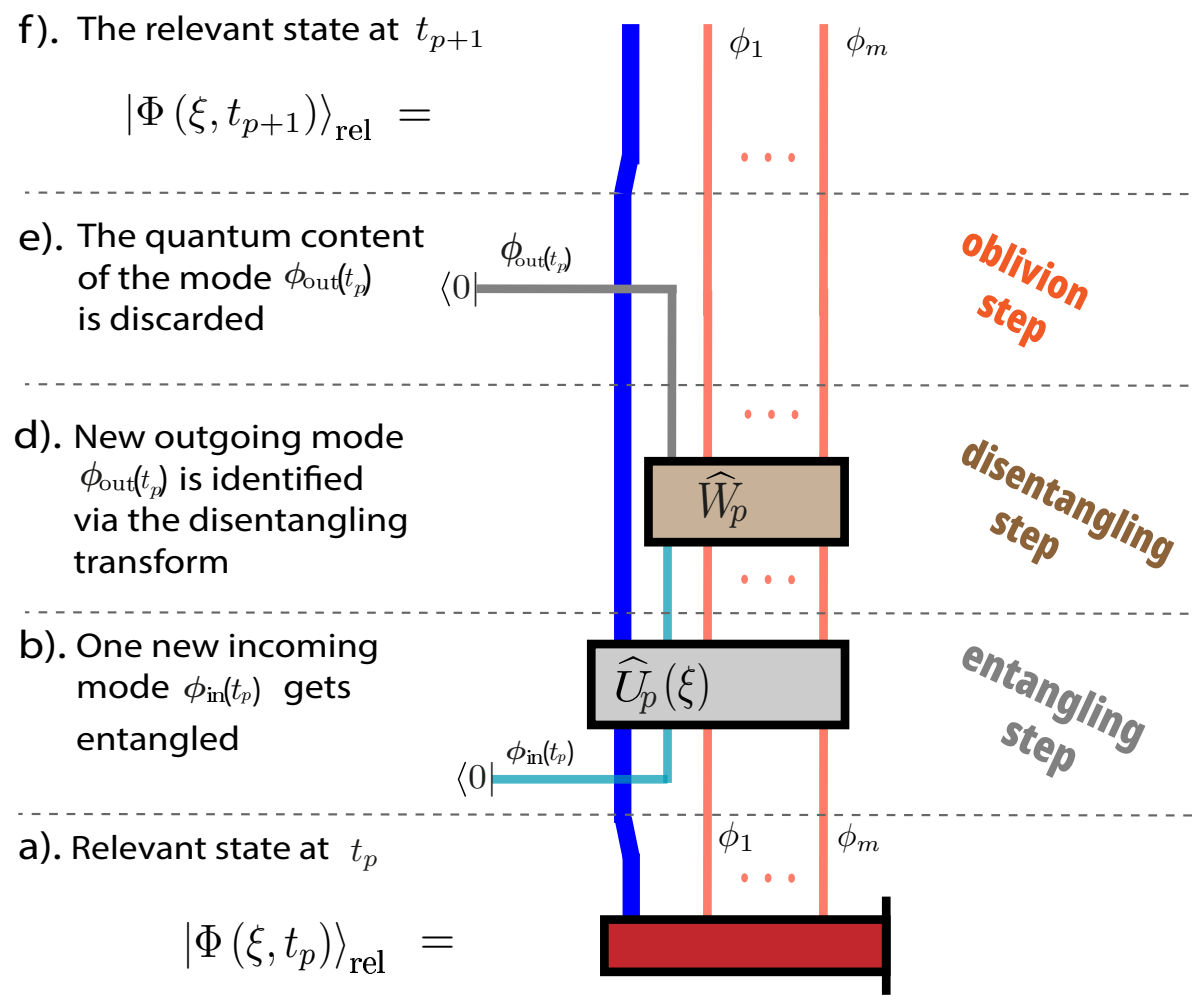

Figure 31. The renormalization group along a quantum trajectory. For each realization (trajectory) of the classical noise $\xi$ of vacuum fluctuations of the environment, the relevant wavefunction $\left|\Phi\left(\xi, t_{p}\right)\right\rangle_{\text {rel }}$ is propagated in time. The full quantum dynamics is obtained as average over $\xi$. The realizations of $\xi$ can be generated via Monte Carlo sampling. On a conceptual level, this structure shows the lifecycle of the quantum complexity during the real-time evolution. First the complexity is generated at the step b). However eventually the quantum complexity is destroyed in the step e). This is because the decoupled entanglement is equivalent to the stochastic ensemble of classical records.

Observe that as a result we return to the Hilbert space $\mathcal{F}_{\text {rel }} \otimes \mathcal{H}_{\mathrm{s}}$. Then the propagation procedure is repeated for the next time moment $t_{p+1}$.

\section{Computing the observables}

The observables $\widehat{o}$ for the open system are computed as

$$
\begin{aligned}
& \left\langle\widehat{o}\left(t_{p}\right)\right\rangle \equiv \operatorname{Tr}\left\{\widehat{o} \widehat{\rho}_{\mathrm{s}}\left(t_{p}\right)\right\}
\end{aligned}
$$

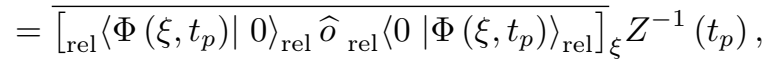


where $|0\rangle_{\text {rel }}$ is the joint vacuum for the relevant modes $\widehat{\phi}_{1}^{\dagger}, \ldots, \widehat{\phi}_{m}^{\dagger}$; the state ${ }_{\text {rel }}\left\langle 0 \mid \Phi\left(\xi, t_{p}\right)\right\rangle_{\text {rel }}$ belongs to $\mathcal{H}_{\mathrm{s}}$; the normalization

$$
Z\left(t_{p}\right)=\overline{\left\|_{\text {rel }}\left\langle 0 \mid \Phi\left(\xi, t_{p}\right)\right\rangle_{\text {rel }}\right\|_{\xi}^{2}}
$$

is equal to 1 in the exact simuation, but should be included in the approximate computation to keep the normalization of the probability. The observables for the environment can also be computed via the mappings (24), $(25)$.

\section{E. Monte-Carlo sampling of vacuum fluctuations}

The realizations of the noise $\xi$ can be sampled stochastically by

$$
\xi_{r}=\sqrt{\Delta \omega} \sum_{k} c\left(\omega_{k}\right) e^{-i \omega_{k} \tau_{r}} z_{k}
$$

where we have introduced a discretization of the frequency axis $\omega_{k}$. The coefficients $c\left(\omega_{k}\right)=\sqrt{J\left(\omega_{k}\right) / \pi}$, which follows from (5) and (8). The complex random numbers $z_{k}$ have the statistics

$$
\overline{z_{k}}=\overline{z_{k}^{*}}=0, \overline{z_{k} z_{l}^{*}}=\delta_{k l} .
$$

We generate a sample of $M$ noise instances $\xi^{1} \ldots \xi^{M}$, and the averages (79), (80) are computed as the sample means.

The states $\left|\Phi\left(\xi, t_{p+1}\right)\right\rangle_{\text {rel }}$ are represented in the Hilbert space $\mathcal{H}_{\mathrm{s}} \otimes \mathcal{F}_{\text {rel }}$ which is truncated in the maximal total occupation of the relevant modes. Such a truncation is expected to converge uniformly on wide time scales if we make the reasonable assumption that there is a balance between the flux of quanta $j_{\text {in }}\left(t_{p}\right)$ being emitted by the open system, and the flux of quanta $j_{\text {out }}\left(t_{p}\right)$ which are eventually discarded in the outgoing modes, see sec. VIF.

\section{F. Importance sampling}

Observe that the Hamiltonian $\widehat{H}_{\text {st }}\left(\xi, \tau_{p}\right)$ is not Hermitean and does not conserve the norm. As a consequence, it may turn out that the ensemble of noise samples (81)-(82) becomes non-representative: the rarest noise realizations recieve the highest weights. This can be fixed by applying an importance sampling technique. Any such technique will require to shift the noise sample, $\xi \rightarrow \xi+\Delta \xi$. In this work we employ the continuous shift of the noise with time. We refer the interested reader to the appendix $\mathrm{D}$ for the derivation. Here we present the resulting simulation procedure.

Given an environment with a spectral density $J(\omega)$, one computes its memory function $M\left(t-t^{\prime}\right)$. Then the incoming/outgoing/relevant modes, and disentanglers $W_{p}$ are found according to the algorithm of sec. IV D. This needs to be done once for a given $J(\omega)$.
The initial condition for the simulation is $|\Phi(\xi, 0)\rangle_{\text {rel }}=$ $\left|\phi_{0}\right\rangle_{\mathrm{s}}$. A noise sample $\xi$ is generated as described in sec. VIIIE. Then the relevant wavefunction $\left|\Phi\left(\xi, t_{p}\right)\right\rangle_{\text {rel }}$ is propagated for the noise sample $\xi$ starting from the initial condition $|\Phi(\xi, 0)\rangle_{\text {rel }}$.

For a given noise sample $\xi$, the open system observable $\widehat{o}$ is averaged at a time $t_{p}$ as

$$
\bar{o}\left(t_{p} ; \xi\right)=\frac{\operatorname{Tr}\left\{\widehat{o} \times_{\mathrm{rel}}\left\langle 0 \mid \Phi\left(\xi, t_{p}\right)\right\rangle_{\mathrm{rel} \mathrm{rel}}\left\langle\Phi\left(\xi, t_{p}\right) \mid 0\right\rangle_{\mathrm{rel}}\right\}}{\left\|{ }_{\mathrm{rel}}\left\langle 0 \mid \Phi\left(\xi, t_{p}\right)\right\rangle_{\mathrm{rel}}\right\|^{2}}
$$

Then the full quantum average of $\widehat{o}$ is given by averaging over $\xi$ :

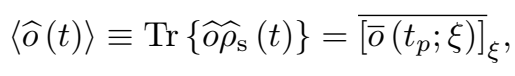

which is implemented by generating a sample of $M$ noise instances $\xi^{1} \ldots \xi^{M}$, and computing the sample mean of $\bar{o}\left(t_{p} ; \xi^{k}\right), k=1 \ldots M$.

The propagation of $\left|\Phi\left(\xi, t_{p}\right)\right\rangle_{\text {rel }}$ is done as follows. Suppose we know $\left|\Phi\left(\xi, t_{p}\right)\right\rangle_{\text {rel }}$ at a time step $t_{p}$. The propagation to the next time moment begins with the entangling step: the embedding $\left|\Phi\left(\xi, t_{p}\right)\right\rangle_{\text {rel }} \rightarrow\left|\Phi\left(\xi, t_{p}\right)\right\rangle_{\text {rel }+\mathrm{i}}=$ $\left|\Phi\left(\xi, t_{p}\right)\right\rangle_{\text {rel }} \otimes|0\rangle_{\varphi_{\text {in }}(p)}$ and the Hamiltonian evolution

$$
\begin{gathered}
\left|\Phi^{(1)}\left(\xi, t_{p+1}\right)\right\rangle_{\mathrm{rel}+\mathrm{i}}=\left|\Phi\left(\xi, t_{p}\right)\right\rangle_{\mathrm{rel}+\mathrm{i}} \\
-i d t \widehat{H}_{\mathrm{st}}^{\prime}\left(\xi, \tau_{p}\right) \frac{1}{2}\left\{\left|\Phi^{(1)}\left(\xi, t_{p+1}\right)\right\rangle_{\mathrm{rel}+\mathrm{i}}+\left|\Phi\left(\xi, t_{p}\right)\right\rangle_{\mathrm{rel}+\mathrm{i}}\right\} .
\end{gathered}
$$

The Hamiltonian $\widehat{H}_{\text {st }}^{\prime}\left(\xi, \tau_{p}\right)$ takes into account the continuous noise shift $f_{p}$,

$$
\begin{aligned}
& \widehat{H}_{\mathrm{st}}^{\prime}\left(\xi, \tau_{p}\right)=\widehat{H}_{\mathrm{s}}+\widehat{s}\left\{\widehat{\psi}_{p}^{\dagger}+\xi_{p}^{*}+f_{p}^{*}\right\} \\
& +\left(\widehat{s}^{\dagger}-\bar{s}^{*}\left(\tau_{p} ; \xi\right)\right)\left\{M(0) \widehat{\psi}_{p}+\sum_{i=1}^{\min (p, m)} M_{i}(p) \widehat{\phi}_{i}\right\} .
\end{aligned}
$$

The shift $f_{p}$ is computed as a convolution of averages $\bar{s}\left(\tau_{p} ; \xi\right)$ of $\widehat{s}$ at all the previous midpoint times $\tau_{p}$ with the memory function:

$$
\begin{aligned}
f_{p}=-i \frac{d t}{2} M\left(\frac{d t}{2}\right) \bar{s}\left(\tau_{p} ; \xi\right) & \\
& -i d t \sum_{l=0}^{p-1} M((p-l) d t) \bar{s}\left(\tau_{l} ; \xi\right) .
\end{aligned}
$$

Here one can approximate $\bar{s}\left(\tau_{l} ; \xi\right) \approx$ $\left\{\bar{s}\left(t_{l+1} ; \xi\right)+\bar{s}\left(t_{l} ; \xi\right)\right\} / 2$, and $\bar{s}\left(t_{l} ; \xi\right)$ is the average of $\widehat{s}$ according to eq. (83). Observe that when solving (85) by iteration, the shift $f_{p}$ should also be recomputed for each iteration since it contains the midpoint term $\bar{s}\left(\tau_{p} ; \xi\right)$ which changes from iteration to iteration. 
The second step, the disentangling step identifies the new outgoing mode $\phi_{\text {out }}(p)$ which has already decoupled:

$$
\left|\Phi^{(2)}\left(\xi, t_{p+1}\right)\right\rangle_{\mathrm{rel}+\mathrm{o}}=\widehat{W}_{p}\left|\Phi^{(1)}\left(\xi, t_{p+1}\right)\right\rangle_{\mathrm{rel}+\mathrm{i}} .
$$

Finally, in the oblivion step the quantum content of the newly formed outgoing mode is discarded (it collapses to the classical noise ):

$$
\left|\Phi\left(\xi, t_{p+1}\right)\right\rangle_{\mathrm{rel}}={ }_{\phi_{\text {out }}\left(t_{p}\right)}\left\langle 0 \mid \Phi^{(2)}\left(\xi, t_{p+1}\right)\right\rangle_{\mathrm{rel}+\mathrm{o}} .
$$

Then the propagation procedure is repeated for the next time moment $t_{p+1}$.

\section{G. Example calculations}

\section{Driven qubit}

Here we provide an example calculation for the model of the driven open quantum system

$$
\widehat{H}_{\mathrm{sb}}(t)=-\frac{\Delta}{2} \widehat{\sigma}_{x}+\widehat{\sigma}_{z} f \cos \omega t+\widehat{\sigma}_{z}\left(\widehat{b}(t)+\widehat{b}^{\dagger}(t)\right),
$$

with the Heaviside-regularized spetral density $J(\omega)=$ $2 \pi \alpha \omega_{\mathrm{c}}^{1-s} \omega^{s} \theta\left(\omega-\omega_{\mathrm{c}}\right)$ for $\alpha=0.1, s=0.5, \Delta=1$, $\omega_{\mathrm{c}}=1, f=0.1, \omega=1$. This leads to the memory function $M(\tau)=\frac{2 \alpha \omega_{\mathrm{c}}^{2}}{s+1} \times \exp \left(-i \tau \omega_{\mathrm{c}}\right) \times{ }_{1} F_{1}\left(1, s+2, i \tau \omega_{\mathrm{c}}\right)$. This situation corresponds to the non-RWA resonant driving and a highly non-Markovian behaviour due to the subohmic spectrum at the origin and because the transition of the open system is at the sharp edge of the band. The is a good benchmark problem since we expect that the resulting dynamics is strongly determined by long-range memory effects. In Fig. 32 we demonstrate that only $m=3$ relevant modes are required to yield almost quantitative agreement. The complete convergence is achieved for $m=4$. In Fig. 33 we demontrate the uniform convergence of $\mathrm{RG}$ with respect to the maximal occupation of the relevant modes, for the same system. Again, 3 quanta yield almost quantitative agreement, and the full convergence is provided by 5 quanta. In order to test our approach, we also solve the Schrodinger equation for this model represented in the semiinfinite chain form [72]. In Fig. 34 we compare the stochastic RG vs the numerical solution of Schrodinger equation (SE) for $\alpha=0.1, s=0.5, \Delta=1, \omega_{\mathrm{c}}=1, f=0.1, \omega=1$. It is seen that the $\mathrm{RG}$ simulation quantitatively reproduces the time-dependence of observable up to steady state.

\section{Information backflow}

When the dynamics of the open quantum system is non-Markovian, it is accompanied by such a phenomenon as information backflow [73-75]. This phenomenon refers to the special time behaviour of the distinguishability of

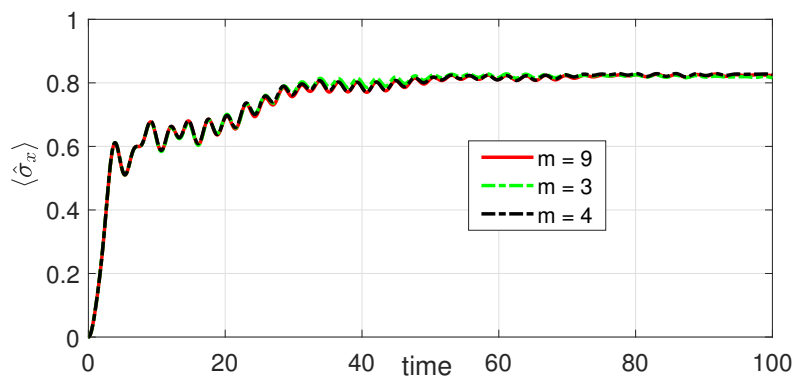

Figure 32. For the system of a driven qubit with a non-RWA coupling to the subohmic environment, good almost quantitative result is provided by $m=3$ relevant modes. The convergence is achieved for $m=4$ relevant modes. The computation is by RG along a quantum trajectory.

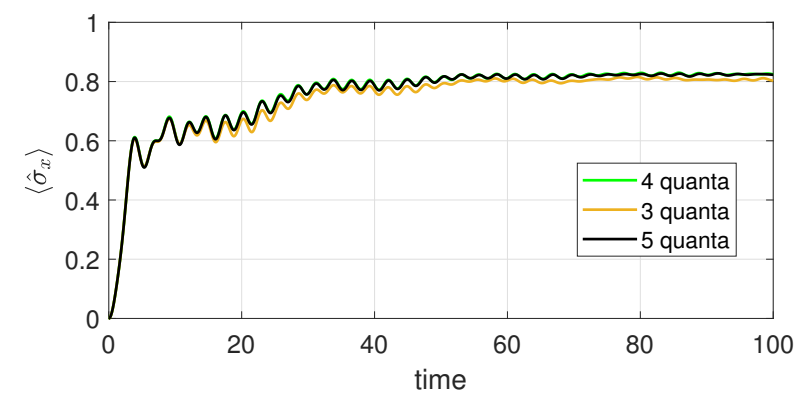

Figure 33. The system of a driven qubit with a non-RWA coupling to the subohmic environment. Uniform convergence of the RG along a quantum trajectory with respect to the maximal number of quanta in the relevant modes.

states of open quantum system. The distiguishability of two quantum states $\widehat{\rho}_{1}(t)$ and $\widehat{\rho}_{2}(t)$ (reduced density matrices) of open quantum system is measured by the trace distance [73]

$$
T\left(\widehat{\rho}_{1}, \widehat{\rho}_{2} ; t\right)=\frac{1}{2} \operatorname{Tr} \sqrt{\left(\widehat{\rho}_{1}(t)-\widehat{\rho}_{2}(t)\right)^{2}} .
$$

In the Markovian regime $T\left(\widehat{\rho}_{1}, \widehat{\rho}_{2} ; t\right)$ should monotonically nonincrease with time for any pair of states [76]. On the contrary, in the non-Markovian mode, we can identify at least one pair of states for which $T\left(\widehat{\rho}_{1}, \widehat{\rho}_{2} ; t\right)$ is increasing for some time interval. This is the information backflow, and is one of the signatures of non-Markovian behaviour.

The concept of relevant modes presented in this paper proposes a physical mechanism behind this phenomemon. As shown in Fig. 26, the relevant modes play the role of a finite imperfect cavity. Then the interaction between the open quantum system and these modes leads to the vacuum Rabi oscillations, i. e. oscillating exchange of energy between the open system and the cavity. These damped oscillations lead to the non-monotonous behaviour of the measures of information backflow. Roughly speaking, when the open quantum system losses its energy, the available phase space shrinks, the states get closer: be- 


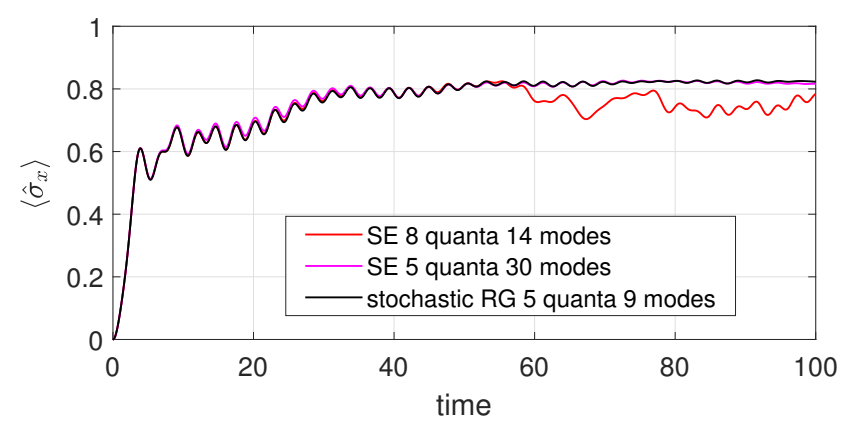

Figure 34. The system of a driven qubit with a non-RWA coupling to the subohmic environment. Comparison of the RG along a quantum trajectory with the solution of Schrodinger equation (SE). In RG the number of relevant modes is $m=9$, the maximal occupation is truncated at $n=5$ quanta. SE was computed for the two set of parameters: to validate the large-time asymptotics, the Fock space was truncated at $n=5$ quanta and $m=30$ sites of the semiinfinite chain; in order to validate the mid-time solution, $n=8$ and $m=14$ was used.

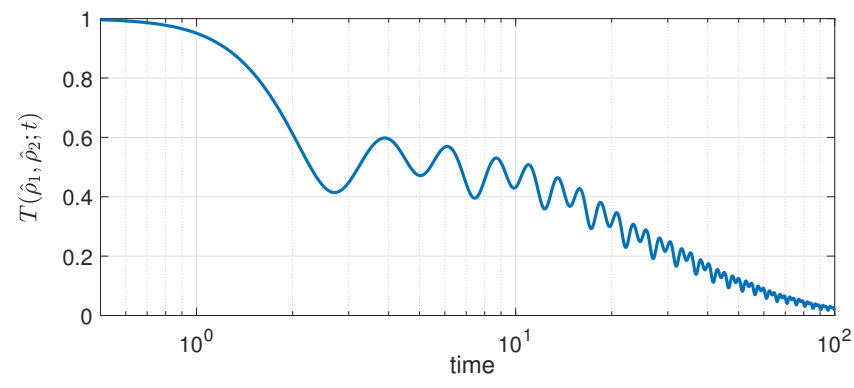

Figure 35. Time dependence for the trace distance $T\left(\widehat{\rho}_{1}, \widehat{\rho}_{2} ; t\right)$ eq. (91) between the initial open systen states $\widehat{\rho}_{1}(0)=|\uparrow\rangle\langle\uparrow|$ and $\widehat{\rho}_{2}(0)=|\downarrow\rangle\langle\downarrow|$ for the model (90) without driving $(f=0)$. The nonmonotonic behaviour inicates that the we are in the non-Markovian regime.

come less distinguishable. However when the energy flow is reversed, the available phase space of open quantum system expands, and the states move away from each other (become more distinguishable).

Here we present the calculation of the time dependence of trace distance (91) for the system (90). We take $\widehat{\rho}_{1}(0)=|\uparrow\rangle\langle\uparrow|$ and $\widehat{\rho}_{2}(0)=|\downarrow\rangle\langle\downarrow|$. In Fig. 35 we present the plot of $T\left(\widehat{\rho}_{1}, \widehat{\rho}_{2} ; t\right)$ for the model (90) without driving $(f=0)$ : we observe the periodic increase of distinguishability, so we are indeed in the non-Markovian regime.

\section{Comparison with the tensor network methods}

Here we provide a numerical comparison with the timeevolving matrix product operator (TEMPO) method [28]. TEMPO is a state-of-the-art approach for the computation of open quantum system dynamics. It is used in

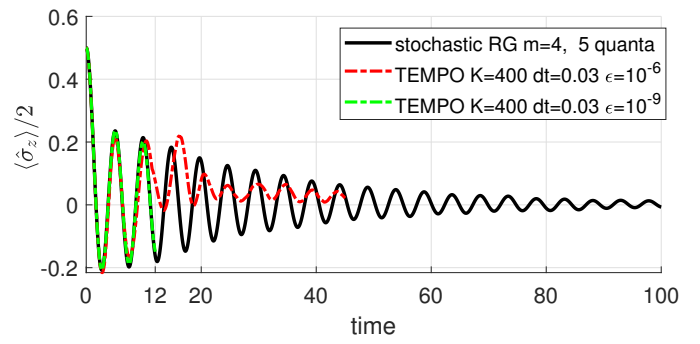

Figure 36. Comparison of stochastic RG along trajectories and TEMPO. Time dependence of $\left\langle\widehat{\sigma}_{z}\right\rangle / 2$ is computed for model (90) without driving $(f=0)$. Initial state is $\widehat{\rho}_{\mathrm{s}}(0)=$ $|\uparrow\rangle\langle\uparrow|$. Stochastic RG (black line) is for $m=4$ relevant modes and is truncated at $n=5$ quanta. It yields the converged result on the presented time scales. The TEMPO (dashed green and red lines) employs a matrix product operator of length $K=400$ with bond dimensions up to 358 . However it can capture correctly the dynamics only up to the revival time $t_{\text {cut }}=K d t=12$, with $d t=0.03$. TEMPO with SVD treshold $\epsilon=10^{-9}$ (green dashed line) agrees with the stochastic RG results before the revival at $t_{\text {cut }}$. However each time step $d t=0.03$ becomes rather time consuming $(\approx 40 \mathrm{~min}$ on a 40 CPU core cluster). TEMPO with larger SVD treshold $\epsilon=10^{-6}$ (red dashed line) starts to deviate even before $t_{\text {cut }}$, but can be efficiently propagated to larger times, so that we see clearly see the revival behaviour.

recent works e.g. to calculate the quantum heat statistics [77]. We use the package provided by the authors [78]. TEMPO represents the correlated state of the open system and the environment as a matrix product operator (MPO) state [28]. It depends on parameters $d t, K$, and $\epsilon$. The evolution is discretized with a time step $d t$. The length $K$ of the MPO corresponds to cutoff time scale $t_{\text {cut }}=K d t$. All the correlation effects of the environment at time scales larger than $t_{\text {cut }}$ are neglected. Finally, $\epsilon$ defines the treshold below which the SVD components are discarded. In Fig. 36 we present the time-dependence of $\left\langle\widehat{\sigma}_{z}\right\rangle / 2$ computed by the RG along a quantum trajectory and compare it with the TEMPO computation. The system under consideration is again given by eq. (90) without driving $(f=0)$. We start from $\widehat{\rho}_{\mathrm{s}}(0)=|\uparrow\rangle\langle\uparrow|$. We observe that only $m=4$ relevant modes at a finite occupation (5 quanta) is enough to achieve the converged time dependence on large time scales. At the same time, the finite length $K$ of the TEMPO corresponds to the finite revival time $t_{\text {cut }}$ after which the computed evolution is corrupted. The longer we want to simulate the dynamics, the longer MPO should we employ. TEMPO cannot correctly capture the large-time asyptotics with a finite number of variables. Also, TEMPO requires rather small SVD treshold $\epsilon=10^{-9}$ in order to quantitatively reproduce the observable properties of the open quantum system. This leads to a rapid growth of the bond dimension, Fig. 37 The computational time per one time step $(d t=0.03)$ also increases. As a result of these two effects (increasing length of MPO with the increasing bond dimensions), the large time asymptotics of nonstation- 


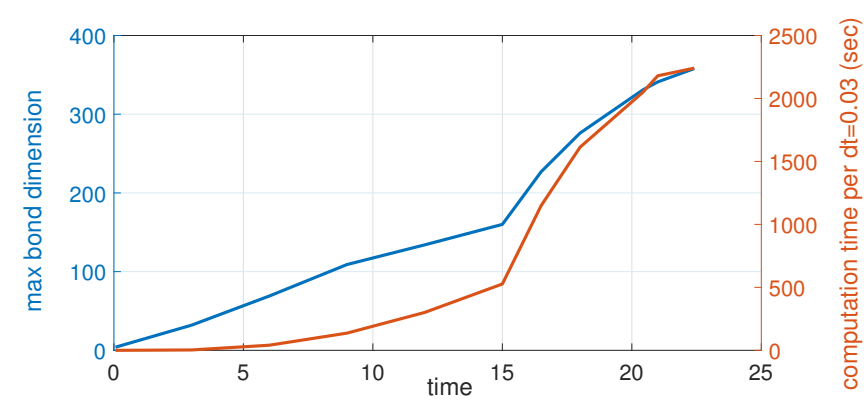

Figure 37. For the same situation as in previous Fig. 36. The maximal bond dimension of TEMPO $(K=400, \epsilon=$ $\left.10^{-9}, d t=0.03\right)$ is growing with the propagation time. This growth is accompanied with the increase in the computation time per one time step $d t$.

ary evolution is a computationally hard property for the state-of-the-art tensor network methods. That is why the conjectured boundedness of complexity (as a function of simulation time) for the proposed RG methods is a nontrivial result.

\section{ENTANGLEMENT IN THE TIME DOMAIN}

In the previous sections we repeatedly refer to the entanglement as a temporal property. For example, we say that the outgoing mode $\phi_{\text {out }}(t)$ is not entangled to the future (after $t$ ) in Fig. 1 We also introduce the entanglers and disentanglers in Figs. 15 and 17. However the cautious reader may notice that the entanglement is usually considered as a spatial property and not a temporal one. The reason is that the entanglement is defined by dividing a quantum system into parts with strictly independent degrees of freedom. Due to the non-locality of the time degrees of freedom, Fig. 14, it is impossible to perform such a strict partitioning in the time domain. Thus, the question arises, what is the exact content of our concept of temporal entanglement.

\section{A. Absence of entanglement}

We believe there are no doubts how should one define the absence of entanglement. Suppose we have a pure state $\left|\Phi\left(t_{p}\right)\right\rangle_{\text {st }}$ which depends on the time cell degrees of freedom $\widehat{\psi}_{0}^{\dagger}, \ldots, \widehat{\psi}_{p-1}^{\dagger}$ and on the quantum numbers of the open system. Let us divide it into the two subsystems. The subsystem $A$ consists of the open quantum system and of the $q$ most recent time cells $\widehat{\psi}_{p-q}^{\dagger}, \ldots, \widehat{\psi}_{p-1}^{\dagger}$. The subsystem $B$ is chosen to contain the remaining time cells $\widehat{\psi}_{0}^{\dagger}, \ldots, \widehat{\psi}_{p-q-1}^{\dagger}$. There is no entanglement between $A$ and $B$ if after tracing out the $B$ 's degrees of freedom the reduced state of $A$ is still pure. Recalling the trace relation (31) for the tape model, we have for the reduced density matrix of $A$ :

$$
\widehat{\rho}_{A}=\tau_{\tau_{p-q-1}}\left\langle 0\left|\cdots_{\tau_{0}}\left\langle 0\left|\widehat{\rho}_{A \mid B}\right| 0\right\rangle_{\tau_{0}} \ldots\right| 0\right\rangle_{\tau_{p-q-1}},
$$

where $|0\rangle_{\tau_{k}}$ is the vacuum of mode $\widehat{\psi}_{k}^{\dagger}$, and

$$
\begin{array}{r}
\widehat{\rho}_{A \mid B}=\left\{e^{\sum_{r=0}^{p-q-1} \sum_{s=p-q}^{p-1}\left(\widehat{\psi}_{r}^{\dagger} M_{r s} \widehat{\psi}_{s}+\text { c.c. }\right)} e^{\sum_{r s=0}^{p-q-1} \widehat{\psi}_{r}^{\dagger} M_{r s} \widehat{\psi}_{s}}\right. \\
\left.\times:\left|\Phi\left(t_{p}\right)\right\rangle_{\text {st st }}\left\langle\Phi\left(t_{p}\right)\right|:\right\}_{A}
\end{array}
$$

takes into account the pairings between the time cells of $B$ and the cross-pairings between the time-cells of $A$ and $B$. Observe that there is no pairings between the time cells of $A$ in $\widehat{\rho}_{A \mid B}$ : otherwise $\widehat{\rho}_{A}$ could be mixed irrespectively of its entanglement to $B$. We say that there is no entanglement between $A$ and $B$ if $\widehat{\rho}_{A}$ is pure, $\widehat{\rho}_{A}=$ const $\times|f\rangle_{A A}\langle f|$. In other words, after tracing out $B$ we still can consider that $A$ is defined by a pure state $|f\rangle_{A}$.

One example situation when $A$ and $B$ are not entangled is the state of the form

$$
\left|\Phi\left(t_{p}\right)\right\rangle_{\text {st }}=|f\rangle_{A} \otimes\left|f_{\text {out }}\left(t_{p-q-1}\right)\right\rangle_{B},
$$

where in the state $\left|f_{\text {out }}\left(t_{p-q-1}\right)\right\rangle_{B}$ only the outgoing modes $\phi_{\text {out }}(m), \ldots, \phi_{\text {out }}(p-q-1)$ are populated by quanta. Then the cross-pairings in eq. (93) are negligible by construction of the incoming and outgoing streams in sec. IV D, and $\widehat{\rho}_{A} \propto|f\rangle_{A{ }_{A}}\langle f|$ is pure. This is the exact meaning of saying that the outgoing modes at time $t_{p-q-1}$ are not entangled to the future incoming modes.

\section{B. Measure of temporal entanglement}

There are situations when we need to measure the amount of entanglement between the subsystems. For example, one usually wants to know whether the entanglement is short-range or long-range. If the entanglement is long-range, then it is interesting to know its asymptotical scaling. Therefore, here we propose a measure of temporal entanglement.

The entanglement is measured by the entropy of distribution of non-zero eigenvalues over the ensemble of eigenvectors. If $\widehat{\rho}_{A}$ (from eq. (92)) is pure then this distibution is localized on a single eigenvector: the entropy is zero and there is no entanglement. In the opposite case of a maximally mixed $\widehat{\rho}_{A}$, the distribution is evenly smeared over the eigenvectors: the entropy is maximal and so is the entanglement.

Therefore, we define the entanglement entropy as

$$
\begin{aligned}
S_{A \mid B}=-\log \operatorname{Tr}_{A} & \left\{\left|\widehat{\rho}_{A}\right| / \operatorname{Tr}_{A}\left|\widehat{\rho}_{A}\right|\right\}^{2} \\
= & 2 \log \operatorname{Tr}_{A}\left|\widehat{\rho}_{A}\right|-\log \operatorname{Tr}_{A}\left|\widehat{\rho}_{A}\right|^{2}
\end{aligned}
$$

Here we base our definition on the Renyi entropy of the second order. The absolute value $\left|\widehat{\rho}_{A}\right|$ is employed because $\widehat{\rho}_{A}$ may have negative eigenvalues. This is due to the restriction of the set of pairings, see sec. VIB. Also 


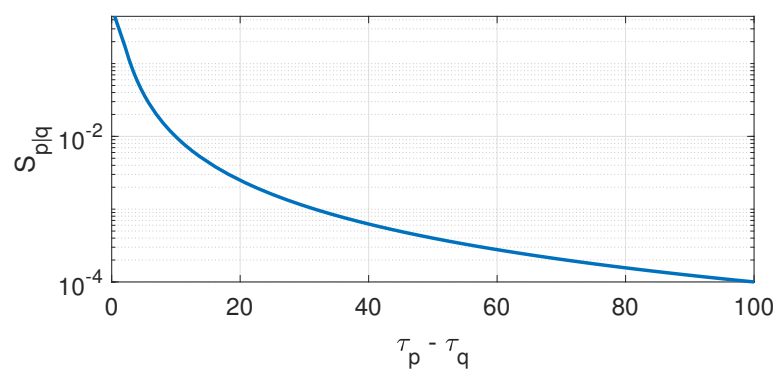

Figure 38. The entanglement entropy $S_{p \mid q}$ of the state $\widehat{\psi}_{p}^{\dagger} \widehat{\psi}_{q}^{\dagger}|0\rangle_{\mathrm{t}}$ with two quanta emitted at $\tau_{p}$ and $\tau_{p}, \tau_{p}>\tau_{q}$. It is a long-range function of the time distance $\tau_{p}-\tau_{q}$ : $S_{p \mid q} \propto\left|M_{p q}\right|^{2} \propto\left(\tau_{p}-\tau_{q}\right)^{-s}$. The plot is for the case of Ohmic bath with the memory function $\frac{1}{2}(1+i t)^{-2}$. Observe that althrough the state $\widehat{\psi}_{p}^{\dagger} \widehat{\psi}_{q}^{\dagger}|0\rangle_{\mathrm{t}}$ seems to be factorized, actually the states of emitted quanta are non-local in time, see. Fig. 14. That is why when we trace out one of the time cells, the reduced state of the other time cell becomes mixed: the quanta are distributed non-locally between the both time cells.

we normalize the density matrix by $\operatorname{Tr}\left|\widehat{\rho}_{A}\right|$ because $\widehat{\rho}_{A}$ is in general not normalized, $\operatorname{Tr}_{A} \widehat{\rho}_{A} \neq 1$. Here, $\operatorname{Tr}_{A}$ is the trace in the conventional sense, over some basis in the Fock space of the time cells $\mathcal{F}_{\mathrm{t}}$.

Our definition of the entanglement entropy reduces to the conventional Renyi entropy in the limit of infiniteband environment with $M\left(t-t^{\prime}\right)=\delta\left(t-t^{\prime}\right)$. It is reasonable because in this limit the time cells are local independent degrees of freedom, and the conventional partitioning into the independent subsystems is possible.

\section{Entanglement is long-range in time}

Let us consider as a simple example the pure state created by the two emission events: one at $\tau_{p}$ and the other at $\tau_{q}$, with $\tau_{p}>\tau_{q}$,

$$
|\Phi\rangle_{\mathrm{t}}=\widehat{\psi}_{p}^{\dagger} \widehat{\psi}_{q}^{\dagger}|0\rangle_{\mathrm{t}}
$$

Let us compute the entanglement entropy $S_{p \mid q}$ between these two time moments. Applying (92), (93), and (95), we find:

$$
S_{p \mid q}=2 \log \left(p_{1}+p_{2}\right)-\log \left(p_{1}^{2}+p_{2}^{2}\right),
$$

where $p_{1}=M(0) /\left(M(0)+\left|M_{p q}\right|^{2}\right), \quad p_{2}=$ $\left|M_{p q}\right|^{2} /\left(M(0)+\left|M_{p q}\right|^{2}\right)$. At a large time interval between the emission times the memory function decays as inverse power law, $\left|M_{p q}\right|^{2} \propto\left(\tau_{p}-\tau_{q}\right)^{-s}$, and the entanglement entropy aslo decays according to the inverse power law $S_{p \mid q} \propto\left|M_{p q}\right|^{2} \propto\left(\tau_{p}-\tau_{q}\right)^{-s}$. In Fig. 38 we present the plot of $S_{p \mid q}$ vs $\tau_{p}-\tau_{q}$ for the Ohmic memory function (10) with $\alpha=1, \omega_{\mathrm{c}}=1, s=1$.

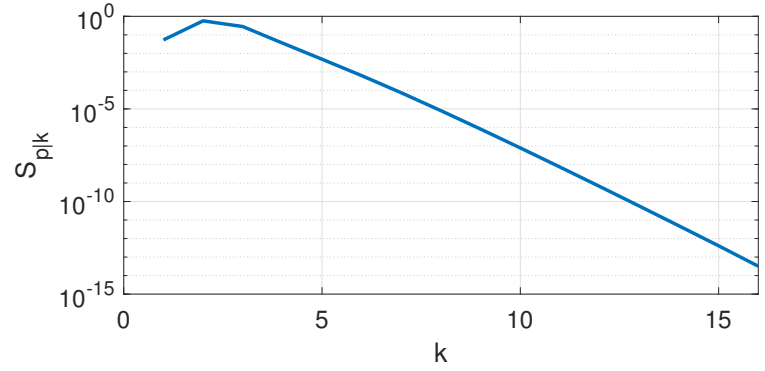

Figure 39. The entanglement entropy $S_{p \mid k}$ of the state $\widehat{\psi}_{p}^{\dagger} \widehat{\phi}_{k}^{\dagger}|0\rangle_{\mathrm{t}}$ with one quantum emitted at $\tau_{p}$, and the other quantum in the fastest decoupling basis state $\phi_{k}$, computed for the time moment $t_{p}$, see the section IVB. . The case of Ohmic bath with the memory function $\frac{1}{2}(1+i t)^{-2}$. The plot is for $S_{p \mid k}$ wrt the fastest decoupling basis element number $k$. It is seen that the entanglement entropy decays exponentially fast with $k$. Therefore, in the frame of incoming/relevant/outgoing modes the entanglement becomes effectively finite-ranged.

\section{Entanglement is finite-range in the frame of incoming/relevant/outgoing modes}

Now let us consider the pure state $|\Phi\rangle_{\mathrm{t}}=\widehat{\psi}_{p}^{\dagger} \widehat{\phi}_{k}^{\dagger}|0\rangle_{\mathrm{t}}$ when one quantum is emitted at a time moment $\tau_{p}$, and the other is in the fastest decoupling basis state $\phi_{k}$, computed for the time moment $t_{p}$, see the section IV B. The formula (97) for the entanglement entropy still holds, with the substitution $S_{p \mid q} \rightarrow S_{p \mid k}$ and $M_{p q} \rightarrow\left(p|M| \phi_{k}\right)$. In Fig. 39 we plot the dependence of $S_{p \mid k}$ with respect to the fastest decoupling basis element number $k$. We see that in the frame of incoming/relevant/outgoing modes the entanglement becomes effectively finite-ranged.

\section{CONCLUSION}

Structure of entanglement. In this paper we propose a constructive way of how to characterize the structure of entanglement. By tracking the degrees of freedom (the "bricks") which couple (for the first time) to the evolution and decouple (irreversibly) from the evolution, we obtain a useful and intrinsic (free from adhoc assumptions) characterization. Then the entanglement starts to look like a Lego built from these bricks. Here the evolution can be understood in a generalized sense, e.g. as an RG flow parameter.

Real-time motion of open quantum systems. In this paper we describe the entire lifecycle of the entanglement: from its generation through the entanglement of incoming modes to its death in the oblivion of outgoing modes. This sheds light on a number of questions. First, this shows that the "true" quantum complexity $[3,4,46,79,80]$ of the real-time motion survives only in the modes of the environment which are non-negligibly coupled to the future quantum motion. If there is a bal- 
ance between the flux of emitted quanta and the flux of quanta which are forgotten in the outgoing modes, then this complexity is asymptotically bounded on large times. Then the real-time motion of open quantum system becomes efficiently computable on a classical computer. Second, we find how the decoherence happens continuously in the non-Markovian regime. We find how tiny parts of quantum information decouple and disappear each infinitesimal time moment. Interesting future direction is to study the distributed open quantum systems when there is a number $r$ of coupling sites $\widehat{b}_{\alpha}^{\dagger}(t)$, $\alpha=1 \ldots r$, to the environment. We expect that the presented approach should be straightforwardly exended to this case. The main modification is that the memory function (8), the matrix $K(p)$ eq. (18), and the relevant modes will acquire additional indices $\alpha, \alpha^{\prime}=1 \ldots r$. The imporant question is whether it is possible to construct low-dimensional relevant modes inside the distributed open system so as to escape the growth of its Hilbert space dimension.

Few-mode approximations of non-Markovian environments. It turns out that each moment of time only a finite number $m$ of degrees of freedom of the environment are significantly coupled to the future motion. Moreover, the significance of the neglected degrees of freedom decreases exponentially fast. This opens interesting perspective on the few-mode approximations of the environments with memory.

Renormalization group methods. We also obtain a novel perpective for the renormalization group methods. Traditionally these methods are based on the iterative application of the scale and coarsegraining tranformations. Here we go beyond this paradigm. Any evolution can be considered as a certain RG flow provided it is accompanied by the continuous emergence of degrees of freedom which are not entangled to the future evolution. These degrees of freedom are irrelevant and can be iteratively traced out. Then the relevant subspace of such an $\mathrm{RG}$ procedure is not the conventional infrared limit, but the subspace of degrees of freedom which are significantly coupled to the future evolution. We illustrate this point (down to the resulting numerical schemes) for the case of real-time flow for open system. To extend this ideas to other many-body problems is a subject of future research.

Emergence of classical records. There is a interesting interrelationship between the entanglement, $\mathrm{RG}$, and the models of continuous measurement [81]. The irrelevant degrees of freedom (which are not entangled to the future), can be collapsed [82, 83] to a classical measurement signal as soon as they emerge. Computationally, this leads to a stochastic RG along a quantum trajectory. The numerical benefit over the deterministic RG for density matrices is that the latter scales as $N^{2}$ for $N$ being the dimension of the relevant Fock subspace, whereas the former scales as $N$.

Quantum trajectories. In this work we promote a constructive viewpoint on quantum trajectories [84-89]: we propose to define the quantum trajectory as a model of how the quantum complexity decays into the classical complexity of a stochastic ensemble.

These results are illustrated with a number of calculations for the model of a two-level system (qubit), with and without driving, which is coulped to the environments with subohmic/Ohmic/superohmic and semicircle spectral densities. In particular, in the cases considered only 4-3 relevant modes were enough to achieve the numerical convergence.

Of couse, we illustrate these ideas on a single specific model: the spin in the bosonic bath. However we believe the picture presented in the Introduction, and the concept of irreversible decoupling as negligible average intensity of future interactions (sec. IV), are rather physically transparent and general. They can be applied to a varienty of other models and situations, although specific formal implementation may differ.

\section{ACKNOWLEDGMENTS}

The work was carried out in the framework of the Roadmap for Quantum computing in Russia

\section{Appendix A: SOLVING EQUATIONS IN THE TRUNCATED FOCK SPACE}

The total Hilbert space for the procedure in sec. VID has the basis elements

$$
|q\rangle_{\mathrm{s}} \otimes\left|n_{1}\right\rangle_{1} \otimes\left|n_{2}\right\rangle_{2} \otimes \ldots\left|n_{m+1}\right\rangle_{m+1},
$$

where $|q\rangle_{\mathrm{s}}$ is some basis in the Hilbert space $\mathcal{H}_{\mathrm{s}}$ of the open system; $\left|n_{i}\right\rangle_{i}$ is the state of $n_{i}$ quanta in mode $i$. The modes $i=1 \ldots m$ are our relevant modes. The mode $m+1$ becomes coupled only after $t=t_{m}$ and alternatively plays the role of incoming and outgoing mode.

We truncate the Hilbert space at certain number $n$ of quanta: only the basis states with $\sum_{i} n_{i} \leq n$ are being kept. Let us assume that $N$ is the total number of remaining basis elements. All the basis elements (A1) are numbered:

$$
|k\rangle=\left|q^{(k)}\right\rangle_{\mathrm{s}} \otimes\left|n_{1}^{(k)}\right\rangle_{1} \otimes\left|n_{2}^{(k)}\right\rangle_{2} \otimes \ldots\left|n_{m+1}^{(k)}\right\rangle_{m+1}
$$

for $k=1 \ldots N$. Then the joint many-particle wavefunction $|\Psi\rangle$ of open system and the modes is represented as $|\Psi\rangle=\sum_{k=1}^{N} \Psi_{k}|k\rangle$ with $\Psi_{k}$ being the $1 \times N$ array of complex numbers. The density matrix is represented as $\widehat{\rho}=\sum_{k l=1}^{N} \rho_{k l}|k\rangle\langle l|$, with $\rho_{k l}$ being the $N \times N$ Hermitean matrix of complex numbers.

The operators of open system, the creation/annihilation operators for modes are represented as sparce matrices in the basis of $|k\rangle$. Therefore, all the operations (49)-(52) are implemented in terms of space matrix multiplications. 
Please observe that at the time $t_{p}$ the mode $\min (m+1, p)$ play the role of incoming mode before the disentangling step (51). After the disentangling step the mode min $(m+1, p)$ plays the role of outgoing mode. The oblivion step (52) is implemented by setting to zero all elements of $\rho_{k l}$ which correspond to non-zero occupation of the mode $\min (m+1, p)$. After the oblivion step, at the next time moment, $p \rightarrow p+1$, the mode $\min (m+1, p)$ becomes again the incoming mode.

\section{Appendix B: COMPUTING THE PAIRING UPDATE}

Our propagation procedure is permitted to have a local error $O\left(d t^{3}\right)$. Then it is sufficient to compute the pairing update (50) up to the error $O\left(d t^{3}\right)$. Observe how the pairing update (50) is acting: if we consider the Taylor series of the pairing function, then at the order $n$ it annihilates at least $n$ quanta in the incoming mode, either from the left (via $\widehat{\psi}_{p}$ ) or from the right (via $\widehat{\psi}_{p}^{\dagger}$ ) of the density matrix $\widehat{\rho}_{\text {rel }+\mathrm{i}}^{(1)}\left(t_{p+1}\right)$. However, the amplitude to find $n$ quanta in the incoming mode scales as $d t^{n}$. Indeed, looking at the propagation (49) and the Hamiltonian $\widehat{H}_{\mathrm{st}}\left(\tau_{p}\right)$, eq. $(34)$, we see that the new quanta are created via $\propto d t \widehat{\psi}_{p}^{\dagger}$. Therefore, with the second order accuracy we compute the pairing update (50) as

$$
\widehat{\rho}_{\mathrm{rel}+\mathrm{i}}^{(2)}\left(t_{p+1}\right)=\widehat{\rho}_{0}+\widehat{\rho}_{1}+\frac{1}{2} \widehat{\rho}_{2}
$$

where

$$
\widehat{\rho}_{0}=\widehat{\rho}_{\mathrm{rel}+\mathrm{i}}^{(1)}\left(t_{p+1}\right)
$$

and the recurrent relation between $\widehat{\rho}_{i}$ and $\widehat{\rho}_{i-1}$,

$$
\begin{aligned}
\widehat{\rho}_{i}=M(0) \widehat{\psi}_{p} \widehat{\rho}_{i-1} \widehat{\psi}_{p}^{\dagger} & \\
& +\sum_{i} M_{i}(p) \widehat{\phi}_{i} \widehat{\rho}_{i-1} \widehat{\psi}_{p}^{\dagger} \\
& +\sum_{i} M_{i}^{*}(p) \widehat{\psi}_{p} \widehat{\rho}_{i-1} \widehat{\phi}_{i}^{\dagger},
\end{aligned}
$$

for $i=1,2$.

\section{Appendix C: COMPUTING THE DISENTANGLER TRANSFOM}

Here we present the algorithm we use in order to apply the disentangler transforms (51) and (78). The latter are defined as a unitary Bogoliubov transform, eq. (36). The Bogoliubov transform has the general form

$$
\widehat{W}=\exp \left(i \sum_{k l} \widehat{\psi}_{k}^{\dagger} h_{k l} \widehat{\psi}_{l}\right) \equiv \exp \left(i \widehat{H}_{W}\right)
$$

with suitably defined $\widehat{\psi}_{l}, \widehat{\psi}_{l}^{\dagger}$ and a hermitean matrix $h_{k l}$. Here we introduce the effective "Hamiltonian" $\widehat{H}_{W}$ which generates $\widehat{W}$. We need to apply $\widehat{W}$ to some wavefunction $|\Psi\rangle$

$$
\left|\Psi_{W}\right\rangle=\widehat{W}|\Psi\rangle
$$

In general, $h_{k l}$ is a dense matrix (as it turns out to be in eq. (36)). Therefore, each application of $\widehat{H}_{W}$ is expensive if $|\Psi\rangle$ is many-particle. We need to devise an algorithm which minimizes the number of applications of $\widehat{H}_{W}$.

Suppose that our budget is $M$ applications of $\widehat{H}_{W}$. Then we construct the orthonormal basis $\left|\Phi_{0}\right\rangle \ldots\left|\Phi_{M}\right\rangle$ from $M+1$ vectors $|\Psi\rangle, \widehat{H}_{W}|\Psi\rangle, \ldots, \widehat{H}_{W}^{M}|\Psi\rangle$. The basis is constructed recurrently. We begin as

$$
\left|\Phi_{0}\right\rangle=|\Psi\rangle /\|\Psi\|
$$

where we normalize $|\Psi\rangle$ since it is normalizated differently in the tape model, see eq. (45). Now suppose we know $\left|\Phi_{k}\right\rangle$. We apply $\widehat{H}_{W}$ and find new unnormalized basis element $\left|\widetilde{\Phi}_{k+1}\right\rangle$ :

$$
\begin{array}{r}
\left|\Phi_{k}^{H}\right\rangle=\widehat{H}_{W}\left|\Phi_{k}\right\rangle, \beta_{k}=\left\langle\Phi_{k} \mid \Phi_{k}^{H}\right\rangle, \\
\left|\widetilde{\Phi}_{k+1}\right\rangle=\left|\Phi_{k}^{H}\right\rangle-\beta_{k}\left|\Phi_{k}\right\rangle-\alpha_{k-1}\left|\Phi_{k-1}\right\rangle \\
\alpha_{k}=\left\|\widetilde{\Phi}_{k}\right\|,
\end{array}
$$

were we set $\alpha_{-1}=0,\left|\Phi_{-1}\right\rangle=0$. Starting from $k=1$, we additionally orthogonalize $\left|\widetilde{\Phi}_{k+1}\right\rangle$ to all the previous basis elements:

$$
\left|\Phi_{k+1}^{\perp}\right\rangle=\left(1-\left|\Phi_{k}\right\rangle\left\langle\Phi_{k}\right|\right) \ldots\left(1-\left|\Phi_{0}\right\rangle\left\langle\Phi_{0}\right|\right)\left|\widetilde{\Phi}_{k+1}\right\rangle .
$$

Otherwise the procedure will be numerically unstable. Finally, we normalize the basis element:

$$
\left|\Phi_{k+1}\right\rangle=\left|\Phi_{k+1}^{\perp}\right\rangle / \|\left|\Phi_{k+1}^{\perp}\right\rangle \| .
$$

As a result, $\widehat{H}_{W}$ is represented as a tridiagonal matrix

$$
H_{\mathrm{ch}}=\left[\begin{array}{cccc}
\beta_{0} & \alpha_{0} & 0 & 0 \\
\alpha_{0} & \beta_{1} & \alpha_{1} & 0 \\
0 & \alpha_{1} & \ddots & \vdots \\
\vdots & \ddots & \ddots & \alpha_{M-1} \\
0 & 0 & \alpha_{M-1} & \beta_{M}
\end{array}\right]
$$

We see from this form that the Hamiltonian $\widehat{H}_{W}$ is represented as a semiinfinite chain attached to $\left|\Phi_{0}\right\rangle$. The hopping between the sites of the chain is given by $\alpha_{k}$. Therefore, we obtain the first criterion for truncation: if $\alpha_{k}^{2}$ is below some treshold, e.g. $10^{-5}$, then the remaining sites are effectively decoupled, and we can stop at $k$ applications of $\widehat{H}_{W}$ and set $M=k$. The second criterion 
is the norm $\|\left|\Phi^{\perp}{ }_{k+1}\right\rangle \|^{2}$ : if it is below some treshold e.g. $10^{-4}$, then the new basis elements are essentially linerarly dependent, so we stop at $k$ applications of $\widehat{H}_{W}$ and set $M=k$.

Finally, the $M \times M$ tridiagonal matrix $H_{\mathrm{ch}}$, eq. $(\mathrm{C} 7)$, is diagonalized:

$$
H_{\mathrm{ch}}=U\left[\begin{array}{ccc}
\omega_{1} & \ldots & 0 \\
\vdots & \ddots & \vdots \\
0 & \ldots & \omega_{M}
\end{array}\right] U^{\dagger}
$$

and the approximation $\left|\Psi_{W}^{M}\right\rangle$ to $\left|\Psi_{W}\right\rangle$ is computed as

$$
\left|\Psi_{W}^{M}\right\rangle=\sum_{k, l, l^{\prime}=1}^{M} \exp \left(-i \omega_{k}\right) U_{l k}\left|\Phi_{l}\right\rangle U_{l^{\prime} k}^{*}\left\langle\Phi_{l^{\prime}} \mid \Psi\right\rangle .
$$

If $\alpha_{k}^{2}$ and $\|\left|\Phi^{\perp}{ }_{k+1}\right\rangle \|^{2}$ are still above their tresholds, then the last, fourth criterion, is that we stop adding basis elements when $\|\left|\Psi_{W}^{M}\right\rangle-\left.\left|\Psi_{W}^{M+1}\right\rangle\right|^{2}$ is below some treshold e.g. $10^{-3}$. Then we set $\left|\Psi_{W}\right\rangle \approx\left|\Psi_{W}^{M}\right\rangle$. For the numerical computations in the paper we obtain that $M$ lies in the range $3 \ldots 16$.

\section{Appendix D: IMPORTANCE SAMPLING}

The noise shift leads to an additional Bogoliubov transform in the state vector (74):

$$
S(\Delta \xi)=e^{\sum_{s=0}^{\infty} \Delta \xi_{s}^{*} \widehat{\psi}_{s}}
$$

Then the restriction on $\Delta \xi$ is that $S(\Delta \xi)$ should not couple to the outgoing modes which have appeared by the time moment $t_{p}$. Otherwise the entanglement structure will be spoiled and the oblivion step (78) will become invalid. This restricts the shifts to

$$
\Delta \xi_{r}= \begin{cases}\text { arbitrary } & \text { for } r \geq p \\ \text { const } \times M_{p r} & \text { for } r<p\end{cases}
$$

Apart from this, there is no restrictions on the choice of importance sampling technique.

In this work we derive the importance sampling technique from the evolution of the probability distribution $Q\left(\xi, t_{p}\right)$ of the classical noise trajectories $\xi$ at a time $t_{p}$. At $t_{0}=0$ this distribution coincides with the probability distribution of the vacuum fluctuations

$$
Q(\xi, 0) \propto \exp \left(-\sum_{r s=0}^{\infty} \xi_{r}^{*}\left[M^{-1}\right]_{r s} \xi_{s}\right) .
$$

However at later time moments it becomes weighted:

$$
Q\left(\xi, t_{p}\right)=\left\|_{\mathrm{t}}\left\langle 0 \mid \Phi\left(\xi, t_{p}\right)\right\rangle_{\mathrm{st}}\right\|^{2} Q(\xi, 0),
$$

which follows from the partial trace relation eq. (72). This probability distribution is properly normalized in the sense that

$$
\overline{\left[\frac{Q\left(\xi, t_{p}\right)}{Q(\xi, 0)}\right]_{\xi}}=1
$$

which follows from $(72)$ and $\operatorname{Tr} \widehat{\rho}_{\mathrm{s}}\left(t_{p}\right)=1$. The importance sampling is required when the ratio on the left of eq. (D5) starts to fluctuate violently.

Let us find the master equation for $Q\left(\xi, t_{p}\right)$ [27]. From the tape model Hamiltonian (27) we find:

$$
\begin{aligned}
& \partial_{t} Q\left(\xi, t_{p}\right) \\
& =-i \operatorname{Tr}_{\mathrm{s}}\left\{|\xi\rangle_{\mathrm{t} \mathrm{t}}\langle\xi|\left[\widehat{H}_{\mathrm{st}}\left(\tau_{p}\right)\left|\Phi\left(t_{p}\right)\right\rangle_{\mathrm{st} \mathrm{st}}\left\langle\Phi\left(t_{p}\right)\right|-\mathrm{c.c} .\right]\right\} \\
& =-i \operatorname{Tr}_{\mathrm{s}}\left\{\left[|\xi\rangle_{\mathrm{t} \mathrm{t}}\langle\xi|\left(\widehat{s} \widehat{\psi}_{p}^{\dagger}+\widehat{s}^{\dagger} \sum_{r=0}^{\infty} M_{p r} \widehat{\psi}_{r}\right)-\text { c.c. }\right]\right. \\
& \left.\times\left|\Phi\left(t_{p}\right)\right\rangle_{\mathrm{st} \mathrm{st}}\left\langle\Phi\left(t_{p}\right)\right|\right\}, \quad(\mathrm{D} 6)
\end{aligned}
$$

where we introduce the time-cell coherent states

$$
|\xi\rangle_{\mathrm{t}}=Q^{\frac{1}{2}}(\xi, 0) e^{\sum_{s=0}^{\infty} \xi_{s} \widehat{\psi}_{s}^{\dagger}}|0\rangle_{\mathrm{t}} .
$$

In the second line of eq. (D6) we have employed the cyclic trace property and the fact that $\widehat{H}_{\mathrm{s}}$ commutes with the time-cell coherent state projections. Observe that we extend the summation to infinity in the annihilation term on the second line of eq. (D6). This is possible since the incoming modes are in vacuum. It turns out that the equation (D6) can be reduced to the convection (drift) form

$$
\begin{aligned}
\partial_{t} Q\left(\xi, t_{p}\right)=\sum_{r} \partial_{\xi_{r}} & \left\{\mathcal{A}_{r}\left(t_{p}\right) Q\left(\xi, t_{p}\right)\right\} \\
& +\sum_{r} \partial_{\xi_{r}^{*}}\left\{\mathcal{A}_{r}^{*}\left(t_{p}\right) Q\left(\xi, t_{p}\right)\right\} .
\end{aligned}
$$

In order to demonstrate this, we employ the fact that the creation $\widehat{\psi}_{s}^{\dagger}$ and annihilation $\widehat{\psi}_{s}$ operators act as differential operators on the coherent state projections:

$$
\begin{gathered}
|\xi\rangle_{\mathrm{t} \mathrm{t}}\left\langle\xi\left|\widehat{\psi}_{p}^{\dagger}=\xi_{p}^{*}\right| \xi\right\rangle_{\mathrm{t} \mathrm{t}}\langle\xi| \\
\widehat{\psi}_{p}|\xi\rangle_{\mathrm{t} \mathrm{t}}\left\langle\xi\left|=\xi_{p}\right| \xi\right\rangle_{\mathrm{t} \mathrm{t}}\langle\xi| \\
|\xi\rangle_{\mathrm{t} \mathrm{t}}\left\langle\xi\left|\sum_{r=0}^{\infty} M_{p r} \widehat{\psi}_{r}=\left\{\sum_{r=0}^{\infty} M_{p r} \partial_{\xi_{r}^{*}}+\xi_{p}\right\}\right| \xi\right\rangle_{\mathrm{t} \mathrm{t}}\langle\xi|
\end{gathered}
$$

$$
\sum_{r=0}^{\infty} M_{p r}^{*} \widehat{\psi}_{r}^{\dagger}|\xi\rangle_{\mathrm{t} \mathrm{t}}\left\langle\xi\left|=\left\{\sum_{r=0}^{\infty} M_{p r}^{*} \partial_{\xi_{r}}+\xi_{p}^{*}\right\}\right| \xi\right\rangle_{\mathrm{t} \mathrm{t}}\langle\xi| .
$$


Substituting these into the eq. (D6), we find

$$
\mathcal{A}_{r}\left(t_{p}\right)=i \bar{s}\left(t_{p} ; \xi\right) \sum_{r=0}^{\infty} M_{p r}^{*}
$$

where the conditional average $\bar{s}\left(t_{p} ; \xi\right)$ of the coupling operator $\widehat{s}$ is defined as

$$
\bar{s}\left(t_{p} ; \xi\right)=\frac{\operatorname{Tr}\left\{\widehat{s} \times_{\mathrm{t}}\left\langle 0 \mid \Phi\left(\xi, t_{p}\right)\right\rangle_{\mathrm{st} \mathrm{st}}\left\langle\Phi\left(\xi, t_{p}\right) \mid 0\right\rangle_{\mathrm{t}}\right\}}{\left\|_{\mathrm{t}}\left\langle 0 \mid \Phi\left(\xi, t_{p}\right)\right\rangle_{\mathrm{st}}\right\|^{2}}
$$

Switching to the frame of incoming/relevant/outgoing modes and employing the relevant wavefunction, we reexpress this equation as

$$
\bar{s}\left(t_{p} ; \xi\right)=\frac{\operatorname{Tr}\left\{\widehat{s} \times_{\mathrm{rel}}\left\langle 0 \mid \Phi\left(\xi, t_{p}\right)\right\rangle_{\mathrm{rel} \mathrm{rel}}\left\langle\Phi\left(\xi, t_{p}\right) \mid 0\right\rangle_{\mathrm{rel}}\right\}}{\left\|_{\mathrm{rel}}\left\langle 0 \mid \Phi\left(\xi, t_{p}\right)\right\rangle_{\mathrm{rel}}\right\|^{2}},
$$

which is the eq. (83). The velocity (D13) means that the noise trajectory $\xi$ is shifted with time as

$$
\xi\left(\tau ; t_{p}\right)=\xi(\tau)-i \int_{0}^{t_{p}} d t \bar{s}(t ; \xi) M^{*}(t-\tau) .
$$

Observe that here we switch back and forth between the continuous and the discrete-time relations. This is permissible because we have chosen the discrete propagator in sec. V B so that it converges to the continous evolution with the global error $O\left(d t^{2}\right)$.

The importance sampling procedure is to shift the noise $\xi$ continuously in time according to the relation
(D16). As we have mentioned in eq. (D1), the infinitesimal shift of noise

$$
\xi_{r}\left(t_{p+1}\right)=\xi_{r}\left(t_{p}\right)-i d t M_{p r}^{*} \bar{s}\left(\tau_{p} ; \xi\right)
$$

will generate the infinitesimal Bogoliubov transform

$$
S(d \xi)=1+i d t \bar{s}^{*}\left(\tau_{p} ; \xi\right) \sum_{r=0}^{p-1} M_{p r} \widehat{\psi}_{r}
$$

which should be incorporated into the Hamiltonian. The latter for the continuously shifting noise becomes

$$
\begin{aligned}
& \widehat{H}_{\mathrm{st}}^{\prime}\left(\xi, \tau_{p}\right)=\widehat{H}_{\mathrm{s}}+\widehat{s}\left\{\widehat{\psi}_{p}^{\dagger}+\xi_{p}^{*}+f_{p}^{*}\right\} \\
& +\left(\widehat{s}^{\dagger}-\bar{s}^{*}\left(\tau_{p} ; \xi\right)\right)\left\{M(0) \widehat{\psi}_{p}+\sum_{i=1}^{\min (p, m)} M_{i}(p) \widehat{\phi}_{i}\right\}
\end{aligned}
$$

which is the eq. (86). Here $\xi_{p}^{*}$ are sampled from the vacuum probability distribution, and the self-consistent displacement is

$$
f_{p}=-i \int_{0}^{t_{p}} d t \bar{s}(t ; \xi) M\left(\tau_{p}-t\right)
$$

The consistent midpoint discretization of $f_{p}$ leads to equation (87). The Hamiltonian $\widehat{H}_{\text {st }}^{\prime}\left(\xi, \tau_{p}\right)$ should be employed in the entangling step eq. (75). The rest of $\mathrm{RG}$ procedure is the same except that the average of any system observable $\widehat{o}$ should be computed as

$$
\left\langle\widehat{o}\left(t_{p}\right)\right\rangle=\overline{\left[\frac{\operatorname{Tr}\left\{\widehat{o} \times{ }_{\mathrm{rel}}\left\langle 0 \mid \Phi\left(\xi, t_{p}\right)\right\rangle_{\mathrm{rel} \mathrm{rel}}\left\langle\Phi\left(\xi, t_{p}\right) \mid 0\right\rangle_{\mathrm{rel}}\right\}}{\left\|_{\mathrm{rel}}\left\langle 0 \mid \Phi\left(\xi, t_{p}\right)\right\rangle_{\mathrm{rel}}\right\|^{2}}\right]_{\xi}} .
$$

The reason is that now we directly sample the probability distribution (D4), hence the denominator.
[1] R. P. Feynman, A. R. Hibbs, and D. F. Styer, Quantum Mechanics and Path Integrals: Emended Edition (Dover Publications, 2010).

[2] P. A. M. Dirac, The Principles of Quantum Mechanics (Clarendon Press, 1982).

[3] D. Poulin, A. Quarry, R. Somma, and F. Verstraete, Quantum simulation of time-dependent hamiltonians and the convenient illusion of hilbert space, Phys. Rev. Lett. 106, 170501 (2011).

[4] F. G. Brandao, W. Chemissany, N. Hunter-Jones, R. Kueng, and J. Preskill, Models of quantum complexity growth (2019), arXiv:1912.04297 [hep-th].

[5] S.-H. Lin, R. Dilip, A. G. Green, A. Smith, and F. Pollmann, Real- and imaginary-time evolution with compressed quantum circuits, PRX Quantum 2, 010342 (2021).
[6] Y. Zhou, E. M. Stoundenmire, and X. Waintal, What limits the simulation of quantum computers?, Phys. Rev. X 10, 041038 (2020).

[7] D. S. Franca and R. Garcia-Patron, Limitations of optimization algorithms on noisy quantum devices (2020), arXiv:2009.05532 [quant-ph].

[8] G. Vidal, Entanglement renormalizatiom, Phys. Rev. Lett. 99, 220405 (2007).

[9] G. Vidal, Class of quantum many-body states that can be efficiently simulated, Phys. Rev. Lett. 101, 110501 (2008).

[10] K. V. Acoleyen, A. Hallam, M. Bal, M. Hauru, J. Haegeman, and F. Verstraete, Entanglement compression in scale space: From the multiscale entanglement renormalization ansatz to matrix product operators, Phys. Rev. B 102, 165131 (2020). 
[11] J. Haegeman, T. J. Osborne, H. Verschelde, and F. Verstraete, Entanglement renormalization for quantum fields in real space, Phys. Rev. Lett. 110, 100402 (2013).

[12] S. R. White, Density matrix formulation for quantum renormalization groups, Phys. Rev. Lett. 69, 2863 (1992).

[13] U. Schollwock, The density-matrix renormalization group in the age of matrix product states, Ann. Phys. 326, 96 (2011).

[14] I. d. Vega and D. Alonso, Dynamics of non-markovian open quantum systems, Rev. Mod. Phys. 89, 015001 (2017).

[15] T. Brandes, Feedback control of quantum transport, Phys. Rev. Lett. 105, 060602 (2010).

[16] G. Kiesslich, C. Emary, G. Schaller, and T. Brandes, Reverse quantum state engineering using electronic feedback loops, New J. Phys. 14, 123036 (2012).

[17] J. Gough, Feedback network models for quantum transport, Phys. Rev. E 90, 062109 (2014).

[18] T. Brandes and C. Emary, Feedback control of waiting times, Phys. Rev. E 93, 042103 (2016).

[19] J. Y. Luo, J. Jin, S.-K. Wang, J. Hu, Y. Huang, and X.L. He, Unraveling of a detailed-balance-preserved quantum master equation and continuous feedback control of a measured qubit, Phys. Rev. B 93, 125122 (2016).

[20] T. Wagner, P. Strasberg, J. C. Bayer, E. P. Rugeramigabo, T. Brandes, and R. J. Haug, Strong suppression of shot noise in a feedback-controlled single-electron transistor, Nat. Nanotechnol. 12, 218 (2017).

[21] J. Kondo, Diffusion of light interstitials in metals, Physica B + C 125, 279 (1984).

[22] K. G. Wilson, The renormalization group: Critical phenomena and the kondo problem, Rev. Mod. Phys. 47, 773 (1975).

[23] L. Bulla and M. Vojta, Quantum phase transitions in models of magnetic impurities, in Concepts in Electron Correlations. NATO Science Series (Series II: Mathematics, Physics and Chemistry), Vol. 110, edited by A. C. Hewson and V. Zlatic (Springer, Dordrecht, 2003) pp. 209-217.

[24] R. Bulla, T. A. Costi, and T. Pruschke, Numerical renormalization group method for quantum impurity systems, Rev. Mod. Phys. 80, 395 (2008).

[25] C. Lazarou, K. Luoma, S. Maniscalco, J. Piilo, and B. M. Garraway, Entanglement trapping in a nonstationary structured reservoir, Phys. Rev. A 86, 012331 (2012).

[26] M. M. Rams and M. Zwolak, Breaking the entanglement barrier: Tensor network simulation of quantum transport, Phys. Rev. Lett. 127, 137701 (2020).

[27] E. A. Polyakov and A. N. Rubtsov, Dressed quantum trajectories: novel approach to the non-markovian dynamics of open quantum systems on a wide time scale, New. J. Phys. 21, 063004 (2019).

[28] A. Strathearn, P. Kirton, D. Kilda, J. Keeling, and B. W. Lovett, Efficient non-markovian quantum dynamics using time-evolving matrix product operators, Nat. Commun. 9, 3322 (2018).

[29] J. Eisert, M. Cramer, and M. B. Plenio, Colloquium: Area laws for the entanglement entropy, Rev. Mod. Phys. 82, 277 (2010).

[30] H.-P. Breuer and F. Petruccione, The Theory of Open Quantum Systems (Oxford University Press, Oxford, 2007).

[31] I. Percival, Quantum State Diffusion (Cambridge University Press, Cambridge, 1999).
[32] H. M. Wiseman, Quantum trajectories and quantum measurement theory, Quantum Semiclass. Opt. 8, 205 (1996).

[33] H. M. Wiseman and G. J. Milburn, Quantum theory of field-quadrature measurements, Phys. Rev. A 47, 642 (1993).

[34] A. J. Daley, Quantum trajectories and open many-body quantum systems, Adv. in Phys. 63, 77 (2014).

[35] D. Segal, A. J. Millis, and D. R. Reichman, Numerically exact path-integral simulation of nonequilibrium quantum transport and dissipation, Phys. Rev. B 82, 205323 (2010).

[36] N. Makri, Numerical path integral techniques for long time dynamics of quantum dissipative systems, Journal of Mathematical Physics 36, 2430 (1995), https://doi.org/10.1063/1.531046.

[37] N. Makri and D. E. Makarov, Tensor propagator for iterative quantum time evolution of reduced density matrices. i. theory, The Journal of Chemical Physics 102, 4600 (1995), https://doi.org/10.1063/1.469508.

[38] N. Makri, E. Sim, D. E. Makarov, and M. Topaler, Long-time quantum simulation of the primary charge separation in bacterial photosynthesis, Proceedings of the National Academy of Sciences 93, 3926 (1996), http://www.pnas.org/content/93/9/3926.full.pdf.

[39] D. E. Makarov and N. Makri, Path integrals for dissipative systems by tensor multiplication. condensed phase quantum dynamics for arbitrarily long time, Chemical Physics Letters 221, 482 (1994).

[40] M. Richter and B. P. Fingerhut, Coarse-grained representation of the quasi-adiabatic propagator path integral for the treatment of non-markovian long-time bath memory, J. Chem. Phys. 146, 214101 (2017).

[41] M. P. Woods, R. Groux, A. W. Chin, S. F. Huelga, and M. B. Plenio, Mappings of open quantum systems onto chain representations and markovian embeddings, J. Math. Phys. 55, 032101 (2014).

[42] L. Mazzola, S. Maniscalco, J. Piilo, K.-A. Suominen, and B. M. Garraway, Pseudomodes as an effective description of memory: Non-markovian dynamics of two-state systems in structured reservoirs, Phys. Rev. A 80, 012104 (2009).

[43] N. Lambert, S. Ahmed, M. Cirio, and F. Nori, Modelling the ultra-strongly coupled spin-boson model with unphysical modes, Nat. Commun. 10, 3721 (2019).

[44] D. Tamascelli, A. Smirne, S. F. Huelga, and M. B. Plenio, Nonperturbative treatment of non-markovian dynamics of open quantum systems, Phys. Rev. Lett. 120, 030402 (2018).

[45] M. R. Jorgensen and F. A. Pollock, Exploiting the causal tensor network structure of quantum processes to efficiently simulate non-markovian path integrals, Phys. Rev. Lett. 123, 240602 (2019).

[46] I. A. Luchnikov, S. V. Vintskevich, H. Querdane, and S. N. Filippov, Simulation complexity of open quantum dynamics: Connection with tensor networks, Phys. Rev. Lett. 122, 160401 (2019).

[47] A. D. Somoza, O. Marty, J. Lin, S. F. Huelga, and M. B. Plenio, Dissipation-assisted matrix product factorization, Phys. Rev. Lett. 123, 100502 (2019).

[48] W. Zhu, Z. Huang, Y.-C. He, and X. Wen, Entanglement hamiltonian of many-body dynamics in strongly correlated systems, Phys. Rev. Lett. 2020, 124 (2020).

[49] L. Diosi, N. Gisin, and W. T. Strunz, Non-markovian 
quantum state diffusion, Phys.l Rev. A 58, 1699 (1998).

[50] L. Diosi and W. T. Strunz, The non-markovian stochastic schrijdinger equation for open systems, Phys. Lett. A 235, 569 (1997).

[51] Y. Zhou and J. Shao, Solving the spin-boson model of strong dissipation with flexible random-deterministic scheme, J. Chem. Phys. 128, 034106 (2008).

[52] Y.-A. Yan and J. Shao, Stochastic description of quantum brownian dynamics, Front. Phys. 11, 110309 (2016).

[53] J. Shao, Decoupling quantum dissipation via stochastic fields, J. Chem. Phys. 120, 5053 (2004).

[54] L. Han, V. Chemyak, Y.-A. Yan, X. Zheng, and Y. Yan, Stochastic representation of non-markovian fermionic quantum dissipation, Phys. Rev. Lett. 123, 050601 (2019).

[55] D. Suess, A. Eisfeld, and W. T. Strunz, Hierarchy of stochastic pure states for open quantum system dynamics, Phys. Rev. Lett. 113, 150403 (2014).

[56] R. Hartmann and W. T. Strunz, Exact open quantum system dynamics using the hierarchy of pure states (hops), J. Chem. Theor. Comput. 13, 5834 (2017).

[57] J. M. Moix and J. Cao, A hybrid stochastic hierarchy equations of motion approach to treat the low temperature dynamics of non-markovian open quantum systems, J. Chem. Phys 139, 134106 (2013).

[58] J. StrÃCEmpfer and K. Schulten, Open quantum dynamics calculations with the hierarchy equations of motion on parallel computers, Journal of Chemical Theory and Computation 8, 2808 (2012), pMID: 23105920, http://dx.doi.org/10.1021/ct3003833.

[59] A. Ishizaki and G. R. Fleming, Unified treatment of quantum coherent and incoherent hopping dynamics in electronic energy transfer: Reduced hierarchy equation approach, The Journal of Chemical Physics 130, 234111 (2009), https://doi.org/10.1063/1.3155372.

[60] C. Duan, Z. Tang, J. Cao, and J. Wu, Zero-temperature localization in a sub-ohmic spin-boson model investigated by an extended hierarchy equation of motion, Phys. Rev. B 95, 214308 (2017).

[61] H. Rahman and U. Kleinekathofer, Chebyshev hierarchical equations of motion for systems with arbitrary spectral densities and temperatures, J. Chem. Phys. 150, 244104 (2019).

[62] E. A. Polyakov and A. N. Rubtsov, Stochastic wavefunction simulation of irreversible emission processes for open quantum systems in a non-markovian environment, AIP Conf. Proc. 1936, 020028 (2018).

[63] E. A. Polyakov and A. N. Rubtsov, Information loss pathways in a nuremically exact simulation of a nonmarkovian open quantum system (2018), to be published.

[64] L. Diosi, Non-markovian open quantum systems: Inputoutput fields, memory, and monitoring, Phys. Rev. A 85, 034101 (2012).

[65] A. Atland and B. D. Simons, Condensed Matter Field Theory (Cambridge University Press, New York, 2010).

[66] M. B. Plenio and P. L. Knight, The quantum-jump approach to dissipative dynamics in quantum optics, Rev. Mod. Phys. 70, 101 (1998).

[67] Y. Aharonov and L. Vaidman, The two-state vector formalism: An updated review, in Time in Quantum Mechanics, Lecture Notes in Physics, Vol. 734, edited by J. Muga, R. S. Mayato, and I. Egusquiza (Springer, Berlin, 2008) Chap. 13, pp. 399-447.

[68] Quantum Theory: A Two-Time Success Story (Springer,
Milano, 2014).

[69] Y. Aharonov, E. Cohen, and T. Landsberger, The twotime interpretation and macroscopic time-reversibility, Entropy 19, 111 (2017).

[70] C. W. Gardiner and P. Zoller, Quantum Noise, 3rd ed. (Springer-Verlag Berlin Heidelberg, 2004).

[71] M. B. Plenio and S. Virmani, An introduction to the entanglement measures, Quantum Info. Comput. 7, 1 (2007).

[72] A. W. Chin, A. Rivas, S. F. Huelga, and M. B. Plenio, Exact mapping between system-reservoir quantum models and semi-infinite discrete chains using orthogonal polynomials, J. Math. Phys. 51, 092109 (2010).

[73] H.-P. Breuer, E.-M. Laine, and J. Piilo, Measure for the degree of non-markoviani behaviour of quantum processes in open systems, Phys. Rev. Lett. 103, 210401 (2009).

[74] B. Bylicka, M. Johansson, and A. Acin, Constructive method for detecting the information backflow of non-markovian dynamics, Phys. Rev. Lett 118, 120501 (2017).

[75] F. Buscemi and N. Datta, Equivalence between divisibility and monotonic decrease of information in classical and quantum stochastic processes, Phys. Rev. A 93, 012101 (2016).

[76] M. B. Ruskai, Beyond strong subadditivity? improved bounds on the contraction of generalized relative entropy, Rev. Math. Phys. 6, 1147 (2009).

[77] M. Popovic, M. T. Mitchison, A. Strathearn, B. W. Lovett, J. Goold, and P. R. Eastham, Quantum heat statistics with time-evolving matrix product operators, PRX Quantum 2, 020338 (2021).

[78] A. Strathearn, P. Kirton, D. Kilda, J. Keeling, and B. W. Lovett, Efficient non-markovian quantum dynamics using time-evolving matrix product operators, https://doi. org/10.5281/zenodo.1322407 (2018).

[79] T. Ali, A. Bhattacharyya, S. S. Haque, E. H. Kim, and N. Moynihan, Time evolution of complexity: a critique of three methods, JHEP 2019, 87.

[80] A. Bhattacharyya, P. Nandy, and A. Sinha, Renormalized circuit complexity, Phys. Rev. Lett. 124, 101602 (2020).

[81] N. Megier, W. N. Strunz, and K. Luoma, Continuous quantum measurement for general gaussian unravelings can exist (2020), arXiv:1912.08662 [quant-ph].

[82] S. L. Adler and A. Bassi, Collapse models with non-white noises, J. Phys. A: Math. Theor. 40, 15083 (2007).

[83] M. Carlesso, L. Ferialdi, and A. Bassi, Colored collapse models from the non-interferometric perspective, EPJ D 72, 159 (2018).

[84] W. T. Strunz, L. Diosi, N. Gisin, and T. Yu, Quantum trajectories for brownian motion, Phys. Rev. Lett. 83, 4909 (1999).

[85] W. T. Strunz, L. Diosi, and N. Gisin, Open system dynamics with non-markovian quantum trajectories, Phys. Rev. A 82, 1801 (1999).

[86] J. Gambetta, T. Askerud, and H. M. Wiseman, Jumplike unravelings for non-markovian open quantum systems, Phys. Rev. A 69, 052104 (2004).

[87] H.-P. Breuer, Genuine quantum trajectories for nonmarkovian processes, Phys. Rev. A 70, 012106 (2004).

[88] K. Luoma, K.-A. Suominen, and J. Piilo, Connecting two jumplike unravelings for non-markovian open quantum systems, Phys. Rev. A 84, 032113 (2011).

[89] D. Durr, S. Goldstein, R. Tumulka, and N. Zanghi, 
Bohmian mechanics and quantum field theory, Phys. Rev. Lett. 93, 090402 (2004). 